Review Article

\title{
Natural Phytochemicals as Novel Therapeutic Strategies to Prevent and Treat Parkinson's Disease: Current Knowledge and Future Perspectives
}

\author{
Rengasamy Balakrishnan $\mathbb{D}^{1,2}$ Shofiul Azam, ${ }^{1}$ Duk-Yeon Cho, ${ }^{1}$ In Su-Kim, ${ }^{2}$ \\ and Dong-Kug Choi ${ }^{1,2}$ \\ ${ }^{1}$ Department of Applied Life Science, Graduate School, BK21 Program, Konkuk University, Chungju 27478, Republic of Korea \\ ${ }^{2}$ Department of Biotechnology, College of Biomedical and Health Science, Research Institute of Inflammatory Disease (RID), \\ Konkuk University, Chungju 27478, Republic of Korea
}

Correspondence should be addressed to Dong-Kug Choi; choidk@kku.ac.kr

Received 16 December 2020; Revised 14 April 2021; Accepted 26 April 2021; Published 25 May 2021

Academic Editor: Erica Buoso

Copyright (c) 2021 Rengasamy Balakrishnan et al. This is an open access article distributed under the Creative Commons Attribution License, which permits unrestricted use, distribution, and reproduction in any medium, provided the original work is properly cited.

\begin{abstract}
Parkinson's disease (PD) is the second-most common neurodegenerative chronic disease affecting both cognitive performance and motor functions in aged people. Yet despite the prevalence of this disease, the current therapeutic options for the management of PD can only alleviate motor symptoms. Research has explored novel substances for naturally derived antioxidant phytochemicals with potential therapeutic benefits for PD patients through their neuroprotective mechanism, targeting oxidative stress, neuroinflammation, abnormal protein accumulation, mitochondrial dysfunction, endoplasmic reticulum stress, neurotrophic factor deficit, and apoptosis. The aim of the present study is to perform a comprehensive evaluation of naturally derived antioxidant phytochemicals with neuroprotective or therapeutic activities in PD, focusing on their neuropharmacological mechanisms, including modulation of antioxidant and anti-inflammatory activity, growth factor induction, neurotransmitter activity, direct regulation of mitochondrial apoptotic machinery, prevention of protein aggregation via modulation of protein folding, modification of cell signaling pathways, enhanced systemic immunity, autophagy, and proteasome activity. In addition, we provide data showing the relationship between nuclear factor E2-related factor 2 (Nrf2) and PD is supported by studies demonstrating that antiparkinsonian phytochemicals can activate the Nrf2/antioxidant response element (ARE) signaling pathway and Nrf2-dependent protein expression, preventing cellular oxidative damage and PD. Furthermore, we explore several experimental models that evaluated the potential neuroprotective efficacy of antioxidant phytochemical derivatives for their inhibitory effects on oxidative stress and neuroinflammation in the brain. Finally, we highlight recent developments in the nanodelivery of antioxidant phytochemicals and its neuroprotective application against pathological conditions associated with oxidative stress. In conclusion, naturally derived antioxidant phytochemicals can be considered as future pharmaceutical drug candidates to potentially alleviate symptoms or slow the progression of PD. However, further well-designed clinical studies are required to evaluate the protective and therapeutic benefits of phytochemicals as promising drugs in the management of PD.
\end{abstract}

\section{Introduction}

Parkinson's disease (PD) is a common progressive chronic neurodegenerative movement disorder that increases with age. PD prevalence is 315 per 100000 persons of all ages in the Western world; this prevalence is expected to double by the year 2030, increasing mortality, morbidity, and socioeco- nomic burden worldwide [1]. The clinical symptoms commonly associated with PD disorder include bradykinesia, resting tremor, postural instability, rigidity, depression, and anxiety [2]. The important hallmarks of PD are progressive loss or damage of dopaminergic neurons in the substantia nigra pars compacta (SNpc) and dopamine (DA) depletion in the striatum (ST), which is associated with the motor 
impairments of PD [3]. In addition to the neuropathological process affecting dopaminergic and nondopaminergic systems, other pathological processes are also seen in Alzheimer's disease (AD) affecting cholinergic dysfunction and serotonergic, glutamatergic, and noradrenergic pathways associated with dopaminergic neuronal death and/or DA system dysfunction. People with $\mathrm{PD}$ also experience nonmotor symptoms such as sleep disturbance, cognitive changes, autonomic dysfunction, altered mood, depression, fatigue, and pain $[4,5]$. PD is described as a synucleinopathy, as accumulation of misfolded $\alpha$-synuclein becomes a central feature of Lewy bodies, which are an important pathological hallmark of PD. Moreover, $\alpha$-synuclein appears to be linked to both sporadic and familial forms of the disease and carries unique importance in the etiology of PD [6]. Interestingly, $\alpha$-synuclein accumulation has been broadly linked to several neurotoxin pathways, including posttranslational modifications, neuroinflammation, oxidative stress, mitochondrial dysfunction, altered mitochondrial morphology, synaptic dysfunction, phospholipids, induced endoplasmic reticulum (ER) stress, and metal ions [7]. The age-related failure of antioxidant defense system and overproduction of ROS exacerbate oxidative stress in the brain; these events may play a role of misfolded $\alpha$-synuclein initiating aging process in PD $[8,9]$.

Currently, levodopa (L-dopa) is the most effective therapy for the early-stage motor symptoms of PD, but it is not considered a cure for PD [10]. Bradykinesia and rigidity respond best, whereas tremor may be only slightly reduced. Problems with balance and other symptoms may not be alleviated at all. However, L-dopa is not effective in relieving neuronal loss, nonmotor symptoms, or Lewy pathology. Over time, patients require higher doses of L-dopa, which are associated with increased side effects such as dyskinesia [11]. Anticholinergic drugs may help control rigidity and tremor in approximately $50 \%$ of cases, and the antiviral agent amantadine also seems to diminish motor symptoms [3]. Deep brain stimulation (DBS) and DA-based medications are also used to treat various neurologic motor symptoms with disease progression [12]. Hence, it is critical to develop new therapeutic approaches to prevent neuronal loss and nonmotor symptoms and to prevent the accumulation of $\alpha$ synuclein aggregation or Lewy pathology in the brain. Moreover, only symptomatic treatment options are available for $\mathrm{PD}$; none slow or prevent progressive neuronal loss in the dopaminergic system $[13,14]$. Herbal preparation and phytochemicals isolated from plant food have been proposed as "herbal medicine" for the treatment of PD [15]. Myriad phytochemicals from nature have been documented as potential molecules, drug leads, and phytochemical formulations in treating several inflammatory disorders [16, 17]. Likewise, extensive pharmacological reports have demonstrated the effectiveness of phytochemicals in treating dementia, depression, and neurodegenerative disorders (NDDs) [18]. Biologically active phytochemicals produced in plants are of clinical importance as primary and secondary metabolites for the antioxidant defense mechanism against various stress-related disorders and other pathogenic conditions [19]. The therapeutic and beneficial effects of these phytochemicals provide nutrition for normal living cells, fight disease-causing agents, strengthen the immune system, and act as antioxidants [20]. Plant products and their bioactive phytochemicals can efficiently scavenge oxygen free radicals and boost the cellular antioxidant defense system and related molecules, thereby protecting cells from oxidative damage $[20,21]$. Several findings indicate that these antioxidant phytochemicals have confirmed neuritogenic potential, reconstructing synaptic connectivity by restoring the loss of neuronal processes [22-25]. In fact, various preclinical reports have described a number of natural pharmacological candidates that can coactivate the antioxidant defense system and neurotrophic factor-mediated cell survival systems [26-31], suggesting that these phytochemicals have therapeutic potential for the treatment of oxidative stress-mediated NDDs, especially PD.

Thus, therapeutic approaches targeting oxidative stress, $\alpha$-synuclein accumulation, neuroinflammation, and mitochondrial dysfunction may hold great promise as a cure for PD. Numerous antioxidant phytochemicals have displayed potentially neuroprotective properties by targeting several mechanisms beyond those mentioned above. Phytochemicals are biologically active compounds that usually correspond to the secondary metabolites present in plants like alkaloids, flavonoids, and terpenoids [32]. Many epidemiological studies have suggested a proportional relationship between consumption of a diet rich in antioxidant phytochemicals and improved health outcomes, including reduced risk for $\mathrm{AD}$, PD, and other NDDs [33-35]. Other epidemiological studies have associated the consumption of various food groups and beverages such as fruits, vegetables, tea, and coffee with reduced risk of development of PD [36, 37]. Recent research on the dietary intake of phenolic phytochemicals has been presented in several European countries, with results showing that the average intake is $820 \mathrm{mg} /$ day in Spain, $1193 \mathrm{mg} /$ day in France, and $1756.5 \mathrm{mg} /$ day in Poland. The main dietary sources of the total polyphenols in Spain and France are fruits and nonalcoholic beverages (principally coffee and tea). In Spain, fruits accounted for $44 \%$ and nonalcoholic beverages for $23 \%$ of total polyphenol intake, whereas in France fruit accounted for only $17 \%$ and nonalcoholic beverages for $55 \%$. Considered individually, the main source of total dietary polyphenols is food with $18 \%$ and $44 \%$ of contribution in Spain and France, respectively. In Spain, olives and olive oils are also important sources of polyphenols, accounting for $11 \%$ of the total polyphenol intake. Nonalcoholic beverages were the main food contributors to polyphenol intake in Poland and accounted for fully $67 \%$ of the total polyphenol intake due to high consumption of coffee and tea. The third main contributor to total polyphenol intake is chocolate, whereas fruits accounted for a lower percentage of intake [38-40].

In the present study, we describe the phytochemicals present in dietary sources, using chrysin, vanillin, ferulic acid (FA), thymoquinone (TQ), ellagic acid (EA), caffeic acid (CA), epigallocatechin-3-gallate (EGCG), theaflavin (TF), and other plant-derived antioxidant phytochemicals (asiatic acid (AA) and $\alpha$ - and $\beta$-asarone) as examples and discuss their beneficial neuroprotective effects and relevance to potential treatment strategies of PD. Importantly, phytochemicals 
have thus far been investigated primarily in both cellular and rodent experimental studies for their potential benefits in brain metabolism; these studies have provided some encouraging results indicating antioxidant, anti-inflammatory, and cognitive enhancing effects of these phytochemicals coupled with a wide range of tolerability [41, 42]. Additionally, phytochemicals also have been confirmed to reduce mitochondrial dysfunction and inhibit formation of $\alpha$-synuclein accumulation-induced oxidative stress and inflammatory responses $[43,44]$. Several studies have also provided evidence that the antioxidant activity of some phytochemicals can activate nuclear factor E2-related factor 2 (Nrf2)/antioxidant response element (ARE) signaling pathways. Furthermore, phytochemicals contribute to the activation of the phosphatidylinositol 3-kinase/protein kinase B (PI3K/Akt) and extracellular signal-regulated kinase (ERK) pathways and inhibit nuclear factor kappa B (NF- $\kappa$ B) pathways $[22,45]$. Similarly, a number of studies have suggested that phytochemicals confer neuroprotection in experimental parkinsonism by reducing oxidative stress and mitochondrial dysfunction and fostering degradation of $\alpha$-synuclein toxic species through activation of autophagy [46-48]. This study provides information about the neuroprotective properties and mechanisms of action of recently discovered naturally derived phytochemicals that target oxidative stress and neurodegeneration through cellular- and molecular-level changes in the progression of $\mathrm{PD}$. In addition, we explore Nrf2/ARE and autophagy signaling-related pharmacological mechanisms. Moreover, we highlight some potentially neuroprotective active derivatives of antioxidant phytochemicals and phytochemicalbased nanodelivery systems that fight pathological conditions associated with aging-related oxidative stress. The sources and chemical structures of phytochemicals are presented in Figure 1.

\section{Role of Oxidative Stress in PD}

Oxygen is a crucial molecule that may produce free radicals during metabolic reactions. These free radicals constitute an important fundamental molecule in any biochemical and/or biological reduction reaction [49]. However, these free radicals are highly unstable and easily cross biomolecules like nucleic acid, lipids, cellular membranes, and proteins [50]. In addition, recent investigations have observed novel developments in the biology of free radicals and their impact on brain health and the development of various pathological conditions, since the brain uses a disproportionately large volume of oxygen compared to other organs, making it more vulnerable to free radical attacks $[51,52]$. Free radicals like reactive nitrogen species (RNS) and reactive oxygen species (ROS) are prime stimulators of oxidative stress and imbalance in the antioxidant capability of cells [53]. ROS, such as hydrogen peroxide $\left(\mathrm{H}_{2} \mathrm{O}_{2}\right)$, are key precursors of superoxide radicals $(\mathrm{O}-2)$ produced in the brain mitochondria that restrict mitochondrial movement and damage DNA, leading to impaired brain function and NDDs [54]. Considerable evidence from recent in vivo experiments has confirmed that excessive production of ROS contributes significantly to neuronal cell death and altered brain function resulting from DA metabolism, low levels of glutathione (GSH), and high levels of calcium and iron in the substantia nigra (SN) $[55,56]$. Moreover, the brain contains a high level of membrane polyunsaturated fatty acids, which, under oxidative stress conditions, leads to higher lipid peroxidation and the generation of neurotoxic products. These deficient enzymatic antioxidant systems and increased oxidative stress markers are common pathological hallmarks of PD [57]. ROS contributions to PD pathogenesis have been supported by extensive evidence (Figure 2).

Several studies have previously reported that PD tissues show an oxidant status that is broadly associated with both DA autooxidation and mitochondrial complex I dysfunction [58]. In addition, preclinical and clinical findings have revealed that brain and cerebrospinal fluid samples from PD patients contain significantly higher levels of oxidized coenzyme Q10 and the nuclear DNA oxidation biomarker 8-OHdG, establishing the existence of oxidative damage to DNA and mitochondria in PD patients [59]. Moreover, reduced mitochondrial complex I catalytic activity, downregulated mitochondrial biogenesis, and reduced mitochondrial DNA levels in the prefrontal cortex were observed contributing to excessive ROS production and oxidative stress in $\mathrm{PD}$ brains [60]. Recent findings have identified excess production of ROS and diminished mitochondrial complex I activity in SH-SY5Y neuroblastoma cells [61]. Similarly, one model using 6-hydroxydopamine- (6-OHDA-) induced rats found increased levels of 8-OHdG in the urine, serum, and $\mathrm{SN}$ of rats when compared with control groups; this finding was consistent with research involving human tissue [62]. In addition, earlier studies have demonstrated that increased ROS production causes significant and simultaneous dysregulation of several signaling pathways, such as RAS-MEKERK1/2 [63], PI3K/AKT/GSK3 $\beta$ [64], Keap1-Nrf2-ARE [65], NF- $\kappa$ B [66-68], and JAK/STAT [69].

\section{Role of Autophagy in PD}

Autophagy is the key physiological cellular catabolic process in response to cellular starvation and the degradation of damaged organelles [70]. Autophagy describes the process by which diverse cellular mechanisms encompassing nucleic acids, whole organelles, lipids, proteins, sugars, and cytoplasmic compartments are sequestered into a double-membrane budding vacuole called a phagophore, which later matures to seal in a vesicle called an autophagosome [71, 72]. Damaged or dysregulated autophagy has been linked with several pathological processes, including inflammation, cancer, lipid metabolism, and NDDs [73-76]. In fact, autophagy helps clear damaged organelles, protein aggregates, and lipid droplets, which constitute unwanted and often toxic cargo that may lead to cellular dysfunction. Importantly, damaged or dysregulated autophagy has been recognized as a critical pathogenic process, particularly in PD and other NDDs [73, 77].

In $\mathrm{PD}$, the role of autophagy is significant in neuronal quality control and brain maintenance. Recent confirmations have demonstrated that mice lacking key autophagy genes like AuTophaGy ATG5 and ATG7 show spontaneous neurodegeneration, accumulation of protein aggregates, and motor 

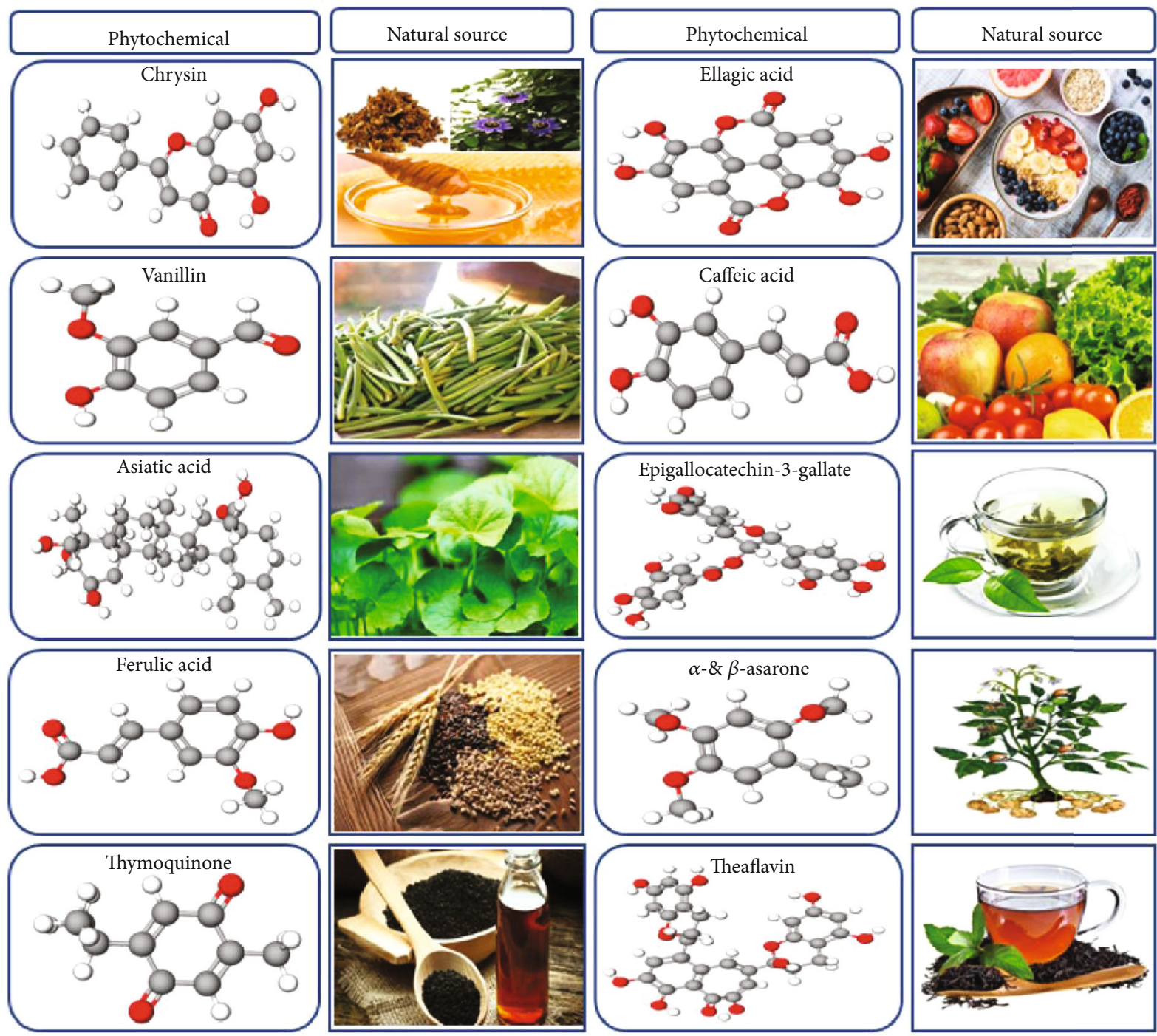

Figure 1: Structures of phytochemicals present in dietary sources (chrysin, vanillin, ferulic acid (FA), thymoquinone (TQ), ellagic acid (EA), caffeic acid (CA), epigallocatechin-3-gallate (EGCG), and theaflavin (TF)) and other plant-derived phytochemicals (asiatic acid (AA) and $\alpha$ and $\beta$-asarone), belonging to different classes of phenolics and nonphenolics. These phytochemicals have demonstrated several mechanisms of action by which they protect the brain from neurodegeneration. The structures were regenerated from http://molview.org/. Here, the different colors of different atoms in the structures represent specific molecules, where grey $=$ carbon, white $=$ hydrogen, and red $=$ oxygen .

neuron dysfunction [78, 79]. 6-OHDA, 1-methyl-4-phenylpyridinium $\left(\mathrm{MPP}^{+}\right)$, rotenone, and 1-methyl-4-phenyl-1, 2,3,6-tetrahydropyridine (MPTP) - a neurotoxin associated with mitochondrial dysfunction, ATP depletion, and accumulation of ROS such as $\mathrm{H}_{2} \mathrm{O}_{2}$, hydroxyl radicals, and superoxide-are commonly used in experimental models of PD in both in vitro and in vivo research $[80,81]$. Changes in autophagy status have been reported in 6-OHDA-induced PD models [82, 83]. Recently, it was reported that a mild increase in ROS levels could activate mucolipin 1, a key calcium-conducting channel located on the lysosome membrane, to initiate calcineurin-dependent activation of transcription factor $\mathrm{EB}$, which is identified as a master regulator of the autophagy-lysosome pathway (ALP) [84]. In turn, the transcription factor EB-mediated induction of autophagy promotes clearance of damaged mitochondria and removal of excess ROS [73]. Excessive ROS levels may cause lysosomal dysfunction and autophagic failure and lead to oxidative stress or cell death; however, oxidative stress generally mediates autophagic pathway activation [85]. Moreover, the activation levels and induction time of autophagy also play critical roles in the survival or death of cells [86].

As mentioned previously, DJ-1 has been confirmed to regulate basal autophagy and mitophagy in a manner similar to Parkin and PINK1 $[87,88]$. Recently, it has been demonstrated that DJ-1 loss-of-function mutations were first identified as generating oxidative stress in mice and Drosophila exposed to the toxicity of rotenone, paraquat (PQ), and MPTP $[89,90]$. Interestingly, DJ-1 overexpression has been shown to protect against oxidative insults. In dopaminergic cell lines, the overexpression of DJ-1 was able to protect dopaminergic cells through reduced levels of protein oxidation, 


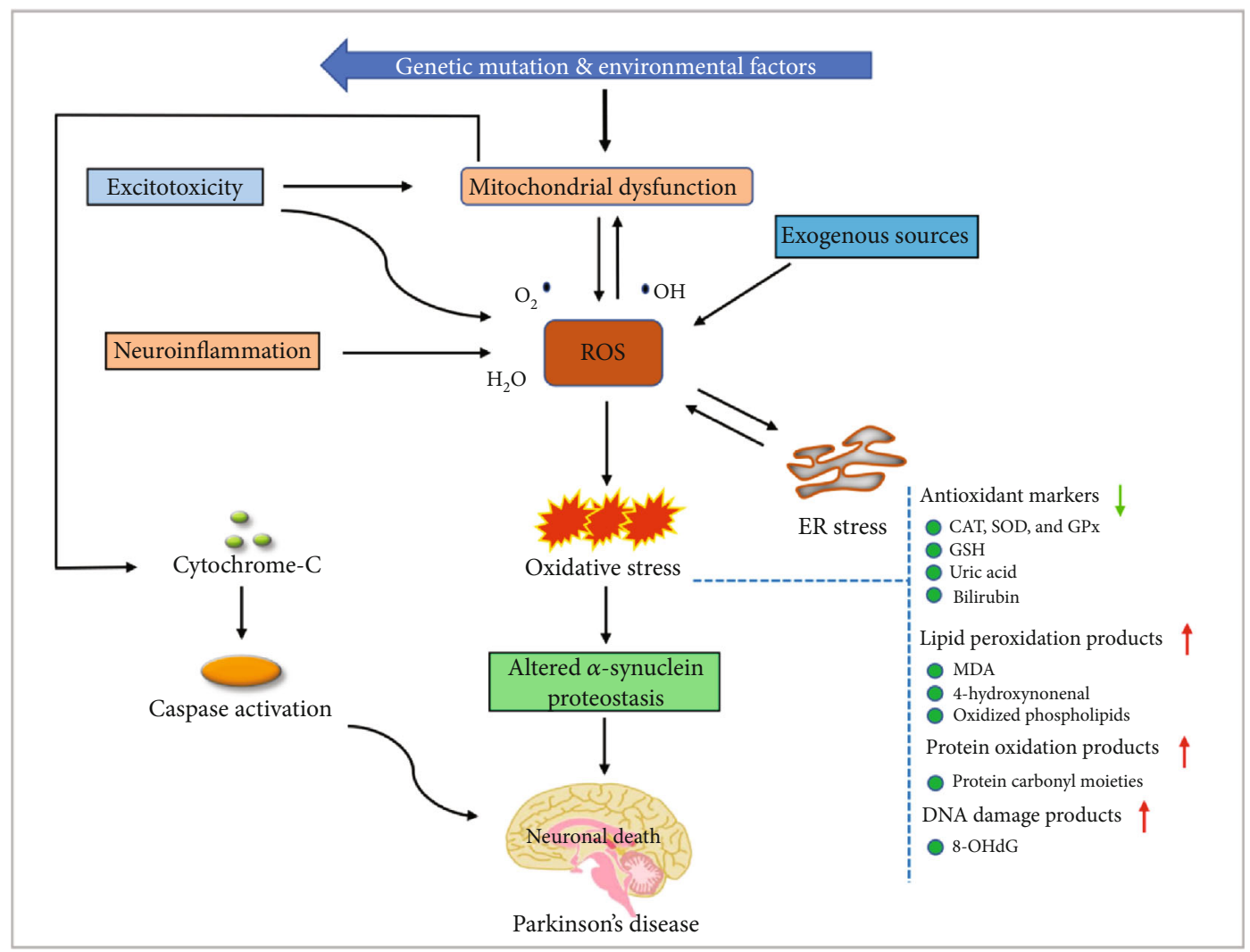

FIGURE 2: Oxidative stress and its implications in the pathogenesis of neurodegeneration in PD. When the production of ROS generation overwhelms intracellular antioxidant defenses, brain cells are exposed to oxidative stress, which may lead to mitochondrial dysfunction and further ROS production. Oxidative stress impairs the protein degradation system and hinders the clearance and results in the subsequent deposition of misfolded protein, which in turn results in lipid peroxidation, protein oxidation, and DNA damage, leading to neuronal death. These events constitute the pathological basis of PD. CAT: catalase; SOD: superoxide dismutase; GPx: glutathione peroxidase; GSH: glutathione; MDA: malondialdehyde; 8-OHdG: 8-hydroxydeoxyguanosine.

ROS, and cell death in $\mathrm{H}_{2} \mathrm{O}_{2}$ - and 6-OHDA-exposed wildtype, but not mutant cells $[91,92]$. In animal models, DJ-1 overexpression has been shown to protect against dopaminergic neuronal degeneration in mice exposed to MPTP and rats exposed to 6-OHDA in wild-type, but not mutant murine models $[91,93]$. Similarly, novel drugs activating DJ-1 protected against rotenone- and 6-OHDA-induced degeneration of SN dopaminergic neurons in murine PD models [94]. The findings of the previous studies suggested that these neuroprotective effects of DJ-1 may diminish ROS production in response to local L-calcium channels' pacemaking activity in dopaminergic neurons through regulation of mitochondrial functions [95]. Overall, the excess ROS and RNS are generated when autophagy and mitophagy are impaired. Consequently, in the present study, we explore evidence of the autophagybased neuroprotective effects of some natural phytochemicals in experimental PD.

\section{Oxidative Stress and Antioxidant Defense Mechanisms}

Nrf2 is a ubiquitous transcription factor that binds to the ARE in the maintenance of intracellular homeostasis and protects cells from harmful chemical agents by inducing the detoxifying agent and responding to oxidative stress. Under normal conditions, three known ubiquitin ligase systems, E3 ubiquitin ligase Hrd1, glycogen synthase kinase (GSK3 $\beta$ ), and Kelch-like ECH-associated protein 1 (Keap1), contribute extensively to the degradation of Nrf2 [96]. The electrophilic nature of these three activator genes enables the modification of thiol group (-SH) groups in Keap1 via oxidation, alkylation, and reduction processes [97]. Under oxidative stress condition, the Nrf2-ubiquitin ligase system's interaction is disturbed, and newly synthesized Nrf2 is transported into the nucleus, where it binds to the ARE region of specific target genes [98]. These targets are typically considered to be antioxidant genes; however, in recent decades, several scientific findings have demonstrated the extensive involvement of Nrf2 in the regulation of various physiological functions through its antioxidant, anti-inflammatory, autophagic, detoxifying, and proteasomal actions $[99,100]$. Some of the physiological functions are directly or indirectly linked with PD pathogenesis. Previous research has examined the Nrf2 signaling-mediated antioxidant protection of neurons in numerous PD models, finding that Nrf2 directly regulates the activities of catalase (CAT), sulfiredoxin-1 (SRXN1), NAD(P) H:quinone oxidoreductase-1 (NQO1), superoxide dismutase 2 (SOD2), heme oxygenase-1 (HO-1), peroxiredoxins 3 
and 5 (PRDX3 and PRDX5), and enzymes involved in GSH metabolism [101-103]. Genetic variation in the Nrf2 gene has been associated with PD progression [98]. Recently, microchip analysis revealed that Nrf2-dependent signaling cascades play a vital role during PD; this role was well illustrated in various types of tissues of patients with PD. The expression of 31 genes that contain ARE sequences in the promoter decreased significantly, while the expression of Nrf2 increased in all of the tissue samples of PD patients [104].

Recently, several in vitro and in vivo findings provided evidence for a possible protective role of the Nrf2-ARE pathway in $\mathrm{PD}$. For instance, apomorphine, which acts a dopamine $\mathrm{D}(1) / \mathrm{D}(2)$ receptor agonist, activates the Nrf2-ARE signaling pathway to protect against ROS-mediated damage, thereby bolstering the cellular defense system. Pretreatment of SH-SY5Y cells with apomorphine stimulated the translocation of Nrf2 into the nucleus and the transactivation of the ARE. The expression of HO-1 was induced by apomorphine in a dose-dependent manner. Moreover, the authors found that the activation of the ARE and the induction of HO-1 mRNA caused by apomorphine were suppressed in the presence of intracellular ROS in 6-OHDA-induced SHSY5Y cells [105]. Transplantation of astroglia cells overexpressing Nrf2 into mouse brain showed a significant decline in vulnerability to 6-OHDA-mediated neurotoxicity [106]. Similarly, astrocytic Nrf2 overexpression diminished MPTPmediated toxicity in mice, demonstrating that astrocytic modulation of the Nrf2-ARE pathway indicates decreased or attenuated neuronal death in PD [107]. Deprenyl, a drug used in the treatment of PD, was shown to stimulate Nrf2 activity as part of its cytoprotective mechanism of action [108]. Another study reported that flavonoid luteolin effectively protects against the mitochondrial toxin $\mathrm{MPP}^{+}$by activation of the Nrf2 pathway in cultured rat neuronal PC12 and glial C6 cells [109]. In a more recent study, the knockdown of Nrf2 nullified this protective effect, showing that luteolin-triggered protection is mediated solely through Nrf2. Similarly, the Nrf2 transcriptional activator tBHQ protected against neurotoxin deltamethrin-induced PC12 in a cellular PD model [110]. In a very recent study using an MPTP-induced PD model, uric acid increased Nrf2responsive genes, including g-glutamate-cysteine ligase catalytic subunit (g-GCLC), HO-1, NQO1, and mRNA, and protein expressions of Nrf2 [111]. Sulforaphane, a natural phytochemical, significantly attenuated cell damage and reversed the reduction of Nrf2, HO-1, and NQO1 expression induced by $\mathrm{MPP}^{+}$neurotoxicity; these protective effects are due in part to the activation of the Nrf2-ARE signaling pathway [112]. A similar protective effect against the decreased levels Nrf2 and Keap1 caused by 6-OHDA has been reported in rats treated with puerarin; the puerarin decreased lipid peroxidation and oxidative stress in the $\mathrm{SN}$, significantly increased brain-derived neurotrophic factor (BDNF) expression, and activated the Nrf2/ARE signaling pathway [113]. Another study reported that dietary bioflavonoid pinostrobin significantly enhanced Nrf2 expression and nuclear accumulation, improved ARE promoter activity, and upregulated expression of HO-1. Interestingly, authors then found that pinostrobin promoted phosphorylation of PI3K/AKT and ERK, and pharmacological inhibition of PI3K/AKT or ERK signaling diminished pinostrobin-induced Nrf2/ARE activation and neuroprotective actions against $\mathrm{MPTP} / \mathrm{MPP}^{+}$induced neurotoxicity in PD models [114]. Together, these findings strongly indicate that treatment of PD via activation of the Nrf2-ARE signaling pathway may be possible. Moreover, these studies demonstrate the protective effects of naturally derived antioxidant phytochemicals on Nrf2-ARE-induced antioxidant protection in experimental models of PD.

\section{Structure-Activity Relationship between Phytochemicals and Neuroprotection in PD}

Recent reports have shown that polyphenols, terpenoids, flavonoids, ascorbic acid, alpha-tocopherol, catechins, and beta-carotene may offer a potential neuroprotective effect and play a role in alleviating the progression of PD [39, 115]. Polyphenolic compounds have been found in a variety of edible and nonedible plants; to date, approximately 8000 different flavonoids have been identified in different organs of plant tissues $[116,117]$. ROS generation and RNS in mitochondria and nicotinamide adenine dinucleotide phosphate (NADPH) oxidase can trigger oxidative damage harmful to cells and decrease antioxidant defense, resulting in ageassociated NDDs such as PD [118, 119]. Moreover, free radical-mediated oxidation of nucleic acids, lipid oxidation, and oxidation of proteins can damage cell membranes and trigger protein crosslinking [120]. The health benefits of flavonoids and terpenoids are attributed to their antioxidant and chelating abilities. Through their capacity for oxidation, terpenoids and flavonoids have confirmed unique neuroprotective effects $[39,121]$. Flavonoids give a proton to form a phenoxyl radical and a singlet oxygen and scavenge superoxide as well as hydroxyl and peroxyl radicals by the release of an additional proton. Indeed, a complex structure was formed by the diol group with copper, various transition metal ions, and ferric iron, which plays a crucial role in preventing the production of ROS [122]. Furthermore, recent research revealed that flavonoids, terpenoids, and nonflavonoids chelate copper and iron ions and reduce the generation of free radicals [123].

The structure required for neuroprotective activity is not always linked with antioxidant properties [124]. Previous studies have demonstrated that EGCG, TF, and EA and its derivatives have good free-radical-scavenging effects and relatively high hydrophobic properties; thus, these compounds showed much higher antioxidant activity $[125,126]$. These results suggest the relationship of independent antioxidant mechanisms in their neuroprotective activity. Furthermore, glutathione reductase (GR), GPx, CAT, SOD, and glutathione S-transferases (GSTs) are polyphenols that upregulate ROS-scavenging enzymes [127]. Polyphenols may enhance the levels of these antioxidants by activation of cellular signaling pathways [128]. Phenolic acids of FA, EA, and CA have 4-hydroxy and electron-donating 3-methoxy groups on the benzene ring and can scavenge hydroxyl, peroxynitrite, and superoxide and terminate radical chain reactions. 
These phenolic acids effectively bind to the lipid bilayer and prevent lipid peroxidation with an adjacent unsaturated carbon-carbon double bond $[129,130]$. There is evidence that phenolic acids and their ethyl ester derivatives can upregulate protective genes, such as heat shock protein-70 (HSP-70), HO-1, and ERK1/2, which are beneficial therapeutic and preventive agents in NDDs, particularly in PD $[129,131]$.

The chemical properties of chrysin, due to a lack of B- and C-ring oxygenation, are linked with several pharmacological properties that range from antioxidant to antiinflammatory effects [132]. Differences in the chemical structure of flavones have been shown to enhance the activity of antioxidant enzymes and to provide an inhibitory effect on the proinflammatory mediator of COX-2 expression [133]. In addition, xanthine oxidase inhibitory assay has shown that chrysin significantly suppresses xanthine oxidase production of ROS [134, 135], and amentoflavone and blueberry potentially inhibit iNOS expression [136]. Interestingly, in rodent models, $10-100 \mu \mathrm{M}$ concentrations are required to display the potential antioxidant and anti-inflammatory activities of polyphenols [134, 135].

\section{Neuroprotective Roles of Phytochemicals: Antioxidant, Anti-Inflammatory, and Other Signaling Pathways}

6.1. Neuroprotective Mechanisms of Chrysin in PD. Chrysin is a natural polyphenolic compound known as a flavonoid; flavonoids are ubiquitous in vegetables, fruits, mushrooms, blue passion flowers, plants, and especially in honey [137]. Numerous epidemiological, cellular, and animal studies have substantiated the potential health benefits of flavonoids. The main sources of flavonoids in Westernized countries-specifically Australia, the United States, and many European nations-are tea, fruit or vegetable juices, blue passion flowers, and wine, with the estimated total intake of flavonoids ranging from $200 \mathrm{mg} /$ day in Australia [138] to $500 \mathrm{mg} /$ day in parts of Europe where tea consumption is high [139]. In countries such as Spain, Poland, Mexico, and Greece, where diets are rich in citrus fruits and wine, dietary flavanones can range from $30 \mathrm{mg} /$ day (Greece) to $170 \mathrm{mg} /$ day (Poland) [139-142]. Similarly, in Asian countries (China, Japan, and Korea), dietary flavanone intake ranged from $36 \mathrm{mg} /$ day (Korea) to $5 \mathrm{mg} /$ day (China) [143-146]. Chrysin has been explored for its neuroprotective effects, which are attributable to its antioxidant, anti-inflammatory, and other pharmacological properties. The various therapeutic activities of chrysin depend on its bioavailability and attainable concentrations in cells and target tissues in rodents. Following administration of $400 \mathrm{mg}$ of chrysin to human subjects, bioavailability was found to be extremely low due to rapid metabolism, poor intestinal absorption, and rapid excretion [147]. Scientific evidence has established that chrysin is found conjugated approximately $99 \%$ in the plasma protein binding [147]. However, it was reported that chrysin is considered to have very low oral bioavailability and a distribution volume of approximately $0.003 \%-0.02 \%$ [147]. Furthermore, a recent investigation demonstrated that chrysin can cross biological membranes easily; however, extensive sulfation and glucuronidation in the intestinal cells limit its absorption [148]. Although chrysin is considered safe for human consumption at a daily dose of $500 \mathrm{mg}$ to $3 \mathrm{~g}$ without any toxic effects, higher doses may induce undesirable side effects [149]. Chrysin is especially important for polyphenols targeting the brain; penetration of the blood-brain barrier (BBB) is crucial for their therapeutic benefits [150].

The neuroprotective activity of chrysin shows that increases in DA levels in both in vitro and in vivo experiments are inversely associated with dopaminergic neuronal loss (Table 1) [151, 152]. It was found that chrysin enhanced DA levels by acting as an Monoamine oxidase B (MAO-B) inhibitor in $\mathrm{MPP}^{+}$- and MPTP-treated CGN cells and mouse models of PD [152]. In this way, chrysin treatment can increase DA levels and subsequently suppress DA metabolism in the ST through the inhibition of MAO-B [152]. Several neurotoxin-induced in vivo experiments also demonstrated that chrysin increased DA, 3,4-dihydroxyphenylacetic acid (DOPAC), and homovanillic acid (HVA) levels $[153,154]$. Moreover, chrysin administration very significantly attenuated the cognitive dysfunction and motor impairment in these animals as evaluated by passive avoidance, rotational behavior, and Barnes maze tests [154], as well as beam walk and horizontal and vertical grid tests [153]. Therefore, chrysin presents as a potential diseasemodifying agent that may also prove beneficial for symptom relief in PD.

Several preclinical studies have revealed that chrysin provides antioxidant effects by reducing oxidative stress and modulating or boosting cellular antioxidant enzyme levels, thus decreasing the lipid peroxidation triggered by numerous oxidative insults [155-157]. In aged mice, chrysin administration significantly blocked the elevation of ROS levels and boosted antioxidant enzyme activities [158]. In the MPTPinduced PD mouse model, chrysin pretreatment diminished oxidative stress as evaluated by lipid peroxidation levels; the pretreatment also inhibited reduction of SOD activity and GSH content in the SN region [153]. In agreement with these results, chrysin administration inhibited the increase of $\mathrm{NO}$ and NADPH oxidase activity and prevented the reduction of GSH in the ST of 6-OHDA-treated mice [154]. Additionally, chrysin treatment attenuated 6-OHDA-induced decreases in the levels of $\mathrm{Na}^{+}$and $\mathrm{K}^{+}$-ATPase in the ST of mice. $\mathrm{Na}^{+}$and $\mathrm{K}^{+}$-ATPase play a central role in maintaining ionic gradients and neuronal excitability and are more susceptible to oxidative damage [159].

RNS generated by iNOS may be significantly associated with oxidative stress in $\mathrm{PD}$, and excessive production of $\mathrm{NO}$ can lead to loss of dopaminergic neurons and impaired motor functions [160]. NADPH oxidase activation can stimulate ROS and plays a vital role in dopaminergic neurodegeneration [161]. In this regard, pretreatment of PC12 cells with chrysin may reduce the intracellular NO level, decrease iNOS expression, and subsequently inhibit NF- $\kappa \mathrm{B}$ phosphorylation and decrease its transcriptional activity induced by 6-OHDA neurotoxicity [162]. In particular, chrysin administration significantly enhanced the anti-inflammatory markers interleukin-4 (IL-4) and interleukin-10 (IL-10), diminished 
TABLE 1: Promising studies of antioxidant phytochemicals for the management of PD.

\begin{tabular}{|c|c|c|c|c|c|}
\hline Phytochemicals & Studied materials & Dose & Neurotoxins & Physiological effects & References \\
\hline \multirow{3}{*}{ Chrysin } & $\begin{array}{l}\text { Male C57BL/6J } \\
\text { mice }\end{array}$ & $10 \mathrm{mg} / \mathrm{kg}$ & 6-OHDA & $\begin{array}{c}\uparrow \text { Behavioral functions } \\
\uparrow T H-\text { positive cells in the SN and ST } \\
\uparrow \mathrm{DA}, \text { DOPAC, and HVA levels }\end{array}$ & {$[151]$} \\
\hline & $\begin{array}{c}\text { Male C57BL/6 } \\
\text { mice }\end{array}$ & $\begin{array}{c}50 \text { and } \\
100 \mathrm{mg} / \mathrm{kg}\end{array}$ & MPTP & $\begin{array}{c}\uparrow \mathrm{DA} \text { and its metabolites } \\
\uparrow \mathrm{AKT} / \mathrm{GSK} 3 \beta / \mathrm{MEF} 2 \mathrm{D} \text { pathway } \\
\downarrow \text { MAO-B activity }\end{array}$ & {$[152]$} \\
\hline & $\begin{array}{l}\text { Male C57BL/6J } \\
\text { mice }\end{array}$ & $\begin{array}{l}50,100, \text { and } \\
200 \mathrm{mg} / \mathrm{kg}\end{array}$ & MPTP & $\begin{array}{c}\uparrow \mathrm{BDNF} \text { and GDNF protein expression } \\
\downarrow \mathrm{IL}-10, \mathrm{IL}-6, \mathrm{TNF}-\alpha \text {, and NF- } \kappa \text { B protein } \\
\text { expression }\end{array}$ & [153] \\
\hline \multirow{2}{*}{ Vanillin } & $\begin{array}{c}\text { Male Wistar } \\
\text { albino rats }\end{array}$ & $\begin{array}{l}5,10, \text { and } \\
20 \mathrm{mg} / \mathrm{kg}\end{array}$ & LPS & $\begin{array}{c}\downarrow \text { iNOS, COX-2, IL- } 1 \beta \text {, and IL-6 protein } \\
\text { expression } \\
\downarrow \text { ERK } 1 / 2 \text {, p38, and NF- } \kappa \text { B signaling } \\
\downarrow \text { Microglia activation }\end{array}$ & {$[167]$} \\
\hline & $\begin{array}{c}\text { Male Wistar } \\
\text { albino rats }\end{array}$ & $\begin{array}{l}5,10, \text { and } \\
20 \mathrm{mg} / \mathrm{kg}\end{array}$ & Rotenone & $\begin{array}{c}\uparrow \text { Striatal DA and its metabolite levels } \\
\uparrow \text { Behavioral function } \\
\downarrow \text { Cyto-C, Bax, and caspase protein expression } \\
\uparrow B c l-2 \text { protein expressions }\end{array}$ & {$[168]$} \\
\hline \multirow[b]{2}{*}{ Asiatic acid } & $\begin{array}{c}\text { Male C57BL/6 } \\
\text { mice }\end{array}$ & $\begin{array}{l}20,40 \text {, and } \\
80 \mathrm{mg} / \mathrm{kg}\end{array}$ & MPTP & $\begin{array}{c}\uparrow \text { Striatal DA levels } \\
\uparrow \text { Striatal TH, TLR4, BDNF, and GFAP protein } \\
\text { expression } \\
\downarrow \alpha \text {-Synuclein and lowered AIF protein } \\
\text { expression }\end{array}$ & [169] \\
\hline & $\begin{array}{c}\text { Male Wistar } \\
\text { albino rats }\end{array}$ & $\begin{array}{l}100 \mathrm{mg} / \mathrm{kg} \\
\text { (in vivo) } \\
\text { and } 0.1- \\
10 \mathrm{nM} \\
\text { (in vitro) }\end{array}$ & $\begin{array}{c}\mathrm{MPTP} / \mathrm{p} \\
\text { and } \mathrm{MPP}^{+}\end{array}$ & $\begin{array}{c}\uparrow \text { Motor functions } \\
\uparrow \mathrm{PI} 3 \mathrm{~K}, \text { Akt, GSK-3 } \beta \text {, and mTOR } \\
\text { phosphorylation } \\
\uparrow \text { TrKB protein expression } \\
\downarrow \text { NLRP3 inflammasome expression in } \\
\text { microglia cells }\end{array}$ & {$[170]$} \\
\hline \multirow{3}{*}{ Ferulic acid } & $\begin{array}{l}\text { Male Wistar } \\
\text { albino rats }\end{array}$ & $100 \mathrm{mg} / \mathrm{kg}$ & 6-OHDA & $\begin{array}{c}\downarrow \text { Mitochondrial Drp1 expression } \\
\uparrow \mathrm{PGC1} \alpha \text { gene and protein expression } \\
\uparrow \mathrm{Mfn} 2 \text { and mitochondrial dynamics }\end{array}$ & {$[171]$} \\
\hline & $\begin{array}{c}\text { Male C57BL/6 } \\
\text { mice }\end{array}$ & $100 \mathrm{mg} / \mathrm{kg}$ & Rotenone & $\begin{array}{c}\uparrow \text { Motor function } \\
\uparrow H S P-70 \text { protein expression } \\
\uparrow T H-\text { positive fibers in corpus striatum }\end{array}$ & {$[172]$} \\
\hline & $\begin{array}{c}\text { Male C57BL/6 } \\
\text { mice }\end{array}$ & $\begin{array}{l}20 \mathrm{mg} / \mathrm{kg} \text { and } \\
\text { muscle } \\
\text { exercise }\end{array}$ & MPTP & $\begin{array}{c}\uparrow \text { Motor behavior } \\
\uparrow \text { CAT, SOD, GPx, and GSH activity } \\
\downarrow \text { TBARS activity } \\
\uparrow \text { Activation of the Nrf2 signaling }\end{array}$ & [173] \\
\hline \multirow{3}{*}{ Thymoquinone } & $\begin{array}{c}\text { Male C57BL/6 } \\
\text { mice }\end{array}$ & $40 \mathrm{mg} / \mathrm{kg}$ & MPTP & $\begin{array}{c}\uparrow \mathrm{CAT}, \mathrm{SOD}, \mathrm{GPx} \text {, and GSH activity } \\
\downarrow \text { TBARS activity } \\
\downarrow \text { iNOS, COX-2, IL-1 } \beta \text {, and IL-6 protein } \\
\text { expression }\end{array}$ & {$[174]$} \\
\hline & $\begin{array}{c}\text { Male Wistar } \\
\text { albino rats }\end{array}$ & $\begin{array}{c}7.5 \text { and } \\
15 \mathrm{mg} / \mathrm{kg}\end{array}$ & Rotenone & $\begin{array}{c}\uparrow \text { Behavioral functions } \\
\uparrow \text { Parkin, Drp1, TH-positive cells in the SN } \\
\text { and ST } \\
\uparrow \text { DA, DOPAC, and HVA levels }\end{array}$ & [175] \\
\hline & $\begin{array}{l}\text { Male Wistar } \\
\text { albino rats }\end{array}$ & $\begin{array}{c}5 \text { and } \\
10 \mathrm{mg} / \mathrm{kg}\end{array}$ & 6-OHDA & $\begin{array}{c}\uparrow \text { Behavioral functions } \\
\uparrow \text { DA level in the SN } \\
\downarrow \text { MDA level }\end{array}$ & {$[176]$} \\
\hline \multirow[b]{2}{*}{ Ellagic acid } & $\begin{array}{l}\text { Male Wistar } \\
\text { albino rats }\end{array}$ & $50 \mathrm{mg} / \mathrm{kg}$ & 6-OHDA & $\begin{array}{l}\uparrow \text { Motor function and electrophysiological } \\
\text { performance } \\
\uparrow C A T, \text { SOD, GPx, and GSH cerebral activity }\end{array}$ & [177] \\
\hline & $\begin{array}{c}\text { Male Wistar } \\
\text { albino rats }\end{array}$ & $50 \mathrm{mg} / \mathrm{kg}$ & 6-OHDA & $\begin{array}{c}\uparrow \mathrm{CAT}, \mathrm{SOD}, \mathrm{GPx}, \text { and GSH cerebral activity } \\
\downarrow \text { TBARS activity } \\
\downarrow \text { MAO-B activity } \\
\uparrow \mathrm{ER} \beta / \mathrm{Nrf} 2 / \mathrm{HO}-1 \text { signaling cascade }\end{array}$ & [178] \\
\hline
\end{tabular}


Table 1: Continued.

\begin{tabular}{|c|c|c|c|c|c|}
\hline Phytochemicals & Studied materials & Dose & Neurotoxins & Physiological effects & References \\
\hline \multirow[b]{3}{*}{ Caffeic acid } & $\begin{array}{l}\text { Drosophila } \\
\text { melanogaster }\end{array}$ & $\begin{array}{l}0.5,1 \text {, and } \\
2 \mathrm{mg} / \mathrm{g}\end{array}$ & Paraquat & $\begin{array}{c}\uparrow \mathrm{CAT}, \mathrm{SOD}, \mathrm{GPx} \text {, and GSH cerebral activity } \\
\downarrow \text { TBARS activity } \\
\uparrow \mathrm{Nrf2-Keap} 1 \text { signaling }\end{array}$ & {$[179]$} \\
\hline & $\begin{array}{l}\text { A53T transgenic } \\
\text { mice }\end{array}$ & $5 \mathrm{mg} / \mathrm{kg}$ & $\begin{array}{l}\mathrm{A} 53 \mathrm{~T} \mathrm{Tg} \\
\text { mice }\end{array}$ & $\begin{array}{c}\downarrow \mathrm{A} 53 \mathrm{~T} \alpha \text {-synuclein } \\
\uparrow \mathrm{Bcl}-2 \text {-mediated autophagy pathway } \\
\uparrow \text { Behavioral functions }\end{array}$ & {$[180]$} \\
\hline & $\begin{array}{l}\text { Male C57BL/6 } \\
\text { mice }\end{array}$ & $\begin{array}{l}0.5,1 \text {, and } \\
2 \mathrm{~g} / \mathrm{kg}\end{array}$ & MPTP & $\begin{array}{c}\uparrow D A \text { synthesis } \\
\uparrow \text { TH-positive cells } \\
\uparrow \text { BDNF and GDNF protein expression, } \\
\text { maintained loss } \\
\downarrow \text { IL- } 1 \beta, \text { IL- } 6 \text {, TNF- } \alpha \text {, iNOS, and COX-2 } \\
\text { expression } \\
\downarrow \text { GFAP protein expression }\end{array}$ & {$[181]$} \\
\hline \multirow{4}{*}{ Epigallocatechin-3-gallate } & $\begin{array}{l}\text { Male C57BL/6 } \\
\text { mice }\end{array}$ & $50 \mathrm{mg} / \mathrm{kg}$ & MPTP & $\begin{array}{c}\uparrow \text { Iron-export protein ferroportin in SN } \\
\uparrow C A T, \text { SOD, GPx, and GSH cerebral activity } \\
\downarrow \text { TBARS activity } \\
\uparrow D A \text { synthesis }\end{array}$ & {$[182]$} \\
\hline & $\begin{array}{l}\text { Male } \mathrm{C} 57 \mathrm{BL} / 6 \mathrm{~J} \\
\text { mice }\end{array}$ & $25 \mathrm{mg} / \mathrm{kg}$ & MPTP & $\begin{array}{c}\uparrow \text { Movement behavior } \\
\uparrow T H-\text { positive cells in the SN region } \\
\uparrow \mathrm{CD} 3^{+} \mathrm{CD} 4^{+} \text {to } \mathrm{CD} 3^{+} \mathrm{CD} 8^{+} \mathrm{T} \text {-cell } \\
\text { lymphocyte ratio in the peripheral blood } \\
\downarrow \mathrm{TNF}-\alpha \text { and IL- } 6 \text { cytokine expression in serum }\end{array}$ & {$[183]$} \\
\hline & $\begin{array}{l}\text { Postmortem PD } \\
\text { tissue }\end{array}$ & $100 \mathrm{nM}$ & - & $\downarrow \alpha$-Synuclein aggregates & {$[184]$} \\
\hline & $\begin{array}{l}\text { Male C57BL/6 } \\
\text { mice }\end{array}$ & $10 \mathrm{mg} / \mathrm{kg}$ & MPTP & $\begin{array}{l}\uparrow \text { Movement behavior } \\
\downarrow \text { Microglial activation }\end{array}$ & {$[185]$} \\
\hline \multirow[t]{2}{*}{$\alpha$ - and $\beta$-Asarone } & $\begin{array}{l}\text { Male C57BL/6 } \\
\text { mice }\end{array}$ & $10 \mathrm{mg} / \mathrm{kg}$ & 6-OHDA & $\begin{array}{l}\uparrow H V A, \text { DOPAC, } 5 \text {-HIAA levels } \\
\uparrow T H-\text { positive cells in the ST region } \\
\downarrow J N K \text { and p-JNK expression } \\
\uparrow B c l-2 \text { protein expression }\end{array}$ & [186] \\
\hline & $\begin{array}{l}\text { Sprague Dawley } \\
\text { rats }\end{array}$ & $15 \mathrm{mg} / \mathrm{kg}$ & 6-OHDA & $\begin{array}{c}\uparrow \mathrm{CAT}, \mathrm{SOD}, \mathrm{GPx} \text {, and GSH cerebral activity } \\
\downarrow \text { TBARS activity } \\
\uparrow \mathrm{PERK} / \mathrm{CHOP} / \mathrm{Bcl}-2 / \text { Beclin-1 pathway } \\
\downarrow \text { GRP78 levels }\end{array}$ & {$[187]$} \\
\hline \multirow{3}{*}{ Theaflavin } & $\begin{array}{l}\text { Male C57BL/6 } \\
\text { mice }\end{array}$ & $10 \mathrm{mg} / \mathrm{kg}$ & $\mathrm{MPTP} / \mathrm{p}$ & $\begin{array}{c}\uparrow \mathrm{DAT} \text { and VMAT-2 expression } \\
\uparrow \text { Behavioral functions } \\
\uparrow \mathrm{CAT}, \mathrm{SOD}, \mathrm{GPx} \text {, and GSH cerebral activity } \\
\downarrow \text { TBARS activity }\end{array}$ & {$[188]$} \\
\hline & $\begin{array}{l}\text { Male C57BL/6 } \\
\text { mice }\end{array}$ & $10 \mathrm{mg} / \mathrm{kg}$ & $\mathrm{MPTP} / \mathrm{p}$ & $\begin{array}{c}\uparrow \text { Behavioral characterization } \\
\uparrow \mathrm{TH}-\text { positive cells in the ST region } \\
\downarrow \text { Caspase-3, caspase- } 8 \text {, and caspase-9 activity } \\
\downarrow \text { Bax expression } \\
\uparrow \text { Bcl-2 protein expressions }\end{array}$ & [189] \\
\hline & $\begin{array}{l}\text { Male C57BL/6 } \\
\text { mice }\end{array}$ & $10 \mathrm{mg} / \mathrm{kg}$ & $\mathrm{MPTP} / \mathrm{p}$ & $\begin{array}{c}\uparrow \text { Behavioral characterization } \\
\downarrow \text { IL-4 and IL-10 protein expressions }\end{array}$ & [190] \\
\hline
\end{tabular}

expression of tumor necrosis factor-alpha (TNF- $\alpha$ ), interleukin-1 $\beta$ (IL-1 $\beta$ ), and interleukin-6 (IL-6), and inhibited myeloperoxidase (MPO), COX-2, and iNOS expression, which are highly implicated in anti-inflammatory responses [163]. In a cerebral ischemic mouse model, chrysin inhibited neuroinflammation by reducing the level of IL- $1 \beta$ and TNF- $\alpha$ expression, prevented cognitive deficits, and downregulated the activation of NF- $\kappa \mathrm{B}$ [164]. In addition to inhibiting NF$\kappa \mathrm{B}$ activation and downregulating gene-related inflammation, chrysin also interacts with iNOS enzymes at a molecular level and COX-2 and decreases levels of arachidonic acidinduced prostaglandins, thromboxanes, and leukotrienes in the SN and ST of 6-OHDA- and MPTP-treated PD mice $[151,153]$. Hence, one may conclude that chrysin's protective effects may carry promising anti-inflammatory properties in PD that are at least partially mediated by $\mathrm{NF}-\kappa \mathrm{B}$ inhibition.

In an experimental model using MPTP, chrysin administered to mice was demonstrated to attenuate dopaminergic neuronal loss in the SN region [152]. Consequently, 
immunohistochemical analysis revealed that oral administration of chrysin significantly restored the loss of TH-positive cells in the ST and protected nigrostriatal morphology against 6-OHDA-induced mouse models of PD [151]. A very recent study showed that oral administration of chrysin improved locomotor activity and protected against dopaminergic neurodegeneration in MPTP-injected PD mouse models [153]. Chrysin played a neuroprotective role at a molecular level, at least in part through the suppression of proapoptotic proteins such as caspase-3, caspase-9, and $\mathrm{Bax}$, and enhanced antiapoptotic protein $\mathrm{Bcl}-2$ expression against $\mathrm{MPP}^{+}$neurotoxicity [152]. Moreover, chrysin treatment provided neuroprotectivity by regulating or restoring BDNF and glial cell-derived neurotrophic factor (GDNF) levels in the ST region in 6-OHDA- and MPTP-induced PD mouse models [151, 153]. Importantly, PD animal models showed that neurotrophic factors such as BDNF and GDNF could partially inhibit neurodegeneration as reported by previous studies $[165,166]$. Hence, the multiple protective roles of chrysin in $\mathrm{PD}$, both neuroprotective and symptom-relieving, are of great value and may open new horizons for novel therapeutic management of PD; further clinical studies are needed.

6.2. Neuroprotective Mechanisms of Vanillin in PD. Vanillin is a phenolic aldehyde compound used as an important flavoring agent worldwide. It is found in abundance in plant species and is often used in the food production, beverage, pharmaceutical, perfume, and cosmetic industries [191]. Currently, approximately $50 \%$ of worldwide synthetic vanillin production is used as an intermediate in both food and nonfood applications and in pharmaceutical industries for the production of herbicides, antifoaming agents, or drugs such as papaverine, L-dopa, L-methyldopa, and antimicrobial agents [191]. Acceptable daily intake of vanillin is consumed in the form of food and beverage worldwide, and its ubiquity suggests that nearly every human consumes vanillin-containing products. A daily intake of vanillin $10 \mathrm{mg} / \mathrm{kg}$ has been approved by the Food and Agriculture Organization of the United Nations (FAO)/WHO and European Union. For a person weighing $70 \mathrm{~kg}$, the daily recommended intake is $700 \mathrm{mg}$ of vanillin, which amounts to a minimum of $700 \mathrm{~g}$ of chocolate or $700 \mathrm{~g}$ of ice cream [192].

Vanillin has been studied extensively for its pharmacological properties, which are attributable to its structure and main bioactive metabolites, including vanillyl alcohol and vanillic acid; vanillin's bioactive properties include antioxidant, anti-inflammatory, and neuroprotective abilities [193]. Vanillin can easily penetrate the BBB and demonstrates significant brain-neuroprotective potential by enhancing the activities of antioxidant enzymes and reducing the levels of lipid peroxidation and NO production $[194,195]$. This polyphenolic flavoring agent can scavenge the $\mathrm{O}^{-}-2$ and $\mathrm{OH}$ intermediates implicated in biological membrane damage [196]. It alleviates renal oxidative stress by decreasing lipid peroxidation levels, increasing levels of enzymatic (SOD, CAT, GPx, and GSH) and nonenzymatic antioxidants (vitamin $\mathrm{C}$ and nonprotein thiol), and protecting against DNA damage and histopathological changes in maneb-induced mice [197]. Another study demonstrated the neuroprotective properties of vanillin in a rotenone-induced PD model. Administration of vanillin in SH-SY5Y cells inhibited rotenone-induced ROS generation, mitochondrial dysfunction, and caspase activation and reduced the expression of signaling molecules [198].

A previous study elucidated the relationship between striatal DA depletion and motor impairments [199]. Furthermore, intraperitoneal injection of rotenone displayed a significant diminishment in locomotion activity, akinetic movement, and cataleptic ability [200]. Oxidative stressmediated degeneration of neurons was further exacerbated by high levels of DA metabolism, greater prevalence of glial cells, and low levels of antioxidant enzyme activity in the SN and ST regions [201]. The successful antioxidant actions of vanillin are due to its structure by reducing the levels of lipid peroxidation and enhancing the antioxidant enzyme activities $[168,202]$. A recent study determined that vanillin treatment increases the striatal DA and its metabolites, subsequently improving behavioral function. It has also been reported to prevent Cyto- $\mathrm{C}$ release, diminish Bax expression, inhibit caspase activation, and enhance $\mathrm{Bcl}-2$ expression in rotenone-induced rat models of PD [168]. Vanillin has also exhibited improvement in chronic stress-induced rat models by reducing the depressive-like motor symptoms via elevation of serotonin and DA levels in brain tissue [203].

Other study findings recently revealed that mice that inhaled vanillin had a decreased pain response to the hot plate test; no significant differences were observed between the inhaled vanillin group and the control group in the tail suspension, Y-maze, forced swimming, open field, and aggression tests. These results suggested that inhaled vanillin has potential antinociceptive properties similar to other routes of administration [204]. In LPS-lesioned PD models, oral administration of vanillin reduced IL- $1 \beta$, IL- 6 , and TNF- $\alpha$ expression through inhibition of the p38-MAPK signaling pathway, suppressing activation of NF- $\kappa \mathrm{B}$ and inflammatory genes like iNOS that produce NO and COX-2. Consequently, LPS-lesioned rats experienced increased degeneration of dopaminergic neurons and decreased motor function, and microglial activation triggered in the $\mathrm{SN}$ and ST was restored or improved significantly following vanillin treatment [167]. Similar findings also confirmed that vanillin reduced the inflammatory mediators of iNOS and COX-2, as well as the mRNA expression levels of proinflammatory cytokines. In addition, vanillin effectively inhibited the phosphorylation of MAPK signaling and NF- $\kappa$ B activation in LPSlesioned microglia cells [205]. Together, these data suggest the neuroprotective and anti-inflammatory role of vanillin in protecting dopaminergic neurons and improving behavioral function by inhibiting oxidative stress, inflammation, and apoptosis; thus, it is possible that vanillin may act as a natural therapeutic drug for PD.

6.3. Neuroprotective Mechanisms of Asiatic Acid in PD. Asiatic acid (AA), a naturally pentacyclic triterpenoid, shows promise as neuroprotective drug candidate due to its several pharmacological properties. Interestingly, a number of bioactive components of AA were found to have therapeutic 
potential in curing various diseases [206, 207]. AA exhibited the ability to modulate several enzymes, growth factors, receptors, transcription factors, apoptotic proteins, and cell signaling molecules $[208,209]$. In experimental studies, AA has displayed a wide range of biological activities, including antioxidant, hepatoprotective, antidiabetic, anticancer, antiinflammatory, and neuroprotective properties [210-213]. Recently, AA tromethamine-loaded solid lipid nanoparticles have been shown to prevent proteolytic degradation and to facilitate sustained release of the drug, thereby improving its bioavailability [214]. Another study injected formulations containing glutathione-conjugated BSA nanoparticles of AA intravenously into rats at a dose of $75 \mathrm{mg} / \mathrm{kg}$; after 5 hours, the nanoformulation displayed ten times greater bioavailability in the brain than AA alone [215].

AA provides neuroprotection by maintaining the stability of the $\mathrm{BBB}$ and by protecting mitochondrial functions. A recent in vitro study showed that AA administration reduced intracellular mitochondrial ROS production and altered MMPs to regulate mitochondrial function, subsequently decreasing NLRP3 inflammasome expression in microglia cells [216]. In addition, AA treatment directly improved SH-SY5Y cell viability and maintained mitochondrial function in an $\mathrm{MPP}^{+}$-induced PD model [216]. Furthermore, administration of AA attenuated ROS overproduction, mitochondrial dysfunction, and apoptotic expressions of proapoptotic and antiapoptotic indices in a rotenoneinduced SH-SY5Y PD model [217]. A similar study reported that AA pretreatment protected cell viability, inhibited the upregulation of voltage-dependent anion channel mRNA and protein expression levels, and prevented MMP damage following exposure to $\mathrm{H}_{2} \mathrm{O}_{2}$ [218]. Further recent in vitro and in vivo experiments also observed that the administration of AA significantly decreased apoptotic cell death, lessened mitochondrial ROS production, stabilized MMPs, and promoted the expression of PGC1 $\alpha$ and Sirt1 to mitigate toxicity induced by glutamate in a dose-dependent manner. In the in vivo model, oral administration of AA significantly attenuated cognitive deficits, decreased lipid peroxidation level, and restored GSH content and SOD activity in the hippocampus and cortex; subsequently, AA effectively attenuated glutamate-induced neuronal damage of the pyramidal layer in the CA1 and CA3 regions [219].

Moreover, the anti-inflammatory activity of AA significantly decreases the level of MDA, NO, and inflammatory mediators iNOS and COX-2 and has also been shown to inhibit NF- $\kappa \mathrm{B}$ activation in paw edema by increasing the antioxidant enzyme levels of CAT, GPx, and SOD in $\lambda$-carrageenan-induced mice [220]. In the MPTP/p-induced PD mouse model, AA administered was shown to improve behavioral function, enhance DA levels, and increase expressions of neurotrophic factors and tyrosine kinase receptors (TrKB). Moreover, AA significantly inhibited phosphorylation of P38, JNK, and ERK protein expression and significantly increased phosphorylated PI3K, Akt, GSK-3 $\beta$, and mTOR activation [170]. Indeed, experimental studies showed that AA treatments increased striatal DA levels and upregulated striatal TH, TLR4, BDNF, and GFAP expression, subsequently decreasing striatal upregulation of $\alpha$-synuclein, apoptotic markers, and Bcl-2 expression in MPTP-induced PD-like neurotoxicity in mice. In addition, posttreatment of AA significantly suppressed NF- $\kappa$ B activation [169]. Interestingly, recent studies have also found that AA prevented decreases in Nrf2 expression, counteracted the downregulation of neurogenesis within the hippocampus, and mitigated memory deficits produced by 5 -fluorouracil through inhibiting oxidative stress and boosting the antioxidant defense system [221]. These novel findings suggest that AA may be developed as an agent for PD prevention or therapy.

6.4. Neuroprotective Mechanisms of Ferulic Acid in PD. Ferulic acid (FA) is a natural phenolic phytochemical commonly found in apples, oranges, peanuts, wheat, rice, barley, coffee, and many other dietary sources [222]. Investigations have confirmed the beneficial effect of FA-rich foods and drinks. Recent reports revealed that consumption of vegetables, fruits, and cereals is beneficial in the prevention of diabetes, cancer, obesity, cardiovascular disease, AD, and PD [129, $223,224]$. In Japan, FA has been approved as a food additive and is used as a natural antioxidant in foods, beverages, and cosmetics. In the United States and most European countries, numerous medical essences and natural extracts of herbs, coffee, vanilla beans, spices, and other botanicals are selected for their high content of FA and are added to foods as an FDA-approved antioxidant concoction [225]. Accurate nutritional surveys about FA intake are lacking, but consumption of FA from food sources can be estimated at a daily intake of approximately $200-1000 \mathrm{mg}$ per person [222, 226, 227].

In recent years, scientific findings have reported the health benefits of FA, which exhibits low toxicity and possesses several pharmacological properties including immunomodulatory, antioxidant, anti-inflammatory, antiapoptotic, anticancer, antidiabetic, and neuroprotective functions. FA achieves these functions through inhibition of lipid peroxidation and ROS generation by its phenolic hydroxyl group. Moreover, FA has been reported to downregulate the expression of enzymes that promote inflammation $[129,222]$. The physiological benefits of FA depend on its bioavailability for absorption and consequent interaction with target tissues. Recent preclinical investigations estimated that for subjects who consumed phenolic acidcontaining drinks, vegetables, and fruits in the daily diet, total daily intake of polyphenols equaled about $1 \mathrm{~g}$ [227]. In animal models, FA has shown greater bioavailability compared to other various phenolic ingredients [222, 228].

Based on its structural similarity to another phenolic compound, salicylic acid, that can enter the brain, it is speculated that FA can easily penetrate the BBB. It has also been reported to be a successful neuroprotective agent [229]. Recent in vitro and in vivo findings have demonstrated that FA exhibited increased levels of protective HO-1 activity in SH-SY5Y cells, upregulated the levels of CAT, SOD, GPx, and GSH, and reduced the lipid peroxidation level in MPTP-injected PD mouse models, which confirms FA's potential antioxidant effects in the prevention of PD [230]. In another study, FA led to upregulation of antioxidant enzyme status through induced HO-1 gene expression, 
enhanced ARE promoter activity, promoted ERK1/2 phosphorylation, and Nrf2 translocation in PC12 cells exposed to lead acetate [231]. Moreover, oxidative stress stimulated by 6-OHDA and rotenone was alleviated effectively by FA treatment via its ability to scavenge radicals generated by increased lipid peroxidation, decreased antioxidant GSH content, and mitochondrial oxygen radical production, thus substantiating FA's preventive effect against oxidative damage in PD [171, 232].

Anti-inflammation has been proposed as one of the main mechanisms underlying FA-induced neuroprotection in PD, and several studies have shown that FA can potentially inhibit neuroinflammation and neuronal apoptosis in NDDs. Furthermore, histological findings revealed that FA administration suppressed microglial cell activation and the $\mathrm{Bax} / \mathrm{Bcl}-$ 2 ratio, reflecting a reduction in inflammation and apoptosis, respectively. It was also discovered that FA effectively prevented MPTP-induced neuronal loss-triggered declines in behavioral function and motor coordination [233]. Additionally, FA reduced proinflammatory cytokines and inflammatory mediators such as iNOS and COX-2. Further, the results confirmed that FA mitigates activation of microglial and astrocytes by a remarkable reduction in GFAP and Iba1 hyperactivity [232]. In addition to their role in mitochondrial propagation and function, dynamin-related protein 1 (Drp1) and mitofusin 2 (Mfn2) have also been linked with excitotoxicity and are thought to play a vital role in programmed cell death [234]. In fact, enhanced expression of Drp1 has been linked to neuronal damage in animal models of PD [235]. Moreover, the study's author reported that FA administration attenuated 6-OHDA-induced morphological changes and DNA damage and blocked caspase activity. FA also reduced mitochondrial expression of Drp1 and increased expression of the PGC1 $\alpha$ gene and protein, thereby regulating expression of its downstream target Mfn2 and restoring mitochondrial dynamics in 6-OHDA-lesioned PD animal models [171]. Hence, the combination of FA and muscle exercise effectively improved motor function and increased HSP-70 expression and TH-positive fibers in the corpus ST in rotenone-induced PD mice [172].

Sirtuin 2 (SIRT2) is a potential culprit in PD pathology and modulates the $\alpha$-synuclein accumulation that is critical to several pathological processes in PD. Recent in vitro and in vivo studies using PD models have revealed that pharmacological inhibition of SIRT2 activity potentially ameliorates the $\alpha$-synuclein-mediated toxicity reported previously [236]. Deacetylation of Nrf2 by SIRT2 leads to a reduction in both nuclear and total cellular levels of Nrf2 through its degradation [237]. Interestingly, FA treatment prevents MPTP-induced oxidative stress through activation of ERK1/2 signaling and inhibition of SIRT2, processes that are facilitated by independent mechanisms. Additionally, FA attenuated motor impairments in MPTP-injected $\alpha$-synuclein knockout mice and wild-type mice, but not in Nrf2 knockout mice [173]. Therefore, these antioxidant and anti-inflammatory properties add to the value of FA as a therapy for PD.

6.5. Neuroprotective Mechanisms of Thymoquinone in PD. Thymoquinone (TQ) is a pharmacologically active com- pound found in black cumin seeds and plants from the Lamiaceae family [238]. Black cumin has been used in medicine since ancient times; more recently, interest in this compound has increased significantly [239]. Several investigations have demonstrated that black cumin seeds and their active constituent, TQ, may be suitable for clinical trials because most of the major effects of TQ have been shown to be beneficial. Intake of any black cumin seed has a recommended daily intake range of approximately $250-1000 \mathrm{mg}$ [240]. Previous experimental studies have confirmed that TQ and its derivatives evince several pharmaceutical activities, including antioxidant, anti-inflammatory, antihypertensive, antiasthmatic, antidiabetic, and antitumor properties [241-243]. Interestingly, the effects of TQ were studied for the $\alpha$-synuclein-induced synaptic toxicity in the cultures of rat hippocampal neurons and neurons differentiated from human induced pluripotent stem cells (IPSCs). In both types of cultures, TQ protected the neurons against $\alpha$-synucleininduced synaptic damage, increased the level of synaptophysin (a synaptic density marker), and prevented inhibition of synaptic vesicle recycling induced by the mutant $\beta$-synuclein $(\mathrm{P} 123 \mathrm{H})$. Moreover, using cells cultured on the multielectrode arrays, the authors demonstrated that TQ maintained normal bioelectrical activity in the neuronal network that was damaged by the actions of $\alpha$-synuclein [244]. As previously described, autophagy is a natural cellular mechanism for eliminating unnecessary or damaged organelles and molecules and can be induced by oxidative or toxic stress. The disturbance of the autophagy mechanism can lead to the development of neurodegenerative diseases. TQ at a concentration of $0.0110 \mu \mathrm{M}$ prevented the $\mathrm{MPP}^{+}$-induced death of mesencephalic dopaminergic neurons in vitro by reducing the release of lactate dehydrogenase and maintaining MMP. The effect of TQ was accompanied by the activation of autophagy, which contributed to the reduction of the apoptotic neuron death [245].

The neuroprotective effect of TQ was demonstrated in a rotenone-induced PD model, in which TQ treatment prevented the death of primary dopaminergic neurons [246]. Furthermore, TQ significantly upregulated the expression of neuroprotective proteins, significantly downregulated the expression of proinflammatory cytokines, and inhibited activation of NF- $\kappa$ B against LPS/IFN $\gamma$-activated BV- 2 microglial cells [247]. Importantly, TQ manifested the ability to improve the course of PD in in vivo experiments. In mice with MPTPinduced PD accompanied by the development of oxidative stress and neuroinflammation in the brain, TQ restored the activities of anti-inflammatory enzymes, prevented GSH exhaustion, inhibited lipid peroxidation, and decreased levels of proinflammatory cytokines [174]. In a rotenone-induced PD model, TQ prevented the development of motor impairments and changes in the content of Parkin and Drp1 proteins and increased DA levels in the SN and ST areas of rat brain [175]. The neuroprotective action of TQ was observed in the animals after injection of 6-OHDA into the ST which led to the loss of neurons and behavioral functions [176].

6.6. Neuroprotective Mechanisms of Ellagic Acid in PD. Ellagic acid (EA) is a natural antioxidant phenol found in several 
vegetables and fruits, in particularly large quantities in persimmon, pomegranates, nuts, black raspberry, raspberry, peach, strawberry, and plumes [248]. There is a relatively high content of EA in raspberries $(1500 \mathrm{mg} / \mathrm{g}$ dry weight), strawberries $(630 \mathrm{mg} / \mathrm{g}$ dry weight), cranberries $(120 \mathrm{mg} / \mathrm{g}$ dry weight), walnuts (590 mg/g dry weight), pecans (330 mg/ g dry weight), and a number of other plant foods [249]. The daily intake of total polyphenols is the highest in Denmark at approximately $1786 \mathrm{mg} /$ day, followed by Japan at $1500 \mathrm{mg} /$ day. European countries and the Americas average about $900 \mathrm{mg} /$ day and $800 \mathrm{mg} /$ day, respectively. In Europe, Poland and France have the highest intake-after Denmark-at above $1000 \mathrm{mg} /$ day; Italy averages approximately $650 \mathrm{mg} /$ day, Greece about $584 \mathrm{mg} /$ day, and Spain has the lowest intake at $300 \mathrm{mg} /$ day [250]. In Asian countries, such as China and Korea, apples and vegetables seem to serve as the main sources of polyphenols, while green tea provides the highest intake for the Japanese population [251]. The appropriate dose of an EA supplement depends on several factors, including the age and health of the consumer. Currently, there are no standard dosing recommendations for EA.

Moreover, EA has been shown to have potentially antioxidant, anti-inflammatory, antiviral, antisteatotic, antibacterial, antihepatotoxic, anticholestatic, anticancer, antidiabetic, immunomodulatory, and antiproliferative properties, representing an extensive range of beneficial effects that may be applied to improve human health [248, 252-254]. The bioavailability of EA is low compared with that of other phenols; however, punicalagin, which is a source of EA, was detected in human plasma after consumption of pomegranate juice $[255,256]$. Evidence indicates that pomegranate juice containing $318 \mathrm{mg}$ of punicalagin and $25 \mathrm{mg}$ of EA consumption led to a plasma concentration of $31-33 \mathrm{ng} / \mathrm{mL}$ (maximum concentration) EA $1 \mathrm{~h}$ after absorption $[255,256]$. After oral administration to rats, both punicalagin and EA were detected in the plasma after consumption of pomegranate leaf extract [257-259].

Several preclinical trials have suggested that EA effectively attenuates renal oxidative damage in LPS/D-galactosamine-induced PD rats by reducing the levels of lipid peroxidation and boosting the antioxidative defense system via increased levels of enzymic and nonenzymic antioxidant activities [260]. Moreover, treatment with EA enhanced the GSH content and mRNA expression of renal and hepatic SOD and GPx activity. In addition, EA treatment also prevented elevated renal and hepatic levels of MDA and NO production [261]. Of note, EA administration prevented 6OHDA-induced increased levels of MDA and decreased GPx and SOD activities in both ST and hippocampus tissues. EA treatment also improved motor function and electrophysiological performance in medial forebrain bundlelesioned rats by raising cerebral antioxidant content [177]. Other studies discovered that combined treatment with three compounds such as EA, $\alpha$-lipoic acid, and myrtenal attenuated decreases in DA levels, substantially decreased lipid peroxidation levels, and restored CAT activity in 6-OHDAinduced PD rats [262].

Interestingly, a study involving the kinetics of enzyme inhibition demonstrated that EA combined with curcumin inhibited MAO-B activity via both competitive and noncompetitive inhibition [263]. Importantly, previous research has shown that dimethyl fumarate, a pharmacological activator of Nrf2 that is presently used in multiple sclerosis, may also exert beneficial effects in PD [264]. EA was also utilized to confer neuroprotection against rotenone-induced neurotoxicity through activation of $\mathrm{Nrf} 2$ signaling, which was involved in EA-mediated DA neuroprotection [265]. Furthermore, similar research also revealed that EA reduced striatal MDA levels, ROS, and DNA fragmentation and improved Nrf2, HO-1, and behavioral functions. Meanwhile, EA prevented the loss of TH-positive neurons within the SN in 6-OHDA-induced rat models of PD [178]. These results suggest EA is a potential Nrf2 activator, and that by restoring antioxidant mechanisms, EA may serve as a promising therapeutic candidate for PD in the future.

6.7. Neuroprotective Mechanisms of Caffeic Acid in PD. Caffeic acid (CA) is a phenolic acid extracted from numerous plant species and is present in beverages such as coffee, wine, and tea. CA carries medicinal properties such as those found in propolis, which has been studied extensively for its strong antioxidant potential [266-269]. Emerging epidemiological evidence suggests that greater coffee consumption may reduce the risk of NDDs. The daily intake of CA has been estimated to be about 500-1000 mg in humans consuming fruits, vegetables, beer, and coffee [226]. Drinking a single cup of coffee provides as much as 70-350 mg of chlorogenic acid or CA [270]. Recent evidence revealed the highest category of coffee consumption (Netherlands, 6 cups/day; Sweden, 2-5 cups/day; Spain, 1-2 cups/day; Japan, 3-5 cups/day; Italy, 1-3 cups/day; Finland, 1-4 cups/day; and China, 1-3 cups/day) [33, 271].

CA has been reported to have several pharmacological activities, including anticancer, anti-inflammatory, and neuroprotective properties $[180,272]$. It has also been reported that CA may react with peroxyl radicals involved in lipid peroxidation to efficiently mitigate several disease conditions of $\mathrm{PD}[180,273]$. CA is believed to remove excess ROS/RNS generation, and $\mathrm{CA}$ is known to stimulate antioxidative enzyme activities including SOD, GPx, GSH, and CAT [273]. In PQ-induced fruit fly, using a Drosophila melanogaster model, a molecular docking study demonstrated the strong interaction of CA with Drosophila melanogaster transcriptional activation of $\mathrm{Nrf} 2$. In observations of the binding of CA to the Keap1 domain of Nrf2, results show that the protective effect of CA reduction of lipid peroxidation level and ROS in Drosophila melanogaster was possible through its coordination, which delays Nrf2-Keap1 binding and leads to an enhanced antioxidant defense system [179]. In the 6OHDA-induced SH-SY5Y cellular model, CA-phenethyl ester significantly improved cell viability, diminished apoptotic cell death, and prevented changes in damaged nuclear morphology. Furthermore, treatment with CA-phenethyl ester could maintain mitochondrial function, inhibit ROS production, upregulate Bcl-2 and Akt levels, and downregulate Bax expression [274]. Similarly, in $\mathrm{MPP}^{+}$-induced neurotoxicity in vitro $\mathrm{PD}$ models, CA-phenethyl ester directly inhibited release of Cyto-C and apoptosis-inducing factor (AIF) from mitochondria [275]. 
Furthermore, one highlight resulting from CA administration was a significant increase in the efficiency of striatal DA content and, consequently, a decrease in microglia expressions and inflammatory mediators. In addition, histopathological assessment of $\mathrm{SN}$ neurons demonstrated high immunostaining for TH-positive cells and improved motor performance in rotenone-treated mice [276]. In sevenmonth-old A53T $\alpha$-synuclein transgenic mice, CA alleviated cell damage and reduced A53T $\alpha$-synuclein expression by activating the JNK/Bcl-2-mediated autophagy signaling pathway. It was also observed that the CA's protective efficacy on A53T $\alpha$-synuclein degradation was prevented by the JNK inhibitor SP600125 and the autophagy inhibitor bafilomycin A1. In A53T Tg mice, CA attenuated loss of dopaminergic neurons, improved behavioral function, enhanced autophagy activation, and reduced $\alpha$-synuclein accumulation in the SN [180]. In MPTP-induced PD mice, CA lowered the production of proinflammatory cytokines, diminished the expression of inflammatory mediators and GFAP, and decreased the production of $\mathrm{NO}$ and $\mathrm{PGE}_{2}$. In addition, $\mathrm{CA}$ restored the expression of BDNF and GDNF and maintained TH-positive cells and DA synthesis [181]. Previous research discovered that metallothionein- (MT-) 1 and MT-2 were upregulated, particularly in ST astrocytes, by activation of Nrf2 signaling in response to oxidative stress and acted to protect SN neurons [277]. Treatment with CA protected both $\mathrm{SN}$ and intestinal enteric neurons and upregulated MT-1 and MT-2 antioxidative molecules in the ST astrocytes of rotenone-induced $\mathrm{C} 57 \mathrm{BL} / 6 \mathrm{~J}$ mice [278]. Interestingly, the inhibitory effect of CA against escitalopram-induced $\alpha$-synuclein accumulation and other neurotoxins may prove to be promising novel therapeutic drugs for PD [279].

6.8. Neuroprotective Mechanisms of Epigallocatechin-3-Gallate in PD. Epigallocatechin-3-gallate (EGCG), a polyphenol isolated from green tea, is known for its myriad physiological beneficial effects against inflammatory disorders, cancer, and NDDs in humans [280-282]. Several pharmacological activities including antioxidant, anti-inflammatory, metalchelating, radical-scavenging, antiapoptotic, and anticarcinogenic properties by modulation of several transcription factors, proteins, and other important growth factors are attributed to EGCG [282, 283]. EGCG's broad potential for improving healthy aging derives from its promotion of morphologic and functional alterations that occur naturally in an aging brain; these alterations increase memory and learning ability, suppress cognitive dysfunction, and reduce oxidative damage in the brain [284-286]. The typical daily intake of EGCG resulting from the consumption of green tea infusions ranges from 90 to $300 \mathrm{mg} / \mathrm{day}$, while exposure in high-level consumers is estimated to be as much as $866 \mathrm{mg}$ EGCG/day in the adult population in Europe. Food supplements containing green tea catechins provide a daily dose of EGCG in the range of 5-1000 $\mathrm{mg} /$ day for the adult population. Based on available data on the potential adverse effects of green tea catechins on the liver, the evidence from interventional clinical trials of doses equal to or above $800 \mathrm{mg} /$ day in the form of a food supplement has shown increased bioavailability of EGCG in treated subjects compared to control
[287-290]. A few studies have previously demonstrated that EGCG can cross the BBB easily, which is crucial for the development of therapeutic agents for PD [291, 292].

Several studies of human subjects have described an inverse dose-response relationship between green tea consumption and cognitive impairments in PD disorders [293, 294]. Recent advancements in case-controlled joint studies revealed that daily consumption of two or more cups of tea reduced cognitive dysfunction and decreased the prevalence of PD [295, 296]. A Finnish longitudinal study conducted over 13 years with 30000 adults aged $25-74$ years old showed that daily consumption of three or more cups of tea reduced the risk of PD [297]. Similarly, a large-scale-cohort, 20-year follow-up study involving nearly 50000 male and 80000 female volunteers demonstrated that EGCG intake was associated with a $40 \%$ lower risk of PD [298]. Moreover, several cohort- and case-controlled studies across Asian, European, and North American countries have reported a link between tea consumption and lower risk of PD [299]. In Asian populations, particularly among the Chinese, $28 \%$ of populations experienced a decreased risk of developing PD with daily consumption of three cups of tea for ten years [299].

Currently, several findings have demonstrated the antioxidative capacity of EGCG under experimental conditions in both cellular and animal models $[300,301]$. In rotenoneinduced rat PD models, EGCG treatment reduced lipid peroxidation production and $\mathrm{NO}$ levels, increased succinate dehydrogenase (SDH) activity, improved mitochondrial function, and raised ATPase and ST catecholamine levels. In addition, EGCG treatment decreased the level of neuroinflammatory cytokines and apoptotic markers and improved motor performance [302]. Administration of EGCG in MPTP-induced mice prevented neurotoxin-induced reduction in ST antioxidant enzymes SOD and CAT and increased the activities of both enzymes in the total brain [303]. Furthermore, EGCG treatment wholly prevented STAT3 activity and stimulated neuronal cell proliferation induced by 6 OHDA in SH-SY5Y cells [304]. Most recent findings have confirmed that the neurorescue effect of EGCG regulated the iron-export protein ferroportin in the $\mathrm{SN}$, reduced oxidative stress, and attenuated functional and neurochemical shortages against MPTP-induced PD mice [182]. In the MPTP-injected mouse model, EGCG restored movement behavior and protected $\mathrm{TH}$-positive cells in the $\mathrm{SN}$ region. Flow cytometric analysis showed that the ratio of $\mathrm{CD} 3_{+} \mathrm{CD} 4_{+}$ to $\mathrm{CD} 3_{+} \mathrm{CD} 8_{+} \mathrm{T}$-cell lymphocytes in the peripheral blood increased with EGCG treatment and reduced expression of inflammatory factors such as TNF- $\alpha$ and IL- 6 in the serum [183]. Furthermore, EGCG was shown to display antiapoptotic effects in PQ-induced PC12 cell models: EGCG maintained MMP and inhibited the upregulation of caspase-3 activity and the downregulation of the proapoptotic SMAC protein in cytosol expression [305]. It was also observed that EGCG treatment reduced TNF- $\alpha$ and NO inflammatory mediators and attenuated loss of midbrain DA levels triggered by LPS-induced neurotoxicity [306]. Furthermore, in vitro and in vivo studies demonstrated that cotreatment with EGCG lowered glutamate-induced oxidative cytotoxicity in HT22 cells through inhibition of NF- $\kappa$ B activation. In 
addition, EGCG treatment moderated the effect of decreased accumulation of 3-O-methyldopa in the plasma and ST of rats that were administered carbidopa+L-Dopa; EGCG also exerted a strong therapeutic effect against kainic acidinduced oxidative neuronal death in the hippocampus of $\mathrm{PD}$ rats [307].

The process of $\alpha$-synuclein and other protein deposition has been strongly linked with numerous NDDs, including PD. Recently, several studies have attracted attention by showing that $\alpha$-synuclein accumulation formation might be mediated by small molecules, such as the polyphenol EGCG, which may offer a potential therapeutic option for management of $\alpha$-synucleinopathies [308, 309]. Other research found that EGCG potentially interacted with $\alpha$-synuclein amino acid sites found on peptide membranes. It was implicated that EGCG binds to $\alpha$-synuclein via unstable hydrophobic interactions; these findings support the assertion that EGCG could be a potent remodeling agent of $\alpha$-synuclein accumulation and a potential disease-modifying agent for the treatment of PD [184].

6.9. Neuroprotective Mechanisms of $\alpha$ - and $\beta$-Asarone in PD. Alpha- $(\alpha-)$ asarone and beta- $\left(\beta_{-}\right)$asarone compose an important antioxidant aromatic chemical constituent that is extracted from the rhizomes of Acorus calamus. Consequently, both $\alpha$ - and $\beta$-asarone have been reported to have one or more similar pharmacological properties that may offer beneficial effects in the therapeutic management of several diseases [310-313]. Importantly, the delivery of $\alpha$ - and $\beta$-asarone in the brain is extensive, demonstrating its ability to cross the $\mathrm{BBB}$, a desirable characteristic of compounds used for the treatment of numerous NDDs [310]. A recent Swiss ADME predictor study revealed that absorption, distribution, metabolism, and excretion results showed that $\alpha$ - and $\beta$-asarone possess good oral bioavailability; the study also showed a good binding affinity towards dopaminergic receptors. Further, $\alpha$ - and $\beta$-asarone were found to interact with different amino acid residues of disease-modifying D2 and D3 receptors through hydrogen bonding [314].

In one PD model, $\alpha$-asarone treatment reduced neural inflammation and suppressed IL- $\beta$, IL- 6 , and TNF- $\alpha$ production in LPS-stimulated BV- 2 cells. In addition, $\alpha$-asarone treatment effectively inhibited the LPS-stimulated activation via regulation of NF- $\kappa$ B by blocking degradation of inhibitor $\mathrm{NF}-\kappa \mathrm{B}$ signaling in BV-2 microglial cells. In vivo studies also demonstrated that prophylactic administration with $\alpha$-asarone inhibited microglial activation and attenuated PD-like behavioral deficits in MPTP-injected PD mice [185]. In the 6-OHDA-induced PD model, $\beta$-asarone improved the behavioral function of rats in the initiation time, open field, stepping time, and rotarod tests. Research has also found that $\beta$-asarone increases the levels of HVA, DOPAC, and 5-HIAA in the ST region. In addition, administration with $\beta$-asarone elevated the level of TH-positive neurons and inhibited the expression of LC3-II in SN4741 cells. Moreover, in vivo experimental results showed that $\beta$-asarone affected the expression of Bcl-2, Beclin-1, JNK, and p-JNK in 6-OHDAinjected PD rats. The neuroprotective effect of $\beta$-asarone occurs primarily by downregulating JNK and p-JNK expres- sions and then indirectly increasing Bcl-2 expression. Additionally, $\beta$-asarone may inhibit the function of Beclin-1, thereby inhibiting autophagy activation [186]. Activated autophagy is an important process that may play a defensive role through clearance of toxic aggregated $\alpha$-synuclein in neurons [315]. On the other hand, dysfunction of the autophagy-lysosomal pathway has been associated with the development of PD [316]. Recent studies also proposed that endoplasmic reticulum (ER) stress may induce autophagy [317]. In 6-OHDA-induced PD rat models, $\beta$-asarone administration may decrease the levels of Beclin-1, CHOP, GRP78, and p-PERK while significantly increasing the level of $\mathrm{Bcl}-2$. $\beta$-Asarone may increase $\mathrm{Bcl}-2$ by inhibiting the $\mathrm{p}$ ERK pathway, and Bcl-2 may inhibit the expression of Beclin-1. The results of that study suggested that $\beta$-asarone may regulate autophagy and ER stress via the PERK/$\mathrm{CHOP} / \mathrm{Bcl}-2 / \mathrm{Beclin}-1$ pathway [187]. Very recent findings have shown that $\beta$-asarone can effectively inhibit neuronal apoptosis through the CaMKII/CREB/Bcl-2 signaling pathway and regulate $\mathrm{Bcl}-2$ family proteins [318]. Moreover, $\beta$ asarone significantly lowered the expression levels of MALAT1 and $\alpha$-synuclein in the midbrain of MPTPinjected $\mathrm{PD}$ mice. In addition, immunoprecipitation and RNA pull-down assays confirmed that MALAT1 was associated with $\alpha$-synuclein, leading to the increased stability of $\alpha$ synuclein and its expression in SH-SY5Y cells. $\beta$-Asarone treatment could increase the viability of cells exposed to $\mathrm{MPP}^{+}[319]$. Similarly, another study demonstrated that $\beta$ asarone exerted antioxidative effects on $\mathrm{H}_{2} \mathrm{O}_{2}$-stimulated PC12 cells by reducing oxidative stress via activation of the protective Nrf2/HO-1 pathway [320].

Based on the pharmacological effect of $\beta$-asarone, previous scientific studies demonstrated that combined treatment with $\beta$-asarone and L-dopa carried potentially therapeutic value. Interestingly, combined treatment with $\beta$-asarone and L-dopa reduced the level of creatinine and increased the level of HVA, DA, DOPAC, and 5-HT, while $\beta$-asarone also enhanced TH and DAT protein expression in madoparinduced PD rats [321]. Similar findings also show that LC3B and Beclin-1 expression decreased, while p62 expression increased after coadministration with $\beta$-asarone and Ldopa. In addition, the group that received coadministered $\beta$-asarone and L-dopa exhibited a significant decrease in autophagosome activity when compared with the 6OHDA-injected PD control group [322]. However, further experimental validation using in vitro and in vivo studies is needed before clinical trials may commence.

6.10. Neuroprotective Mechanisms of Theaflavin in PD. Theaflavin (TF) is representative of a group of polyphenols that are found in black tea, comprising theaflavin-3-gallate, theaflavin- $3^{\prime}$-gallate, and theaflavin- $3,3^{\prime}$-digallate, which contribute to the quality and color of black tea [323]. TF is known for its several therapeutic effects owing to its antioxidant properties: removal of excess free-radical formation and metal chelation ability [324-327]. In the past few years, several scientific reports have shown that TF has potential neuroprotective effects against NDDs. TF has been found equal in efficiency to EGCG at inhibiting $\beta$-amyloid- and 
$\alpha$-synuclein-induced neurotoxicity due to its potential antioxidant properties [328]. Recently, a randomized, doubleblind, placebo-controlled study showed that the beneficial TF doses of $50 \mathrm{mg} /$ day and $100 \mathrm{mg} /$ day provide health benefits. These TF doses also appeared to be more effective than similar doses of tea catechin [329]. Recently, a clinical survey from the European Union demonstrated that the daily intake of TF ranged from $181 \mathrm{mg} /$ day (Czech Republic) to $793 \mathrm{mg} /$ day (Ireland). The highest intakes of TF were observed in Ireland (191-505 mg/day) and the lowest intakes in Spain (9-24 mg/day) [330]. Little data exists on the pharmacokinetic profile of TF in humans: after the consumption of $700 \mathrm{mg}$ of TF once a day, corresponding to about 30 cups of black tea, achieved maximum concentration observed in blood plasma and betterment of oral bioavailability [331].

Cell lines were used to investigate the mechanism of action of TF, 6-OHDA-induced SH-SY5Y, and the findings revealed attenuated loss of cell viability, reduced nuclear morphology, decreased apoptosis, increased MMPs, and diminished intracellular NO levels. These results suggested that TF had a protective effect against 6-OHDA-induced apoptosis through inhibition of $\mathrm{NO}$ and ROS production [332]. In the PC12 cell line treated with $\mathrm{H}_{2} \mathrm{O}_{2}$, oxidative stress was eliminated by administration of TF, which decreased Bax and caspase- 3 protein expression and increased Bcl-2 expression. This finding indicates that TF possesses antiapoptotic properties, providing both cytoprotection and neuroprotection [333]. Another study demonstrated that TF acted as a potent inhibitor of $\beta$-amyloid and $\alpha$-synuclein fibrillogenesis and stimulated the $\beta$-amyloid and $\alpha$-synuclein assembly into a nontoxic form. These results suggest that TF could be used to remove toxic amyloid deposits [328].

In the PD mouse model, TF increased the expression of DAT and VMAT-2 in addition to downregulating the effects of oxidative stress in MPTP-induced neurotoxicity in mice. TF has also been shown to ameliorate dopaminergic neuronal loss and behavioral deficits [188]. In mice treated with $\mathrm{MPTP} / \mathrm{p}$, TF administration increased the expression of nigral TH and DAT and lowered caspase-3, caspase-8, and caspase-9; these results were accompanied by increases in regulated behavioral function [189]. Moreover, dysfunction of the cholinergic system was also observed to trigger the production of proinflammatory cytokines and activation of microglia: the levels of IL- 4 and IL-10 anti-inflammatory markers were raised in MPTP-injected mice as a compensatory mechanism against neuronal inflammation [334]. In a recent in vivo study, treatment with TF ameliorated the chronic MPTP-induced neurotoxicity in the SN and ST of mice, as evidenced by significantly decreased neuroinflammation and apoptosis. In addition, treatment with TF attenuated the MPTP-injected behavioral impairments such as catalepsy and akinesia and significantly reduced the excess formation of IL-4 and IL-10 anti-inflammatory cytokines [190].

\section{Neuroprotective Roles of Antioxidant Phytochemicals and Their Analogs}

As the present study has explained, some naturally derived phytochemical constituents are potential neuroprotective agents that may have applications for treating PD. The chrysin derivatives 6,8-bis(o-tolylselanyl)-chrysin, 6,8-bis ( $p$-anisoylselanyl)-chrysin, and 6,8-bis( $p$-fluorophenylselanyl)-chrysin were semisynthesized and studied for their antioxidant and neuroprotective activity [335]. The author of that study suggested that the structure-activity relationship, with all three compounds containing a fluorine atom in the para position to selenium, showed the greatest antioxidant activity through its ability to inhibit lipid peroxidation and ROS generation in mouse cortex and hippocampus. Scipioni et al. [336] demonstrated that synthesized novel vanillin derivatives of $4,4^{\prime}$ - $(((3$-hydroxypropyl)azanediyl)bis(methylene))bis(2-methoxyphenol) and $4,4^{\prime}, 4^{\prime \prime}$, $4^{\prime \prime \prime}-((1,4-$ phenylenebis(azanetriyl))tetrakis(methylene))tetrakis(2-methoxyphenol) having a tertiary amino group, accompanied by the number of vanillin moieties, have confirmed ability to protect from oxidative damage and potential antioxidant activity in $\mathrm{H}_{2} \mathrm{O}_{2}$-induced neuroblastoma SH-SY5Y cells. Another study reported that semisynthesized AA derivatives including AS-2, AS-2-9-006, and AS-9-006 exhibited the greatest activity in the active avoidance, passive avoidance, and the Morris water maze tests and cognitive-enhancing activity [337]. 1-Feruloyl glycerol (FA-G1) and 1-feruloyl diglycerol (FA-DG1), two water-soluble derivatives of FA, exhibited neuroprotective effects against $\beta$-amyloid-induced neurodegeneration in both in vitro and in vivo experiments. This neuroprotection was evidenced by inhibition of NO production and reduction in iNOS expression in a dosedependent manner mediated by suppression of NF- $\kappa$ B nuclear translocation in primary astrocytes, by beneficial effects against abnormal activation of astrocytes, and by a reduction in neurodegeneration [338].

A very recent study demonstrated that lipophilic butyl ferulate, a derivative of FA, binds to amide $\mathrm{NH}$ in $\mathrm{G} \ln 15$ and Lys 16 via a hydrogen bond. This binding significantly attenuated intracellular ROS formation and could potentially upregulate antioxidant enzyme activity by modulating the Keap1-Nrf2-ARE signaling pathway [339]. Another study reported the neuroprotective effect of two CA derivatives known as caffeic acid phenethyl ester and danshensu ((R)-4 2-hydroxy-3-(3,4-dihydroxyphenyl)propionic acid), which contain numbers of hydroxyl groups in an aromatic ring (A) that are conjugated with double bond. These study results demonstrated that these two compounds significantly boost the endogenous antioxidant defense system and modulation of the PKA/CREB signaling pathway. Furthermore, these compounds significantly improved behavioral performance in both the step-down avoidance test and Morris water maze test [340]. A similar study also revealed that CAphenethyl ester 4-O-glucoside, which is synthesized from CA, suppressed $\mathrm{H}_{2} \mathrm{O}_{2}$-induced oxidative stress by inhibiting ROS generation, protein carbonylation, and MDA content, in addition to significantly enhancing GSH and SOD activities in both SH-SY5Y and PC12 cells. Mechanistically, it prevented impairments in learning and cognition in vivo by reducing neuronal cell death and protecting against hippocampus and cortex dysfunction [341]. The most promising polyphenolic TF derivatives, namely, TF-3-gallate, TF-3' gallate, and TF- $3,3^{\prime}$-gallate, exhibited significant rescue from 
the metabolic inhibition induced by $\alpha$-synuclein aggregates in PC12 cells [328].

\section{Neuroprotective Role of Phytochemical Nanoformulation}

Several nanodelivery systems loaded with naturally derived antioxidant phytochemicals have been demonstrated to be effective in modulating oxidative stress, $\alpha$-synuclein aggregation, chronic inflammation, and various signaling pathways that mediate most aging-associated NDDs, particularly in PD. For instance, in a study by Giacomeli et al. [342], chrysin-loaded lipid-core nanocapsules showed higher antioxidant ability and reduced neurotoxicity through reduction of oxidative stress and neuroinflammation and through modulation of neurochemical and behavioral changes in an aged animal, compared with free chrysin. A very recent study observed that chrysin-loaded poly (lactic-co-glycolic acid) nanoparticles less than $150 \mathrm{~nm}$ in size in pentylenetetrazolinduced epilepsy mice showed chrysin nanoparticle treatment counteracted oxidative stress, reduced neuronal apoptosis, and upregulated $\mathrm{Nrf} 2, \mathrm{HO}-1$, and $\mathrm{NAD}(\mathrm{P}) \mathrm{H}$ quinone oxidoreductase [343]. Trans-FA-loaded solid lipid nanoparticles could enable the uptake of FA by cells due to of their lipophilic nature, thereby increasing FA bioavailability and concentration-dependent reduction of lipid peroxidation and enhancement of antioxidant enzyme activities in rat brain [344]. Anti-inflammatory effects of FA-loaded nanoparticles such as modified glycol chitosan nanoparticles have also been reported previously [345]. Another study demonstrated that rats that received intravenous injections of an FA-loaded nanostructured lipid carrier exhibited significantly attenuated neurobehavioral deficits, oxidative stress, and cellular damage; the study showed that activating the PI3K pathway may be of beneficial effect in cerebral stroke [346].

There is evidence that nanoencapsulated TQ carry greater antioxidant and neuroprotective properties. In a rat model, TQ-loaded mesoporous silica nanoparticles $90 \mathrm{~nm}$ in size and spherical in shape were able to cross the BBB. Results showed that the encapsulated TQ-loaded mesoporous silica nanoparticles enhanced drug target delivery to all brain areas (ST, cortex, thalamus, midbrain, and hypothalamus) and significantly reduced oxidative stress biomarkers [347]. Numerous studies have reported that different nanoparticle-loaded phytochemicals (e.g., vitamin E, resveratrol, curcumin, and hyaluronic acid) with an average particle size of $100 \mathrm{~nm}$ resulted in higher ROS scavenging efficiency and lower lipid peroxidation in patients with PD [348-350]. Similarly, TQ-loaded PLGA-chitosan nanoparticles (particle size from $183 \mathrm{~nm}$ ) delivered via the nose-tobrain route in rodents improved their pharmacokinetic profile in the brain and enhanced grip strength and locomotor activity. In addition, these effects were supported by a significant reduction in levels of lipid peroxidation and increase in antioxidant enzyme activity in the brain of middle cerebral artery-occluded rats [351]. The anti-inflammatory and neuroprotective potential of stress-induced TQ-loaded solid lipid nanoparticles has been demonstrated by significantly enhanced antidepressive-like behavioral function, hippo- campus BDNF levels, and reduced levels of hippocampal IL-6 and TNF- $\alpha$ expression compared with free TQ [352]. TQ-loaded solid lipid nanoparticle treatment also attenuated the overexpression of GFAP, proinflammatory cytokines, and p-p 65 NF- $\kappa \mathrm{B}$ nuclear translocation; improved the number of TH-positive neurons; and ameliorated motor deficits in neurotoxin-intoxicated animals when compared with the free TQ-treated group [353].

Recently, El-Missiry et al. [354] studied the neuroprotective and antiepileptic efficacy of EA-loaded calciumalginate nanoparticles (sized approximately $150 \mathrm{~nm}$ ) in pentylenetetrazol-induced seizures in male mice. These EAloaded calcium-alginate nanoparticles were also able to ameliorate oxidative stress, as evidenced by enhanced antioxidant efficiency and decreased 4HNE levels in the brain. Furthermore, the nanoformulation outperformed free EA in several activities: amelioration of apoptosis, inhibition of Cyto-C release, activation of caspases, regulation of P53, Bax, and Bcl-2 protein expression, and protection against DNA damage. Moreover, the EA and chitosan-coated combination mitigated rotenone-induced ROS overproduction and reduced cytotoxicity [355]. The antioxidant and antiaging properties of CA-loaded nanotransfersomes were found to enhance cell viability, reduce intracellular ROS generation, attenuate lipid peroxidation, and modulate MMP expression [350]. In addition, anticonvulsive and neuroprotective effects of EGCG-loaded PEGylated-PLGA nanoparticles (particle size from $169 \mathrm{~nm}$ ) safe for brain cells significantly reduced neuroinflammatory marker expression and were capable of increasing drug integrity and bioavailability [356]. The $\alpha$-asarone-loaded lactoferrin-modified mPEGPLA nanoparticles delivered intranasally to the brain showed increased nasal permeability, brain targeting, and brain systemic exposure and reduced toxicity without affecting bioavailability [357].

\section{Conclusion}

In recent years, research into naturally derived phytochemicals targeting several pathogenic conditions of age-associated NDDs has increased, and clinically, no serious adverse reactions of currently available phytochemicals have been documented. Moreover, PD has been therapeutically cured by natural products, in particular by naturally food-derived phytochemicals with antioxidant potential that may present a reliable source of medicine. The clinical evidence of the health benefits of phytochemicals is not yet fully accepted. However, naturally derived lipophilic phytochemicals can enter the brain and cross the BBB easily, offering increased bioavailability, faster metabolism, and higher affinity to receptors. The regular administration of these naturally derived phytochemicals is an imperative approach to enhance the reversal of neuron function decline and disease resistance competency. Much of the in vivo experimental evidence presented in the current study of the potential neuroprotective effects of antioxidant phytochemicals was supported by the results of histopathological and immunohistochemical investigation, which indicated the protection of dopaminergic neurons and attenuation of the loss of 


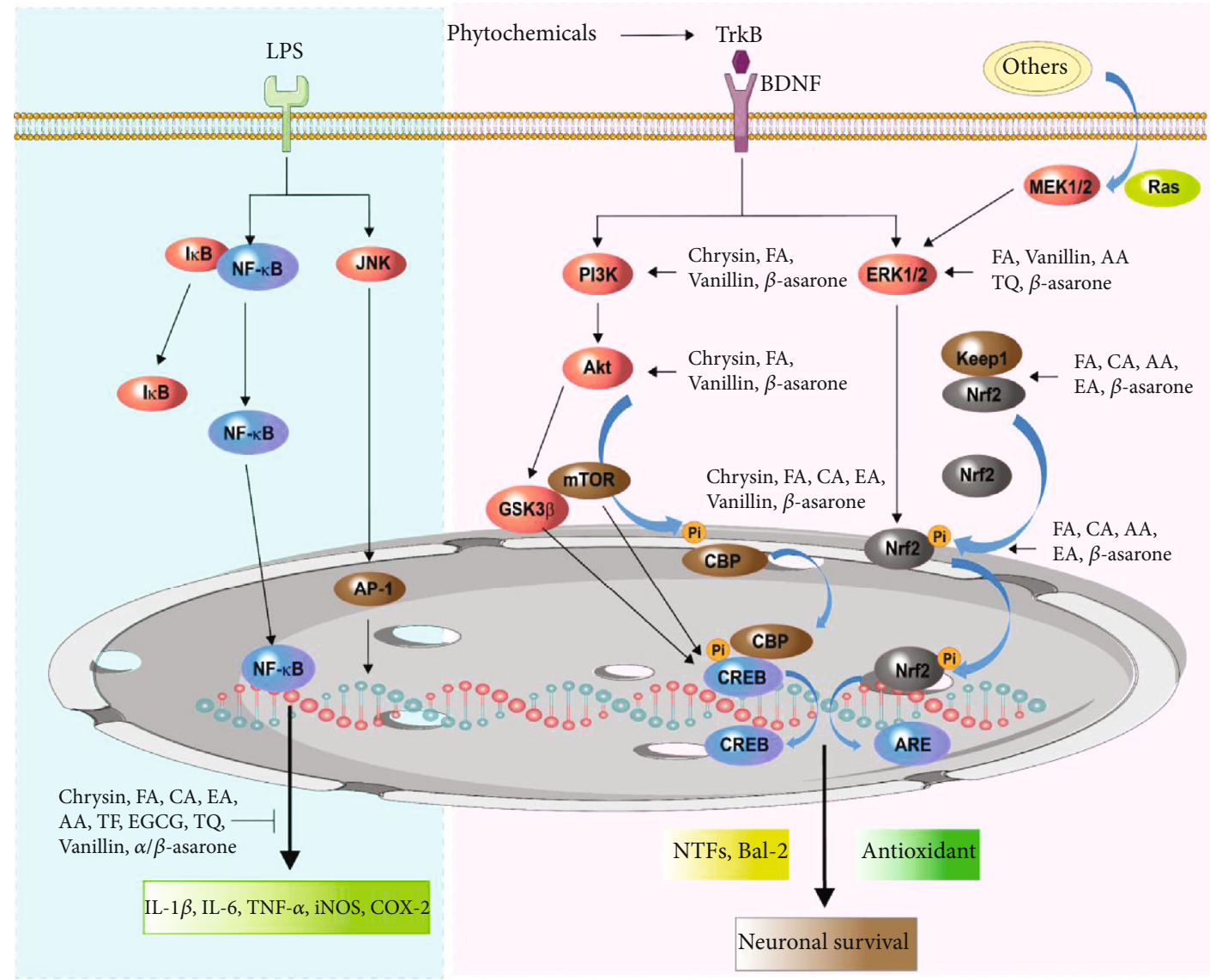

FIGURE 3: Intracellular targets of neuroprotective antioxidant phytochemicals by activation of Keap1/Nrf2/ARE signaling pathways to increase the expression of antioxidant enzymes. The modulation of these pathways by natural antioxidant phytochemicals such as chrysin, vanillin, asiatic acid (AA), ferulic acid (FA), thymoquinone (TQ), ellagic acid (EA), caffeic acid (CA), epigallocatechin-3-gallate (EGCG), $\alpha$ - and $\beta$-asarone, and theaflavin (TF).

TH-positive cells. Furthermore, these changes were accompanied by improvement of several neurotoxin-induced motor balance and cognitive deficits of study animals, as evaluated by rotational behavior, open field test, beam walking, and horizontal and vertical grid tests, as well as by passive avoidance, Barnes maze, and other nonmotor behavioral patterns. Table 1 provides a summary of well-recognized and reported antioxidant phytochemicals and their molecular mechanisms of action in PD.

Collectively, the antiparkinsonian effects of antioxidant phytochemicals have been demonstrated in the reports cited in the present study. It is known that oxidative stress and neuroinflammation are important factors responsible for the progression of PD. Hence, our study provides evidence that the anti-inflammatory activities of antioxidant phytochemicals offer a safe approach to protect against the neuronal damage by reducing oxidative stress, inhibiting lipid peroxidation activity, enhancing the content of GSH, modulating the secretion of proinflammatory cytokines, regulating inflammatory mediators such as COX-2, iNOS, and NO, and regulating anti-inflammatory molecules and pathways (Figure 3). Abundant evidence suggests that oxidative stress and $\alpha$-synuclein accumulation trigger activation of microglia and astrocytes; this activation is associated with the complex neuroinflammatory pathways leading to neurodegeneration in PD. A number of antioxidant phytochemicals have been shown to clear $\alpha$-synuclein accumulation and to inhibit microglial activation accompanied by suppressed IBA-1 and GFAP expression. Simultaneously, several antioxidant phytochemicals discussed enhanced the expression of GDNF and BDNF levels involved in the survival of DA neurons. Recently, several experimental findings demonstrated that the JNK signaling pathway involved in apoptotic actions belonging to the superfamily of MAPKs responds to induced ROS and plays a significant role in triggering apoptosis. Several antioxidant phytochemicals are being directed towards some molecular pathways underlying the neuroprotective properties; thus, phytochemicals have been observed to restore the reduced level of antiapoptotic Bcl-2, lowering the expression of proapoptotic Bax and inhibiting caspase activity. Furthermore, phytochemicals have been confirmed to reduce JNK activation and transcription factor c-Jun, resulting in the reduction of dopaminergic neuron apoptosis.

Together, oxidative stress, neuroinflammation, and Nrf2/ ARE deregulation are common major situations in the pathogenesis of NDDs, particularly in PD, resulting in impaired motor function and neuronal cell death. Moreover, some important modulators of Nrf2/ARE pathways and autophagy 
are altered in PD. Again, neuroprotection by antioxidant phytochemicals is associated with the activation of Nrf2/ARE pathways and autophagy signaling, which appear to be the most well-studied mechanisms for PD treatments. Moreover, inhibition of NF- $\kappa \mathrm{B}$ activation should be investigated further as a useful therapeutic approach to the treatment of PD. Accumulated data strongly suggest that antioxidant phytochemicals' potential for activating Nrf2/ARE pathways and autophagy signaling, shown to enhance the expression of Nrf2/ARE and autophagy-related genes, proves protective in several experimental models of PD (Figure 3).

In summary, naturally derived phytochemicals and their derivatives play a potential neuroprotective role in their multidimensional ability to regulate and modulate chronic inflammation, oxidative stress, and downstream signaling, the hallmarks of PD. In addition, to prevent the occurrence of NDDs and their threat to the population, it is essential to explore novel interventional procedures in the clinic for direct employment of dietary phytochemicals as supplements in everyday use. Moreover, the evident lack of toxicity and easy availability from natural resources highlight their advantages in adopting them in the diet. Future research should be aimed at increased clinical acceptance of claims from in vitro and in vivo preclinical studies and further clinical trial studies of several more potential compounds and the combinations thereof, to observe and prevent any undesirable side effects. The success of phytochemicals in clinical research will thereby be decisive in the evaluation of their pharmacological relevance in humans, and nutritional intervention programs will thereby decrease oxidative neuroinflammatory damage and reduce/slow down the progression of PD.

\section{Abbreviations}

AA: $\quad$ Asiatic acid

AD: Alzheimer's disease

AKT: $\quad$ Serine/threonine protein kinase

ALP: Autophagy-lysosome pathway

ARE: Antioxidant response element

Apo: Apomorphine

BBB: $\quad$ Blood-brain barrier

BDNF: Brain-derived neurotrophic factor

BSA: $\quad$ Bovine serum albumin

CAT: Catalase

COX-2: Cyclooxygenase-2

CREB: CAMP-response element binding protein

DA: Dopamine

DAT: Dopamine transporter

DBS: $\quad$ Deep brain stimulation

DOPAC: 3,4-Dihydroxyphenylacetic acid

Drp1: Dynamin-related protein 1

EGCG: Epigallocatechin-3-gallate

EA: $\quad$ Ellagic acid

Epo: $\quad$ Erythropoietin

ERK: $\quad$ Extracellular signal-regulated kinase

ER: $\quad$ Endoplasmic reticulum

FA: $\quad$ Ferulic acid

GFAP: Glial fibrillary acidic protein
GR: $\quad$ Glutathione reductase

GSH: Glutathione

GSK3 $\beta$ : Glycogen synthase kinase-3 beta

GST: $\quad$ Glutathione $\mathrm{S}$ transferases

HO-1: Heme oxygenase-1

HSP-70: Heat shock protein-70

HVA: Homovanillic acid

iNOS: Inducible nitric oxide synthase

IFN $\gamma$ : Interferon-gamma

IL-1 $\beta$ : Interleukin- $1 \beta$

IL-4: $\quad$ Interleukin-4

IL-6: Interleukin-6

IL-10: Interleukin-10

Keap1: Kelch-like ECH-associated protein

LRRK2:: $\quad$ Leucine-rich repeat kinase 2

mtDNA: Mitochondrial DNA

MAPK: Mitogen-activated protein kinase

MDA: Malondialdehyde

MPTP: 1-Methyl-4-phenyl-1,2,3,6-tetrahydropyridine

$\mathrm{MPP}^{+}: \quad$ 1-Methyl-4-phenylpyridinium

NADPH: Nicotinamide adenine dinucleotide phosphate

NDDs: Neurodegenerative disorders

NF- $\kappa$ B: $\quad$ Nuclear factor $-\kappa B$

NADPH: Nicotinamide adenine dinucleotide phosphate

NO: $\quad$ Nitric oxide

Nrf2: $\quad$ Nuclear factor erythroid 2-related factor 2

PD: $\quad$ Parkinson's disease

PGC: $\quad$ Peroxisome proliferator-activated receptor gamma coactivator

PI3K: $\quad$ Phosphoinositide 3-kinase

PINK1: $\quad$ PTEN-induced kinase 1

PKC: $\quad$ Protein kinase $\mathrm{C}$

PPAR- $\gamma$ : Peroxisome proliferator-activated receptor gamma

PQ: $\quad$ Paraquat

RNS: $\quad$ Reactive nitrogen species

ROS: $\quad$ Reactive oxygen species

SN: $\quad$ Substantia nigra

SOD: $\quad$ Superoxide dismutase

SOD2: $\quad$ Superoxide dismutase 2

ST: $\quad$ Striatum

TF: Theaflavin

TH: $\quad$ Tyrosine hydroxylase

TNF- $\alpha$ : Tumor necrosis factor-alpha

TQ: $\quad$ Thymoquinone

TrkB: $\quad$ Tropomyosin receptor kinase B

UPS: Ubiquitin proteasome system

VMAT-2: Vesicular monoamine transporter 2

6-OHDA: 6-Hydroxydopamine.

\section{Data Availability}

No data were used to support this study.

\section{Conflicts of Interest}

The authors declare no conflict of interest. 


\section{Authors' Contributions}

$\mathrm{RB}$ contributed to the design, conceptualization, organization, and writing - original draft preparation, and visualization; SA and DYC helped design and review this paper; ISK interpreted the figures; DKC was responsible for the supervision of the review and approval of the final draft. All authors have read and agreed to the published version of the manuscript.

\section{Acknowledgments}

This study was supported by "Regional Innovation Strategy (RIS)" through the National Research Foundation of Korea (NRF) funded by the Ministry of Education (MOE).

\section{References}

[1] P. A. Kempster, B. Hurwitz, and A. J. Lees, "James Parkinson's chimera: syndrome or disease?," Journal of the Royal College of Physicians of Edinburgh, vol. 47, no. 2, pp. 190195, 2017.

[2] S. Duty, "Targeting glutamate receptors to tackle the pathogenesis, clinical symptoms and levodopa-induced dyskinesia associated with Parkinson's disease," CNS Drugs, vol. 26, no. 12, pp. 1017-1032, 2012.

[3] M. F. Bastide, W. G. Meissner, B. Picconi et al., "Pathophysiology of L-dopa-induced motor and non-motor complications in Parkinson's disease," Progress in Neurobiology, vol. 132, pp. 96-168, 2015.

[4] X. S. Zeng, W. S. Geng, and J. J. Jia, "Neurotoxin-induced animal models of Parkinson disease: pathogenic mechanism and assessment," ASN Neuro, vol. 10, 2018.

[5] P. P. Michel, E. C. Hirsch, and S. Hunot, "Understanding dopaminergic cell death pathways in Parkinson disease," Neuron, vol. 90, no. 4, pp. 675-691, 2016.

[6] A. L. Fink, "The aggregation and fibrillation of $\alpha$-synuclein," Accounts of Chemical Research, vol. 39, no. 9, pp. 628-634, 2006.

[7] H. A. Lashuel, C. R. Overk, A. Oueslati, and E. Masliah, “The many faces of $\alpha$-synuclein: from structure and toxicity to therapeutic target," Nature Reviews Neuroscience, vol. 14, no. 1, pp. 38-48, 2013.

[8] S. Kaushik and A. M. Cuervo, "Proteostasis and aging," Nature Medicine, vol. 21, no. 12, pp. 1406-1415, 2015.

[9] M. Xilouri, O. R. Brekk, and L. Stefanis, " $\alpha$-Synuclein and protein degradation systems: a reciprocal relationship," Molecular neurobiology, vol. 47, no. 2, pp. 537-551, 2013.

[10] A. Chagraoui, Boulain, Juvin, Anouar, Barrière, and Deurwaerdère, "L-dopa in Parkinson's disease: looking at the "false" neurotransmitters and their meaning," International Journal of Molecular Sciences, vol. 21, no. 1, p. 294, 2020.

[11] J. Guridi and M. Alegre, "Oscillatory activity in the basal ganglia and deep brain stimulation," Movement Disorders, vol. 32, no. 1, pp. 64-69, 2017.

[12] G. Deuschl, J. Raethjen, H. Hellriegel, and R. Elble, "Treatment of patients with essential tremor," The Lancet Neurology, vol. 10, no. 2, pp. 148-161, 2011.

[13] B. Picconi, D. Centonze, K. Håkansson et al., "Loss of bidirectional striatal synaptic plasticity in L-DOPA-induced dyskinesia," Nature Neuroscience, vol. 6, no. 5, pp. 501-506, 2003.
[14] R. Erro, M. Picillo, C. Vitale et al., "Clinical clusters and dopaminergic dysfunction in de-novo Parkinson disease," Parkinsonism and Related Disorders, vol. 28, pp. 137-140, 2016.

[15] R. Chandran and H. Abrahamse, "Identifying plant-based natural medicine against oxidative stress and neurodegenerative disorders," Oxidative Medicine and Cellular Longevity, vol. 2020, Article ID 8648742, 9 pages, 2020.

[16] S. Farah, D. G. Anderson, and R. Langer, "Physical and mechanical properties of PLA, and their functions in widespread applications - A comprehensive review," Advanced Drug Delivery Reviews, vol. 107, pp. 367-392, 2016.

[17] B. Muszyńska, M. Łojewski, J. Rojowski, W. Opoka, and K. Sułkowska-Ziaja, "Natural products of relevance in the prevention and supportive treatment of depression," Psychiatria Polska, vol. 49, no. 3, pp. 435-453, 2015.

[18] Y. J. Zhang, R. Y. Gan, S. Li et al., "Antioxidant phytochemicals for the prevention and treatment of chronic diseases," Molecules, vol. 20, no. 12, pp. 21138-21156, 2015.

[19] T. Isah, "Stress and defense responses in plant secondary metabolites production," Biological Research, vol. 52, no. 1, p. 39, 2019.

[20] M. Naoi, K. Inaba-Hasegawa, M. Shamoto-Nagai, and W. Maruyama, "Neurotrophic function of phytochemicals for neuroprotection in aging and neurodegenerative disorders: modulation of intracellular signaling and gene expression," Journal of Neural Transmission, vol. 124, no. 12, pp. 1515-1527, 2017.

[21] M. A. Hannan, R. Dash, A. A. M. Sohag, M. N. Haque, and I. S. Moon, "Neuroprotection against oxidative stress: phytochemicals targeting TrkB signaling and the Nrf2-ARE antioxidant system," Frontiers in Molecular Neuroscience, vol. 13, 2020.

[22] O. Firuzi, F. Moosavi, R. Hosseini, and L. Saso, "Modulation of neurotrophic signaling pathways by\&nbsp;polyphenols," Drug Design, Development and Therapy, vol. 10, 2015.

[23] R. Venkatesan, E. Ji, and S. Y. Kim, "Phytochemicals that regulate neurodegenerative disease by targeting neurotrophins: a comprehensive review," BioMed Research International, vol. 2015, Article ID 814068, 22 pages, 2015.

[24] C. Tohda, "New age therapy for Alzheimer's disease by neuronal network reconstruction," Biological and Pharmaceutical Bulletin, vol. 39, no. 10, pp. 1569-1575, 2016.

[25] S. Jang and R. W. Johnson, "Can consuming flavonoids restore old microglia to their youthful state?," Nutrition Reviews, vol. 68, no. 12, pp. 719-728, 2010.

[26] Y. Gao, X. Xu, S. Chang et al., "Totarol prevents neuronal injury in vitro and ameliorates brain ischemic stroke: potential roles of Akt activation and HO-1 induction," Toxicology and Applied Pharmacology, vol. 289, no. 2, pp. 142-154, 2015.

[27] S. H. Kwon, S. X. Ma, J. Y. Hwang, S. Y. Lee, and C. G. Jang, "Involvement of the $\mathrm{Nrf} 2 / \mathrm{HO}-1$ signaling pathway in sulfuretin-induced protection against amyloid beta ${ }_{25-35}$ neurotoxicity," Neuroscience, vol. 304, pp. 14-28, 2015.

[28] Y. Zhang, B. Liu, X. Chen et al., "Naringenin ameliorates behavioral dysfunction and neurological deficits in a dgalactose-induced aging mouse model through activation of PI3K/Akt/Nrf2 pathway," Rejuvenation Research, vol. 20, no. 6, pp. 462-472, 2017.

[29] H. Y. Cui, X. J. Zhang, Y. Yang et al., "Rosmarinic acid elicits neuroprotection in ischemic stroke via Nrf2 and heme oxygenase 1 signaling," Neural Regeneration Research, vol. 13, no. 12, pp. 2119-2128, 2018. 
[30] J. Fang, H. Wang, J. Zhou et al., "Baicalin provides neuroprotection in traumatic brain injury mice model through Akt/Nrf2 pathway," Drug Design, Development and Therapy, vol. 12, pp. 2497-2508, 2018.

[31] Y. Hui, T. Chengyong, L. Cheng, H. Haixia, Z. Yuanda, and Y. Weihua, "Resveratrol attenuates the cytotoxicity induced by amyloid- $\beta 1-42$ in PC12 cells by upregulating heme oxygenase-1 via the PI3K/Akt/Nrf2 pathway," Neurochemical Research, vol. 43, no. 2, pp. 297-305, 2018.

[32] D. Prakash and G. Sharma, Phytochemicals of nutraceutical importance, CABI, 2014.

[33] H. Qi and S. Li, "Dose-response meta-analysis on coffee, tea and caffeine consumption with risk of Parkinson's disease," Geriatrics \& gerontology international, vol. 14, no. 2, pp. 430-439, 2014.

[34] H. Boeing, A. Bechthold, A. Bub et al., "Critical review: vegetables and fruit in the prevention of chronic diseases," European Journal of Nutrition, vol. 51, no. 6, pp. 637-663, 2012.

[35] M. H. Eskelinen, T. Ngandu, J. Tuomilehto, H. Soininen, and M. Kivipelto, "Midlife coffee and tea drinking and the risk of late-life dementia: a population-based CAIDE study," Journal of Alzheimer's disease, vol. 16, no. 1, pp. 85-91, 2009.

[36] F. C. Lau, B. Shukitt-Hale, and J. A. Joseph, "Nutritional intervention in brain aging," Inflammation in the pathogenesis of chronic diseases, vol. 42, pp. 299-318, 2007.

[37] S. E. Seidl, J. A. Santiago, H. Bilyk, and J. A. Potashkin, “The emerging role of nutrition in Parkinson's disease," Frontiers in aging neuroscience, vol. 6, p. 36, 2014.

[38] A. A. Farooqui and T. Farooqui, Phytochemicals and human health: pharmacological and molecular aspects, Nova Science Publishers, 2011.

[39] G. P. Kumar and F. Khanum, "Neuroprotective potential of phytochemicals," Pharmacognosy Reviews, vol. 6, no. 12, pp. 81-90, 2012.

[40] E. M. Yahia, "The contribution of fruit and vegetable consumption to human health," Fruit and vegetable phytochemicals, pp. 3-51, 2009.

[41] Z. Y. Wang, J. Y. Liu, C. B. Yang et al., "Neuroprotective natural products for the treatment of Parkinson's disease by targeting the autophagy-lysosome pathway: a systematic review," Phytotherapy Research, vol. 31, no. 8, pp. 11191127, 2017.

[42] H. Zhang, L. Bai, J. He et al., "Recent advances in discovery and development of natural products as source for anti-Parkinson's disease lead compounds," European Journal of Medicinal Chemistry, vol. 141, pp. 257-272, 2017.

[43] H. Javed, M. F. Nagoor Meeran, S. Azimullah, A. Adem, B. Sadek, and S. K. Ojha, "Plant extracts and phytochemicals targeting $\alpha$-synuclein aggregation in Parkinson's disease models," Frontiers in Pharmacology, vol. 9, 2019.

[44] M. Naoi, Y. Wu, M. Shamoto-Nagai, and W. Maruyama, "Mitochondria in neuroprotection by phytochemicals: bioactive polyphenols modulate mitochondrial apoptosis system, function and structure," International journal of molecular sciences, vol. 20, no. 10, p. 2451, 2019.

[45] S. Mandel and M. B. H. Youdim, "Catechin polyphenols: neurodegeneration and neuroprotection in neurodegenerative diseases," Free Radical Biology and Medicine, vol. 37, no. 3, pp. 304-317, 2004.

[46] F. Limanaqi, F. Biagioni, C. L. Busceti et al., "Phytochemicals bridging autophagy induction and alpha-synuclein degrada- tion in parkinsonism," International Journal of Molecular Sciences, vol. 20, no. 13, p. 3274, 2019.

[47] J. Han, X. Y. Pan, Y. Xu et al., "Curcumin induces autophagy to protect vascular endothelial cell survival from oxidative stress damage," Autophagy, vol. 8, no. 5, pp. 812-825, 2012.

[48] M. Holczer, B. Besze, V. Zámbó, M. Csala, G. Bánhegyi, and O. Kapuy, "Epigallocatechin-3-gallate (EGCG) promotes autophagy-dependent survival via influencing the balance of mTOR-AMPK pathways upon endoplasmic reticulum stress," Oxidative Medicine and Cellular Longevity, vol. 2018, Article ID 6721530, 15 pages, 2018.

[49] B. Uttara, A. Singh, P. Zamboni, and R. Mahajan, "Oxidative stress and neurodegenerative diseases: a review of upstream and downstream antioxidant therapeutic options," Current Neuropharmacology, vol. 7, no. 1, pp. 65-74, 2009.

[50] J. B. Schulz, J. Lindenau, J. Seyfried, and J. Dichgans, "Glutathione, oxidative stress and neurodegeneration," European Journal of Biochemistry, vol. 267, no. 16, pp. 4904-4911, 2000.

[51] S. Takahashi, I. Takahashi, H. Sato, Y. Kubota, S. Yoshida, and Y. Muramatsu, "Age-related changes in the concentrations of major and trace elements in the brain of rats and mice," Biological Trace Element Research, vol. 80, no. 2, pp. 145-158, 2001.

[52] C. J. Maynard, R. Cappai, I. Volitakis et al., "Overexpression of Alzheimer's Disease Amyloid- $\beta$ Opposes the Agedependent Elevations of Brain Copper and Iron*," J. Biol. Chem, vol. 277, no. 47, pp. 44670-44676, 2002.

[53] A. Herrera, P. Muñoz, H. W. M. Steinbusch, and J. SeguraAguilar, "Are dopamine oxidation metabolites involved in the loss of dopaminergic neurons in the nigrostriatal system in Parkinson's disease?," ACS Chemical Neuroscience, vol. 8, no. 4, pp. 702-711, 2017.

[54] G. H. Kim, J. E. Kim, S. J. Rhie, and S. Yoon, "The role of oxidative stress in neurodegenerative diseases," Experimental neurobiology, vol. 24, no. 4, pp. 325-340, 2015.

[55] M. H. Yan, X. Wang, and X. Zhu, "Mitochondrial defects and oxidative stress in Alzheimer disease and Parkinson disease," Free Radical Biology and Medicine, vol. 62, pp. 90-101, 2013.

[56] M. Y. Cha, D. K. Kim, and I. Mook-Jung, "The role of mitochondrial DNA mutation on neurodegenerative diseases," Experimental \& molecular medicine, vol. 47, no. 3, p. e150, 2015.

[57] J. Blesa, I. Trigo-Damas, A. Quiroga-Varela, and V. R. Jackson-Lewis, "Oxidative stress and Parkinson's disease," Frontiers in Neuroanatomy, vol. 9, 2015.

[58] H. Kumar, H. W. Lim, S. V. More et al., "The role of free radicals in the aging brain and Parkinson's disease: convergence and parallelism," International Journal of Molecular Sciences, vol. 13, no. 8, pp. 10478-10504, 2012.

[59] A. H. V. Schapira, "Mitochondrial dysfunction in Parkinson's disease," Cell Death and Differentiation, vol. 14, no. 7, pp. 1261-1266, 2007.

[60] H. Büeler, "Impaired mitochondrial dynamics and function in the pathogenesis of Parkinson's disease," Experimental Neurology, vol. 218, no. 2, pp. 235-246, 2009.

[61] R. Perfeito, M. Ribeiro, and A. C. Rego, "Alpha-synucleininduced oxidative stress correlates with altered superoxide dismutase and glutathione synthesis in human neuroblastoma SH-SY5Y cells," Archives of Toxicology, vol. 91, no. 3, pp. 1245-1259, 2017.

[62] T. Yasuhara, K. Hara, K. D. Sethi, J. C. Morgan, and C. V. Borlongan, "Increased 8-OHdG levels in the urine, serum, 
and substantia nigra of hemiparkinsonian rats," Brain Research, vol. 1133, no. 1, pp. 49-52, 2007.

[63] X. Hu, Z. Wang, H. Wu, W. Jiang, and R. Hu, "Ras ssDNA aptamer inhibits vascular smooth muscle cell proliferation and migration through MAPK and PI3K pathways," International Journal of Molecular Medicine, vol. 35, no. 5, pp. 13551361, 2015.

[64] S. Matsuda, Y. Ikeda, M. Murakami, Y. Nakagawa, A. Tsuji, and Y. Kitagishi, "Roles of PI3K/AKT/GSK3 pathway involved in psychiatric illnesses," Diseases, vol. 7, no. 1, p. 22, 2019.

[65] I. Buendia, P. Michalska, E. Navarro, I. Gameiro, J. Egea, and R. León, "Nrf2-ARE pathway: an emerging target against oxidative stress and neuroinflammation in neurodegenerative diseases," Pharmacology and Therapeutics, vol. 157, pp. 84104, 2016.

[66] S. Camandola and M. P. Mattson, "NF- $\kappa$ B as a therapeutic target in neurodegenerative diseases," Expert opinion on therapeutic targets, vol. 11, no. 2, pp. 123-132, 2007.

[67] F. Sivandzade, S. Prasad, A. Bhalerao, and L. Cucullo, "NRF2 and NF- $\kappa$ B interplay in cerebrovascular and neurodegenerative disorders: molecular mechanisms and possible therapeutic approaches," Redox biology, vol. 21, p. 101059, 2019.

[68] H. Dong, R. Li, C. Yu, T. Xu, X. Zhang, and M. Dong, "Paeoniflorin inhibition of 6-hydroxydopamine-induced apoptosis in $\mathrm{PC} 12$ cells via suppressing reactive oxygen speciesmediated PKC $\delta / N F-\kappa B$ pathway," Neuroscience, vol. 285, pp. 70-80, 2015.

[69] N. Kaur, B. Lu, R. K. Monroe, S. M. Ward, and S. W. Halvorsen, "Inducers of oxidative stress block ciliary neurotrophic factor activation of Jak/STAT signaling in neurons," Journal of Neurochemistry, vol. 92, no. 6, pp. 1521-1530, 2005.

[70] S. A. Tooze and G. Schiavo, "Liaisons dangereuses: autophagy, neuronal survival and neurodegeneration," Current Opinion in Neurobiology, vol. 18, no. 5, pp. 504-515, 2008.

[71] N. Mizushima, "Autophagy in protein and organelle turnover," Cold Spring Harbor Symposia on Quantitative Biology, vol. 76, pp. 397-402, 2011.

[72] L. Yu, Y. Chen, and S. A. Tooze, "Autophagy pathway: cellular and molecular mechanisms," Autophagy, vol. 14, no. 2, pp. 207-215, 2018.

[73] H. Harris and D. C. Rubinsztein, "Control of autophagy as a therapy for neurodegenerative disease," Nature Reviews Neurology, vol. 8, no. 2, pp. 108-117, 2012.

[74] N. Chen and V. Karantza, "Autophagy as a therapeutic target in cancer," Cancer Biology and Therapy, vol. 11, no. 2, pp. 157-168, 2011.

[75] B. Levine, N. Mizushima, and H. W. Virgin, "Autophagy in immunity and inflammation," Nature, vol. 469, no. 7330, pp. 323-335, 2011.

[76] H. Dong and M. J. Czaja, "Regulation of lipid droplets by autophagy," Trends in Endocrinology and Metabolism, vol. 22, no. 6, pp. 234-240, 2011.

[77] L. Li, X. Zhang, and W. le, "Autophagy dysfunction in Alzheimer's disease," Neurodegenerative Diseases, vol. 4, pp. 265271, 2010.

[78] M. Komatsu, S. Waguri, T. Chiba et al., "Loss of autophagy in the central nervous system causes neurodegeneration in mice," Nature, vol. 441, no. 7095, pp. 880-884, 2006.

[79] T. Hara, K. Nakamura, M. Matsui et al., "Suppression of basal autophagy in neural cells causes neurodegenerative disease in mice," Nature, vol. 441, no. 7095, pp. 885-889, 2006.
[80] R. Deumens, A. Blokland, and J. Prickaerts, "Modeling Parkinson's Disease in Rats: An Evaluation of 6-OHDA Lesions of the Nigrostriatal Pathway," Experimental Neurology, vol. 175, no. 2, pp. 303-317, 2002.

[81] S. Guo, E. Bezard, and B. Zhao, "Protective effect of green tea polyphenols on the SH-SY5Y cells against 6-OHDA induced apoptosis through ROS-NO pathway," Free Radic. Biol. Med, vol. 39, no. 5, pp. 682-695, 2005.

[82] L. Zhou and Y. Cheng, "Alpha-lipoic acid alleviated 6OHDA-induced cell damage by inhibiting AMPK/mTOR mediated autophagy," Neuropharmacology, vol. 155, pp. 98103, 2019.

[83] X. He, W. Yuan, Z. Li, Y. Hou, F. Liu, and J. Feng, "6-Hydroxydopamine induces autophagic flux dysfunction by impairing transcription factor EB activation and lysosomal function in dopaminergic neurons and SH-SY5Y cells," Toxicology Letters, vol. 283, pp. 58-68, 2018.

[84] X. Zhang, X. Cheng, L. Yu et al., "MCOLN1 is a ROS sensor in lysosomes that regulates autophagy," Nature Communications, vol. 7, no. 1, 2016.

[85] L. Li, J. Tan, Y. Miao, P. Lei, and Q. Zhang, "ROS and autophagy: interactions and molecular regulatory mechanisms," Cellular and Molecular Neurobiology, vol. 35, no. 5, pp. 615-621, 2015.

[86] K. Liu, X. Sun, W. Chen, and Y. Sun, "Autophagy: a doubleedged sword for neuronal survival after cerebral ischemia," Neural Regeneration Research, vol. 9, no. 12, p. 1210, 2014.

[87] M. K. McCoy and M. R. Cookson, "DJ-1 regulation of mitochondrial function and autophagy through oxidative stress," Autophagy, vol. 7, no. 5, pp. 531-532, 2011.

[88] G. Krebiehl, S. Ruckerbauer, L. F. Burbulla et al., "Reduced basal autophagy and impaired mitochondrial dynamics due to loss of Parkinson's disease-associated protein DJ-1," PLoS One, vol. 5, no. 2, article e9367, 2010.

[89] M. Meulener, A. J. Whitworth, C. E. Armstrong-Gold et al., "Drosophila DJ-1 Mutants Are Selectively Sensitive to Environmental Toxins Associated with Parkinson 's Disease," Curr. Biol, vol. 15, no. 17, pp. 1572-1577, 2005.

[90] R. H. Kim, P. D. Smith, H. Aleyasin et al., "Hypersensitivity of DJ-1-deficient mice to 1-methyl-4-phenyl-1,2,3,6-tetrahydropyrindine (MPTP) and oxidative stress," Proceedings of the National Academy of Sciences of the United States of America, vol. 102, no. 14, pp. 5215-5220, 2005.

[91] M. Inden, T. Taira, Y. Kitamura et al., "PARK7 DJ-1 protects against degeneration of nigral dopaminergic neurons in Parkinson's disease rat model," Neurobiology of Disease, vol. 24, no. 1, pp. 144-158, 2006.

[92] W. Zhou and C. R. Freed, "DJ-1 Up-regulates Glutathione Synthesis during Oxidative Stress and Inhibits A53T $\alpha$-Synuclein Toxicity," Journal of Biological Chemistry, vol. 280, no. 52, pp. 43150-43158, 2005.

[93] J. C. Paterna, A. Leng, E. Weber, J. Feldon, and H. Büeler, "DJ-1 and parkin modulate dopamine-dependent behavior and inhibit MPTP-induced nigral dopamine neuron loss in mice," Molecular Therapy, vol. 15, no. 4, pp. 698-704, 2007.

[94] M. Inden, Y. Kitamura, K. Takahashi et al., "Protection against dopaminergic neurodegeneration in Parkinson's disease-model animals by a modulator of the oxidized form of DJ-1, a wild-type of familial Parkinson's disease-linked PARK7," Journal of Pharmacological Sciences, vol. 117, no. 3, pp. 189-203, 2011. 
[95] J. N. Guzman, J. Sanchez-Padilla, D. Wokosin et al., "Oxidant stress evoked by pacemaking in dopaminergic neurons is attenuated by DJ-1," Nature, vol. 468, no. 7324, pp. 696700, 2010.

[96] A. T. Dinkova-Kostova and A. Y. Abramov, "The emerging role of Nrf2 in mitochondrial function," Free Radical Biology and Medicine, vol. 88, Part B, pp. 179-188, 2015.

[97] N. K. Zenkov, E. B. Menshchikova, and V. O. Tkachev, "Keap1/Nrf2/ARE redox-sensitive signaling system as a pharmacological target," Biochemistry, vol. 78, no. 1, pp. 19-36, 2013.

[98] A. Sarrafchi, M. Bahmani, H. Shirzad, and M. RafieianKopaei, “Oxidative stress and Parkinson's disease: new hopes in treatment with herbal antioxidants," Current Pharmaceutical Design, vol. 22, no. 2, pp. 238-246, 2015.

[99] X. Pan, D. Yan, D. Wang et al., "Mitochondrion-mediated apoptosis induced by acrylamide is regulated by a balance between Nrf2 antioxidant and MAPK signaling pathways in PC12 cells," Molecular Neurobiology, vol. 54, no. 6, pp. 4781-4794, 2017.

[100] B. L. Ya, Q. Liu, H.-f. Li et al., "Uric acid protects against focal cerebral ischemia/reperfusion-induced oxidative stress via activating Nrf2 and regulating neurotrophic factor expression," Oxidative Medicine and Cellular Longevity, vol. 2018, 10 pages, 2018.

[101] T. Niu, R. Xuan, L. Jiang et al., "Astaxanthin induces the Nrf2/HO-1 antioxidant pathway in human umbilical vein endothelial cells by generating trace amounts of ROS," Journal of Agricultural and Food Chemistry, vol. 66, no. 6, pp. 1551-1559, 2018.

[102] Y. Zhou, S. Duan, Y. Zhou et al., "Sulfiredoxin-1 attenuates oxidative stress via Nrf2/ARE pathway and 2-Cys Prdxs after oxygen-glucose deprivation in astrocytes," Journal of Molecular Neuroscience, vol. 55, no. 4, pp. 941-950, 2015.

[103] A. Cuadrado, P. Moreno-Murciano, and J. Pedraza-Chaverri, "The transcription factor Nrf2 as a new therapeutic target in Parkinson's disease," Expert Opinion on Therapeutic Targets, vol. 13, no. 3, pp. 319-329, 2009.

[104] Q. Wang, W. X. Li, S. X. Dai et al., "Meta-analysis of Parkinson's disease and Alzheimer's disease revealed commonly impaired pathways and dysregulation of NRF2-dependent genes," Journal of Alzheimer's Disease, vol. 56, no. 4, pp. 1525-1539, 2017.

[105] H. Hara, M. Ohta, and T. Adachi, “Apomorphine protects against 6-hydroxydopamine-induced neuronal cell death through activation of the Nrf2-ARE pathway," Journal of neuroscience research, vol. 84, no. 4, pp. 860-866, 2006.

[106] R. J. Jakel, J. A. Townsend, A. D. Kraft, and J. A. Johnson, "Nrf2-mediated protection against 6-hydroxydopamine," Brain Research, vol. 1144, pp. 192-201, 2007.

[107] P. C. Chen, M. R. Vargas, A. K. Pani et al., "Nrf2-mediated neuroprotection in the MPTP mouse model of Parkinson's disease: critical role for the astrocyte," Proceedings of the National Academy of Sciences of the United States of America, vol. 106, no. 8, pp. 2933-2938, 2009.

[108] K. Nakaso, C. Nakamura, H. Sato, K. Imamura, T. Takeshima, and K. Nakashima, "Novel cytoprotective mechanism of anti-parkinsonian drug deprenyl: PI3K and Nrf2-derived induction of antioxidative proteins," Biochemical and Biophysical Research Communications, vol. 339, no. 3, pp. 915-922, 2006.
[109] C. J. Wruck, M. Claussen, G. Fuhrmann et al., "Luteolin protects rat PC 12 and C6 cells against MPP+ induced toxicity via an ERK dependent Keapl-Nrf2-ARE pathway," Journal of Neural Transmission, pp. 57-67, 2007.

[110] H. Y. Li, S. Y. Wu, and N. Shi, "Transcription factor Nrf2 activation by deltamethrin in PC12 cells: involvement of ROS," Toxicology Letters, vol. 171, no. 1-2, pp. 87-98, 2007.

[111] T. T. Huang, D. L. Hao, B. N. Wu, L. L. Mao, and J. Zhang, "Uric acid demonstrates neuroprotective effect on Parkinson's disease mice through Nrf2-ARE signaling pathway," Biochemical and Biophysical Research Communications, vol. 493, no. 4, pp. 1443-1449, 2017.

[112] B. Bao, M. Q. Zhang, Z. Y. Chen et al., "Sulforaphane prevents PC12 cells from oxidative damage via the Nrf2 pathway," Molecular Medicine Reports, vol. 19, no. 6, pp. 48904896, 2019.

[113] R. Li, T. Liang, L. Xu, N. Zheng, K. Zhang, and X. Duan, "Puerarin attenuates neuronal degeneration in the substantia nigra of 6-OHDA- lesioned rats through regulating BDNF expression and activating the Nrf2/ARE signaling pathway," Brain Research, vol. 1523, pp. 1-9, 2013.

[114] C. Li, B. Tang, Y. Feng et al., "Pinostrobin exerts neuroprotective actions in neurotoxin-induced Parkinson's disease models through Nrf2 induction," Journal of agricultural and food chemistry, vol. 66, no. 31, pp. 8307-8318, 2018.

[115] C. Ramassamy, "Emerging role of polyphenolic compounds in the treatment of neurodegenerative diseases: a review of their intracellular targets," European Journal of Pharmacology, vol. 545, no. 1, pp. 51-64, 2006.

[116] S. Das, L. Stark, I. F. Musgrave, T. Pukala, and S. D. Smid, "Bioactive polyphenol interactions with $\beta$ amyloid: a comparison of binding modelling, effects on fibril and aggregate formation and neuroprotective capacity," Food \& Function, vol. 7, no. 2, pp. 1138-1146, 2016.

[117] A. N. Panche, A. D. Diwan, and S. R. Chandra, "Flavonoids: an overview," Journal of Nutritional Science, vol. 5, 2016.

[118] V. Dias, E. Junn, and M. M. Mouradian, "The role of oxidative stress in Parkinson's disease," Journal of Parkinson's Disease, vol. 3, no. 4, pp. 461-491, 2013.

[119] V. Shukla, S. K. Mishra, and H. C. Pant, "Oxidative stress in neurodegeneration," Advances in Pharmacological Sciences, vol. 2011, 13 pages, 2011.

[120] P. Pospíšil, A. Prasad, and M. Rác, "Mechanism of the formation of electronically excited species by oxidative metabolic processes: role of reactive oxygen species," Biomolecules, vol. 9, no. 7, p. 258, 2019.

[121] L. Yi, S. Ma, and D. Ren, "Phytochemistry and bioactivity of citrus flavonoids: a focus on antioxidant, anti-inflammatory, anticancer and cardiovascular protection activities," Phytochemistry Reviews, vol. 16, no. 3, pp. 479-511, 2017.

[122] K. O. Chu, S. O. Chan, C. P. Pang, and C. C. Wang, "Pro-oxidative and antioxidative controls and signaling modification of polyphenolic phytochemicals: contribution to health promotion and disease prevention?," Journal of Agricultural and Food Chemistry, vol. 62, no. 18, pp. 4026-4038, 2014.

[123] M. Naoi, M. Shamoto-Nagai, and W. Maruyama, "Neuroprotection of multifunctional phytochemicals as novel therapeutic strategy for neurodegenerative disorders: antiapoptotic and antiamyloidogenic activities by modulation of cellular signal pathways," Future Neurology, vol. 14, no. 1, article FNL9, 2019. 
[124] C. Echeverry, F. Arredondo, J. A. Abin-Carriquiry et al., "Pretreatment with natural flavones and neuronal cell survival after oxidative stress: a structure-activity relationship study," Journal of Agricultural and Food Chemistry, vol. 58, no. 4, pp. 2111-2115, 2010.

[125] C. Santos-Buelga and A. Scalbert, "Proanthocyanidins and tannin-like compounds - nature, occurrence, dietary intake and effects on nutrition and health," Journal of the Science of Food and Agriculture, vol. 80, no. 7, pp. 1094-1117, 2000.

[126] Q. Q. Wang, N. Cheng, W. B. Yi, S. M. Peng, and X. Q. Zou, "Synthesis, nitric oxide release, and $\alpha$-glucosidase inhibition of nitric oxide donating apigenin and chrysin derivatives," Bioorganic and Medicinal Chemistry, vol. 22, no. 5, pp. 1515-1521, 2014.

[127] O. M. Ighodaro and O. A. Akinloye, "First line defence antioxidants-superoxide dismutase (SOD), catalase (CAT) and glutathione peroxidase (GPX): their fundamental role in the entire antioxidant defence grid," Alexandria Journal of Medicine, vol. 54, no. 4, pp. 287-293, 2018.

[128] X. Mao, C. Gu, D. Chen, B. Yu, and J. He, "Oxidative stressinduced diseases and tea polyphenols," Oncotarget, vol. 8, no. 46, pp. 81649-81661, 2017.

[129] M. Srinivasan, A. R. Sudheer, and V. P. Menon, "Ferulic acid: therapeutic potential through its antioxidant property," Journal of Clinical Biochemistry and Nutrition, vol. 40, no. 2, pp. 92-100, 2007.

[130] F. Shahidi and P. Ambigaipalan, "Phenolics and polyphenolics in foods, beverages and spices: Antioxidant activity and health effects - A review," Journal of functional foods, vol. 18, pp. 820-897, 2015.

[131] S. M. Poulose, N. Thangthaeng, M. G. Miller, and B. ShukittHale, "Effects of pterostilbene and resveratrol on brain and behavior," Neurochemistry International, vol. 89, pp. 227233, 2015.

[132] T. Farkhondeh, S. Samarghandian, and F. Bafandeh, "The cardiovascular protective effects of chrysin: a narrative review on experimental researches," Cardiovascular \& Hematological Agents in Medicinal Chemistry, vol. 17, no. 1, pp. 17-27, 2019.

[133] K. E. Heim, A. R. Tagliaferro, and D. J. Bobilya, "Flavonoid antioxidants: chemistry, metabolism and structure-activity relationships," Journal of Nutritional Biochemistry, vol. 13, no. 10, pp. 572-584, 2002.

[134] S. Lin, G. Zhang, Y. Liao, J. Pan, and D. Gong, "Dietary flavonoids as xanthine oxidase inhibitors: structure-affinity and structure-activity relationships," Journal of agricultural and food chemistry, vol. 63, no. 35, pp. 7784-7794, 2015.

[135] J. Jang, K. S. Sin, H. P. Kim, and H. Park, "Structure and antiinflammatory activity relationships of wogonin derivatives," Archives of Pharmacal Research, vol. 28, no. 9, p. 1103, 2005.

[136] K. M. Sakthivel and C. Guruvayoorappan, "Amentoflavone inhibits iNOS, COX-2 expression and modulates cytokine profile, NF- $\kappa \mathrm{B}$ signal transduction pathways in rats with ulcerative colitis," International Immunopharmacology, vol. 17, no. 3, pp. 907-916, 2013.

[137] R. Mani and V. Natesan, "Chrysin: sources, beneficial pharmacological activities, and molecular mechanism of action," Phytochemistry, vol. 145, pp. 187-196, 2018.

[138] S. M. Somerset and L. Johannot, "Dietary flavonoid sources in Australian adults," Nutrition and cancer, vol. 60, no. 4, pp. 442-449, 2008.
[139] G. Grosso, U. Stepaniak, R. Topor-Mądry, K. Szafraniec, and A. Pająk, "Estimated dietary intake and major food sources of polyphenols in the Polish arm of the HAPIEE study," Nutrition, vol. 30, no. 11-12, pp. 1398-1403, 2014.

[140] P. Lagiou, E. Samoli, A. Lagiou et al., "Flavonoid classes and risk of peripheral arterial occlusive disease: a case- control study in Greece," European journal of clinical nutrition, vol. 60, no. 2, pp. 214-219, 2006.

[141] A. Tresserra-Rimbau, A. Medina-Remón, J. Pérez-Jiménez et al., "Dietary intake and major food sources of polyphenols in a Spanish population at high cardiovascular risk: the PREDIMED study," Nutrition, Metabolism and Cardiovascular Diseases, vol. 23, no. 10, pp. 953-959, 2013.

[142] R. Zamora-Ros, C. Biessy, J. A. Rothwell et al., "Dietary polyphenol intake and their major food sources in the Mexican Teachers' Cohort," British Journal of Nutrition, vol. 120, no. 3, pp. 353-360, 2018.

[143] S. Jun, S. Shin, and H. Joung, "Estimation of dietary flavonoid intake and major food sources of Korean adults," British Journal of Nutrition, vol. 115, no. 3, pp. 480-489, 2016.

[144] Y. Arai, S. Watanabe, M. Kimira, K. Shimoi, R. Mochizuki, and N. Kinae, "Dietary intakes of flavonols, flavones and isoflavones by Japanese women and the inverse correlation between quercetin intake and plasma LDL cholesterol concentration," The Journal of nutrition, vol. 130, no. 9, pp. 2243-2250, 2000.

[145] N. Otaki, M. Kimira, S. I. Katsumata, M. Uehara, S. Watanabe, and K. Suzuki, "Distribution and major sources of flavonoid intakes in the middle-aged Japanese women," Journal of clinical biochemistry and nutrition, vol. 44, no. 3, pp. 231-238, 2009.

[146] Y. Zhang, Y. Li, C. Cao et al., "Dietary flavonol and flavone intakes and their major food sources in Chinese adults," Nutrition and cancer, vol. 62, no. 8, pp. 1120-1127, 2010.

[147] T. Walle, Y. Otake, J. A. Brubaker, U. K. Walle, and P. V. Halushka, "Disposition and metabolism of the flavonoid chrysin in normal volunteers," British Journal of Clinical Pharmacology, vol. 51, no. 2, pp. 143-146, 2001.

[148] U. K. Walle, A. Galijatovic, and T. Walle, "Transport of the flavonoid chrysin and its conjugated metabolites by the human intestinal cell line Caco-2," Biochemical Pharmacology, vol. 58, no. 3, pp. 431-438, 1999.

[149] P. J. Tobin, P. Beale, L. Noney, S. Liddell, L. P. Rivory, and S. Clarke, "A pilot study on the safety of combining chrysin, a non-absorbable inducer of UGT1A1, and irinotecan (CPT-11) to treat metastatic colorectal cancer," Cancer Chemotherapy and Pharmacology, vol. 57, no. 3, pp. 309-316, 2006.

[150] K. A. Youdim, B. Shukitt-Hale, and J. A. Joseph, "Flavonoids and the brain: interactions at the blood-brain barrier and their physiological effects on the central nervous system," Free Radical Biology and Medicine, vol. 37, no. 11, pp. 1683-1693, 2004.

[151] A. T. R. Goes, C. R. Jesse, M. S. Antunes et al., "Protective role of chrysin on 6-hydroxydopamine-induced neurodegeneration a mouse model of Parkinson's disease: Involvement of neuroinflammation and neurotrophins," Chemico-Biological Interactions, vol. 279, pp. 111-120, 2018.

[152] B. Guo, C. Zheng, W. Cai et al., "Multifunction of chrysin in Parkinson's model: anti-neuronal apoptosis, neuroprotection via activation of MEF2D, and inhibition of monoamine 
oxidase-B," Journal of Agricultural and Food Chemistry, vol. 64, no. 26, pp. 5324-5333, 2016.

[153] A. Krishnamoorthy, M. Sevanan, S. Mani, M. Balu, S. Balaji, and R. P, "Chrysin restores MPTP induced neuroinflammation, oxidative stress and neurotrophic factors in an acute Parkinson's disease mouse model," Neuroscience Letters, vol. 709, p. 134382, 2019.

[154] L. del Fabbro, A. Rossito Goes, C. R. Jesse et al., "Chrysin protects against behavioral, cognitive and neurochemical alterations in a 6-hydroxydopamine model of Parkinson's disease," Neuroscience Letters, vol. 706, pp. 158-163, 2019.

[155] E. H. Aksu, F. M. Kandemir, S. Küçükler, and A. Mahamadu, "Improvement in colistin-induced reproductive damage, apoptosis, and autophagy in testes via reducing oxidative stress by chrysin," Journal of Biochemical and Molecular Toxicology, vol. 32, no. 11, p. e22201, 2018.

[156] M. Y. George, A. Esmat, M. G. Tadros, and E. el-Demerdash, "_In vivo_ cellular and molecular gastroprotective mechanisms of chrysin; Emphasis on oxidative stress, inflammation and angiogenesis," European Journal of Pharmacology, vol. 818, pp. 486-498, 2018.

[157] Y. Temel, S. Kucukler, S. Yıldırım, C. Caglayan, and F. M. Kandemir, "Protective effect of chrysin on cyclophosphamideinduced hepatotoxicity and nephrotoxicity via the inhibition of oxidative stress, inflammation, and apoptosis," NaunynSchmiedeberg's Archives of Pharmacology, vol. 393, no. 3, pp. 325-337, 2020.

[158] L. C. Souza, M. S. Antunes, C. B. Filho et al., "Flavonoid chrysin prevents age-related cognitive decline via attenuation of oxidative stress and modulation of BDNF levels in aged mouse brain," Pharmacology Biochemistry and Behavior, vol. 134, pp. 22-30, 2015.

[159] D. Dobrota, M. Matejovicova, E. G. Kurella, and A. A. Boldyrev, "Na/K-ATPase under oxidative stress: molecular mechanisms of injury," Cellular and Molecular Neurobiology, vol. 19, no. 1, pp. 141-149, 1999.

[160] R. Kavya, R. Saluja, S. Singh, and M. Dikshit, "Nitric oxide synthase regulation and diversity: Implications in Parkinson's disease," Nitric Oxide-Biology and Chemistry, vol. 15, no. 4, pp. 280-294, 2006.

[161] A. Tarafdar and G. Pula, "The role of NADPH oxidases and oxidative stress in neurodegenerative disorders," International Journal of Molecular Sciences, vol. 19, no. 12, p. 3824, 2018.

[162] Z. Zhang, G. Li, S. S. W. Szeto et al., "Examining the neuroprotective effects of protocatechuic acid and chrysin on in vitro and in vivo models of Parkinson disease," Free Radical Biology and Medicine, vol. 84, pp. 331-343, 2015.

[163] M. Zeinali, S. A. Rezaee, and H. Hosseinzadeh, "An overview on immunoregulatory and anti-inflammatory properties of chrysin and flavonoids substances," Biomedicine and Pharmacotherapy, vol. 92, pp. 998-1009, 2017.

[164] A. Sarkaki, Y. Farbood, S. M. T. Mansouri et al., "Chrysin prevents cognitive and hippocampal long-term potentiation deficits and inflammation in rat with cerebral hypoperfusion and reperfusion injury," Life Sciences, vol. 226, pp. 202-209, 2019.

[165] T. B. Sampaio, S. Pinton, J. T. da Rocha, B. M. Gai, and C. W. Nogueira, "Involvement of BDNF/TrkB signaling in the effect of diphenyl diselenide on motor function in a Parkinson's disease rat model," European Journal of Pharmacology, vol. 795, pp. 28-35, 2017.
[166] S. Sarkar, J. Raymick, and S. Imam, "Neuroprotective and therapeutic strategies against Parkinson's disease: recent perspectives," International Journal of Molecular Sciences, vol. 17, no. 6, p. 904, 2016.

[167] X. Yan, D. F. Liu, X. Y. Zhang et al., "Vanillin protects dopaminergic neurons against inflammation-mediated cell death by inhibiting ERK1/2, P38 and the NF- $\kappa$ B signaling pathway," International Journal of Molecular Sciences, vol. 18, no. 2 , p. $389,2017$.

[168] C. Dhanalakshmi, U. Janakiraman, T. Manivasagam et al., "Vanillin attenuated behavioural impairments, neurochemical deficts, oxidative stress and apoptosis against rotenone induced rat model of Parkinson's disease," Neurochemical Research, vol. 41, no. 8, pp. 1899-1910, 2016.

[169] P. C. Chao, H. L. Lee, and M. C. Yin, "Asiatic acid attenuated apoptotic and inflammatory stress in the striatum of MPTPtreated mice," Food Function, vol. 7, no. 4, pp. 1999-2005, 2016.

[170] J. Nataraj, T. Manivasagam, A. Justin Thenmozhi, and M. M. Essa, "Neurotrophic effect of asiatic acid, a triterpene of Centella asiatica against chronic 1-methyl 4-phenyl 1, 2, 3, 6tetrahydropyridine hydrochloride/probenecid mouse model of Parkinson's disease: the role of MAPK, PI3K-Akt-GSK3 $\beta$ and mTOR signalling Pathways," Neurochemical Research, vol. 42, no. 5, pp. 1354-1365, 2017.

[171] E. Anis, M. F. Zafeer, F. Firdaus et al., "Ferulic acid reinstates mitochondrial dynamics through PGC1 $\alpha$ expression modulation in 6-hydroxydopamine lesioned rats," Phytotherapy Research, vol. 34, no. 1, pp. 214-226, 2020.

[172] M. H. Askar, A. M. Hussein, S. F. al-Basiony et al., "Effects of exercise and ferulic acid on alpha synuclein and neuroprotective heat shock protein 70 in an experimental model of parkinsonism disease," CNS \& Neurological Disorders - Drug Targets, vol. 18, no. 2, pp. 156-169, 2019.

[173] X. Li, J. Zhang, H. Rong, X. Zhang, and M. Dong, "Ferulic acid ameliorates MPP+/MPTP-induced oxidative stress via ERK1/2-dependent Nrf2 activation: translational implications for Parkinson disease treatment," Molecular Neurobiology, vol. 57, no. 7, pp. 2981-2995, 2020.

[174] M. T. Ardah, M. M. Merghani, and M. E. Haque, "Thymoquinone prevents neurodegeneration against MPTP in vivo and modulates $\alpha$-synuclein aggregation in vitro," Neurochemistry International, vol. 128, pp. 115-126, 2019.

[175] S. S. Ebrahimi, S. Oryan, E. Izadpanah, and K. Hassanzadeh, "Thymoquinone exerts neuroprotective effect in animal model of Parkinson's disease," Toxicology Letters, vol. 276, pp. 108-114, 2017.

[176] R. Sedaghat, M. Roghani, and M. Khalili, "Neuroprotective effect of thymoquinone, the Nigella sativa bioactive compound, in 6-hydroxydopamine-induced hemi-parkinsonian rat model," Iranian journal of pharmaceutical research, vol. 13, no. 1, p. 227, 2014.

[177] A. Sarkaki, Y. Farbood, M. Dolatshahi, S. M. Mansouri, and A. Khodadadi, "Neuroprotective effects of ellagic acid in a rat model of Parkinson's disease," Acta Medica Iranica, vol. 54, no. 8, pp. 494-502, 2016.

[178] T. Baluchnejadmojarad, N. Rabiee, S. Zabihnejad, and M. Roghani, "Ellagic acid exerts protective effect in intrastriatal 6-hydroxydopamine rat model of Parkinson's disease: Possible involvement of ER $\beta / \mathrm{Nrf} / \mathrm{HO}-1$ signaling," Brain Research, vol. 1662, pp. 23-30, 2017.

[179] R. G. dos Santos Nunes, P. S. Pereira, O. O. Elekofehinti et al., "Possible involvement of transcriptional activation of nuclear 
factor erythroid 2-related factor 2 (Nrf2) in the protective effect of caffeic acid on paraquat-induced oxidative damage in Drosophila melanogaster," Pesticide Biochemistry and Physiology, vol. 157, pp. 161-168, 2019.

[180] Y. Zhang, Q. Wu, L. Zhang et al., "Caffeic acid reduces A53T $\alpha$-synuclein by activating JNK/Bcl-2-mediated autophagy in vitro and improves behaviour and protects dopaminergic neurons in a mouse model of Parkinson 's disease," Pharmacological Research, vol. 150, p. 104538, 2019.

[181] S. J. Tsai, C. Y. Chao, and M. C. Yin, "Preventive and therapeutic effects of caffeic acid against inflammatory injury in striatum of MPTP-treated mice," European Journal of Pharmacology, vol. 670, no. 2-3, pp. 441-447, 2011.

[182] Q. Xu, M. Langley, A. G. Kanthasamy, and M. B. Reddy, "Epigallocatechin gallate has a neurorescue effect in a mouse model of Parkinson disease," Journal of Nutrition, vol. 147, no. 10, pp. 1926-1931, 2017.

[183] T. Zhou, M. Zhu, and Z. Liang, “(-)-Epigallocatechin-3-gallate modulates peripheral immunity in the MPTP-induced mouse model of Parkinson's disease," Molecular Medicine Reports, vol. 17, no. 4, pp. 4883-4888, 2018.

[184] Y. Xu, Y. Zhang, Z. Quan et al., "Epigallocatechin gallate (EGCG) inhibits alpha-synuclein aggregation: a potential agent for Parkinson's disease," Neurochemical Research, vol. 41, no. 10, pp. 2788-2796, 2016.

[185] B. W. Kim, S. Koppula, H. Kumar et al., " $\alpha$-Asarone attenuates microglia-mediated neuroinflammation by inhibiting NF kappa B activation and mitigates MPTP-induced behavioral deficits in a mouse model of Parkinson's disease," Neuropharmacology, vol. 97, pp. 46-57, 2015.

[186] S. Zhang, X. H. Gui, L. P. Huang et al., "Neuroprotective effects of $\beta$-asarone against 6-hydroxy dopamine-induced parkinsonism via JNK/Bcl-2/Beclin-1 pathway," Molecular Neurobiology, vol. 53, no. 1, pp. 83-94, 2016.

[187] B. Ning, M. Deng, Q. Zhang, N. Wang, and Y. Fang, “ $\beta$-Asarone inhibits IRE1/XBP1 endoplasmic reticulum stress pathway in 6-OHDA-induced parkinsonian rats," Neurochemical Research, vol. 41, no. 8, pp. 2097-2101, 2016.

[188] A. Anandhan, U. Janakiraman, and T. Manivasagam, “Theaflavin ameliorates behavioral deficits, biochemical indices and monoamine transporters expression against subacute 1methyl-4-phenyl-1,2,3,6-tetrahydropyridine (MPTP)induced mouse model of Parkinson's disease," Neuroscience, vol. 218, pp. 257-267, 2012.

[189] A. Anandhan, K. Tamilselvam, T. Radhiga, S. Rao, M. M. Essa, and T. Manivasagam, "Theaflavin, a black tea polyphenol, protects nigral dopaminergic neurons against chronic MPTP/probenecid induced Parkinson's disease," Brain Research, vol. 1433, pp. 104-113, 2012.

[190] A. Anandhan, M. M. Essa, and T. Manivasagam, "Therapeutic attenuation of neuroinflammation and apoptosis by black tea theaflavin in chronic MPTP/probenecid model of Parkinson's disease," Neurotoxicity Research, vol. 23, no. 2, pp. 166173, 2013.

[191] N. J. Walton, M. J. Mayer, and A. Narbad, "Vanillin," Phytochemistry, vol. 63, no. 5, pp. 505-515, 2003.

[192] S. Vijayalakshmi, X. Disalva, C. Srivastava, and A. Arun, "Vanilla-natural vs artificial: a review," Research Journal of Pharmacy and Technology, vol. 12, no. 6, pp. 3068-3072, 2019.

[193] A. Kundu, "Vanillin biosynthetic pathways in plants," Planta, vol. 245, no. 6, pp. 1069-1078, 2017.
[194] I. S. Kim, D. K. Choi, and H. J. Jung, "Neuroprotective effects of vanillyl alcohol in gastrodia elata blume through suppression of oxidative stress and anti-apoptotic activity in toxininduced dopaminergic MN9D cells," Molecules, vol. 16, no. 7, pp. 5349-5361, 2011.

[195] M. Makni, Y. Chtourou, M. Barkallah, and H. Fetoui, "Protective effect of vanillin against carbon tetrachloride (CCl4)induced oxidative brain injury in rats," Toxicology and Industrial Health, vol. 28, no. 7, pp. 655-662, 2012.

[196] N. M. al-Baqami and R. Z. Hamza, "Synergistic antioxidant capacities of vanillin and chitosan nanoparticles against reactive oxygen species, hepatotoxicity, and genotoxicity induced by aging in male Wistar rats," Human \& Experimental Toxicology, vol. 40, no. 1, pp. 183-202, 2021.

[197] M. Sefi, A. Elwej, M. Chaâbane et al., "Beneficial role of vanillin, a polyphenolic flavoring agent, on maneb-induced oxidative stress, DNA damage, and liver histological changes in Swiss albino mice," Human \& Experimental Toxicology, vol. 38, no. 6, pp. 619-631, 2019.

[198] C. Dhanalakshmi, T. Manivasagam, J. Nataraj, A. Justin Thenmozhi, and M. M. Essa, "Neurosupportive role of vanillin, a natural phenolic compound, on rotenone induced neurotoxicity in SH-SY5Y neuroblastoma cells," Evidence-Based Complementary and Alternative Medicine, vol. 2015, 11 pages, 2015.

[199] B. Nehru, R. Verma, P. Khanna, and S. K. Sharma, "Behavioral alterations in rotenone model of Parkinson's disease: Attenuation by co-treatment of centrophenoxine," Brain Research, vol. 1201, pp. 122-127, 2008.

[200] M. Alam and W. J. Schmidt, "Rotenone destroys dopaminergic neurons and induces parkinsonian symptoms in rats," Behav. Brain Res, vol. 136, no. 1, pp. 317-324, 2002.

[201] K. H. Chang and C. M. Chen, "The role of oxidative stress in Parkinson's disease,” Antioxidants, vol. 9, no. 7, p. 597, 2020.

[202] A. Tai, T. Sawano, F. Yazama, and H. Ito, "Evaluation of antioxidant activity of vanillin by using multiple antioxidant assays," Biochimica et biophysica acta Reviews on cancer, vol. 1810, no. 2, pp. 170-177, 2011.

[203] J. Xu, H. Xu, Y. Liu, H. He, and G. Li, "Vanillin-induced amelioration of depression-like behaviors in rats by modulating monoamine neurotransmitters in the brain," Psychiatry Res, vol. 225, no. 3, pp. 509-514, 2015.

[204] H. Ueno, S. Suemitsu, S. Murakami et al., "Rescue-like behaviour in mice is mediated by their interest in the restraint tool," Sci. Rep, vol. 9, no. 1, p. 10648, 2019.

[205] M. E. Kim, J. Y. Na, Y. D. Park, and J. S. Lee, “Anti-neuroinflammatory effects of vanillin through the regulation of inflammatory factors and NF- $\kappa \mathrm{B}$ signaling in LPSstimulated microglia," Appl. Biochem. Biotechnol, vol. 187, no. 3, pp. 884-893, 2019.

[206] J. T. James and I. Dubery, "Pentacyclic triterpenoids from the medicinal herb, Centella asiatica (L.) Urban," Molecules, vol. 14, no. 10, pp. 3922-3941, 2009.

[207] S. M. Kamble and C. R. Patil, "Asiatic acid ameliorates doxorubicin-induced cardiac and hepato-renal toxicities with Nrf2 transcriptional factor activation in rats," Cardiovascular Toxicology, vol. 18, no. 2, pp. 131-141, 2018.

[208] S. M. Kamble, S. N. Goyal, and C. R. Patil, "Multifunctional pentacyclic triterpenoids as adjuvants in cancer chemotherapy: a review," RSC Advances, vol. 4, no. 63, pp. 3337033382, 2014. 
[209] K. R. Patil, P. Mohapatra, H. M. Patel et al., "Pentacyclic triterpenoids inhibit IKK $\beta$ Mediated activation of nf- $\kappa \mathrm{b}$ pathway: in silico and in vitro evidences," PLoS One, vol. 10, no. 5, article e0125709, 2015.

[210] K. Ternchoocheep, D. Surangkul, and S. Ysothonsreekul, "The recovery and protective effects of asiatic acid on differentiated human neuroblastoma SH-SY5Y cells cytotoxicinduced by cholesterol," Asian Pacific Journal of Tropical Biomedicine, vol. 7, no. 5, pp. 416-420, 2017.

[211] Z. Qi, X. Ci, J. Huang et al., “Asiatic acid enhances Nrf2 signaling to protect HepG2 cells from oxidative damage through Akt and ERK activation," Biomed. Pharmacother, vol. 88, pp. 252-259, 2017.

[212] B. M. F. Gonçalves, J. A. R. Salvador, S. Marín, and M. Cascante, "Synthesis and anticancer activity of novel fluorinated asiatic acid derivatives," European Journal of Medicinal Chemistry, vol. 114, pp. 101-117, 2016.

[213] W. Jiang, M. Li, F. He et al., "Protective effects of asiatic acid against spinal cord injury-induced acute lung injury in rats," Inflammation, vol. 39, no. 6, pp. 1853-1861, 2016.

[214] G. Lingling, Z. Yuan, and L. Weigen, "Preparation, optimization, characterization and in vivo pharmacokinetic study of asiatic acid tromethamine salt-loaded solid lipid nanoparticles," Drug Development and Industrial Pharmacy, vol. 42, no. 8, pp. 1325-1333, 2016.

[215] N. Raval, T. Mistry, N. Acharya, and S. Acharya, "Development of glutathione-conjugated asiatic acid-loaded bovine serum albumin nanoparticles for brain-targeted drug delivery," Journal of Pharmacy and Pharmacology, vol. 67, no. 11, pp. 1503-1511, 2015.

[216] D. Chen, X. Y. Zhang, J. Sun et al., "Asiatic acid protects dopaminergic neurons from neuroinflammation by suppressing mitochondrial ROS production," Biomolecules and Therapeutics, vol. 27, no. 5, pp. 442-449, 2019.

[217] J. Nataraj, T. Manivasagam, A. Justin Thenmozhi, and M. M. Essa, "Neuroprotective effect of asiatic acid on rotenoneinduced mitochondrial dysfunction and oxidative stressmediated apoptosis in differentiated SH-SYS5Y cells," Nutritional Neuroscience, vol. 20, no. 6, pp. 351-359, 2017.

[218] Y. Xiong, H. Ding, M. Xu, and J. Gao, "Protective effects of asiatic acid on rotenone- or $\mathrm{H}_{2} \mathrm{O}_{2}$-induced injury in SHSY5Y cells," Neurochemical Research, vol. 34, no. 4, pp. 746-754, 2009.

[219] M. F. Xu, Y. Y. Xiong, J. K. Liu, J. J. Qian, L. Zhu, and J. Gao, "Asiatic acid, a pentacyclic triterpene in Centella asiatica, attenuates glutamate-induced cognitive deficits in mice and apoptosis in SH-SY5Y cells," Acta Pharmacologica Sinica, vol. 33 , no. 5, pp. 578-587, 2012.

[220] G. J. Huang, C. S. Chiu, H. J. Chen et al., “Antinociceptive activities and the mechanisms of anti-inflammation of asiatic acid in mice," Evidence-Based Complementary and Alternative Medicine, vol. 2011, 10 pages, 2011.

[221] J. U. Welbat, P. Chaisawang, W. Pannangrong, and P. Wigmore, "Neuroprotective properties of asiatic acid against 5-fluorouracil chemotherapy in the hippocampus in an adult rat model," Nutrients, vol. 10, no. 8, p. 1053, 2018.

[222] Z. Zhao and M. H. Moghadasian, "Chemistry, natural sources, dietary intake and pharmacokinetic properties of ferulic acid: a review," Food Chemistry, vol. 109, no. 4, pp. 691-702, 2008.
[223] A. Fardet, "New hypotheses for the health-protective mechanisms of whole-grain cereals: what is beyond fibre?," Nutrition Research Reviews, vol. 23, no. 1, pp. 65-134, 2010.

[224] E. Barone, V. Calabrese, and C. Mancuso, "Ferulic acid and its therapeutic potential as a hormetin for age-related diseases," Biogerontology, vol. 10, no. 2, pp. 97-108, 2009.

[225] S. Ou and K. C. Kwok, "Ferulic acid: pharmaceutical functions, preparation and applications in foods," Journal of the Science of Food and Agriculture, vol. 84, no. 11, pp. 1261$1269,2004$.

[226] M. N. Clifford, "Chlorogenic acids and other cinnamatesnature, occurrence and dietary burden," Journal of the Science of Food and Agriculture, vol. 79, no. 3, pp. 362-372, 1999.

[227] A. Scalbert and G. Williamson, "Dietary intake and bioavailability of polyphenols," The Journal of Nutrition, vol. 130, no. 8, pp. 2073S-2085S, 2000.

[228] W. Wang, Y. Pan, L. Wang et al., "Optimal dietary ferulic acid for suppressing the obesity-related disorders in leptindeficient obese C57BL/6J-ob/ob mice," Journal of Agricultural and Food Chemistry, vol. 67, no. 15, pp. 4250-4258, 2019.

[229] J. Kanski, M. Aksenova, A. Stoyanova, and D. A. Butterfield, "Ferulic acid antioxidant protection against hydroxyl and peroxyl radical oxidation in synaptosomal and neuronal cell culture systems in vitro: structure-activity studies," Journal of Nutritional Biochemistry, vol. 13, no. 5, pp. 273-281, 2002.

[230] N. Khurana and A. Gajbhiye, “Ameliorative effect of _Sida cordifolia_ in rotenone induced oxidative stress model of Parkinson's disease," Neurotoxicology, vol. 39, pp. 57-64, 2013.

[231] C. L. Yu, X. M. Zhao, and Y. C. Niu, "Ferulic acid protects against lead acetate-induced inhibition of neurite outgrowth by upregulating $\mathrm{HO}-1$ in $\mathrm{PC} 12$ cells: involvement of ERK1/2-Nrf2 pathway," Molecular Neurobiology, vol. 53, no. 9, pp. 6489-6500, 2016.

[232] E. Haque, H. Javed, S. Azimullah, S. B. Abul Khair, and S. Ojha, "Neuroprotective potential of ferulic acid in the rotenone model of Parkinson\&rsquo;s disease," Drug Design, Development and Therapy, vol. 9, p. 5499, 2015.

[233] S. Nagarajan, D. R. Chellappan, P. Chinnaswamy, and S. Thulasingam, "Ferulic acid pretreatment mitigates MPTP-induced motor impairment and histopathological alterations in C57Bl/6 mice," Pharmaceutical Biology, vol. 53, no. 11, pp. 1591-1601, 2015.

[234] H. Chen and D. C. Chan, "Mitochondrial dynamics-fusion, fission, movement, and mitophagy-in neurodegenerative diseases," Human Molecular Genetics, vol. 18, no. R2, pp. R169R176, 2009.

[235] Z. Zhang, L. Liu, X. Jiang, S. D. Zhai, and D. Xing, "The essential role of Drp1 and its regulation by S-nitrosylation of parkin in dopaminergic neurodegeneration: implications for Parkinson's disease," Antioxidants \& Redox Signaling, vol. 25, no. 11, pp. 609-622, 2016.

[236] T. F. Outeiro, E. Kontopoulos, S. M. Altmann et al., "Sirtuin 2 inhibitors Rescue -Synuclein-Mediated Toxicity in models of Parkinson's disease," Science, vol. 317, no. 5837, pp. 516-519, 2007.

[237] X. Yang, S. H. Park, H. C. Chang et al., "Sirtuin 2 regulates cellular iron homeostasis via deacetylation of transcription factor NRF2," Journal of Clinical Investigation, vol. 127, no. 4, pp. 1505-1516, 2017. 
[238] S. N. Goyal, C. P. Prajapati, P. R. Gore et al., "Therapeutic potential and pharmaceutical development of thymoquinone: a multitargeted molecule of natural origin," Frontiers in Pharmacology, vol. 8, 2017.

[239] S. Javidi, B. M. Razavi, and H. Hosseinzadeh, "A review of neuropharmacology effects of Nigella sativa and its main component, thymoquinone," Phytotherapy Research, vol. 30, no. 8, pp. 1219-1229, 2016.

[240] A. Tavakkoli, V. Mahdian, B. M. Razavi, and H. Hosseinzadeh, "Review on clinical trials of black seed (Nigella sativa) and its active constituent, thymoquinone," Journal of pharmacopuncture, vol. 20, no. 3, pp. 179-193, 2017.

[241] S. Darakhshan, A. Bidmeshki Pour, A. Hosseinzadeh Colagar, and S. Sisakhtnezhad, "Thymoquinone and its therapeutic potentials," Pharmacological Research, vol. 95-96, pp. 138$158,2015$.

[242] C. C. Woo, A. P. Kumar, G. Sethi, and K. H. B. Tan, "Thymoquinone: potential cure for inflammatory disorders and cancer," Biochemical Pharmacology, vol. 83, no. 4, pp. 443-451, 2012.

[243] Z. Gholamnezhad, S. Havakhah, and M. H. Boskabady, "Preclinical and clinical effects of Nigella sativa and its constituent, thymoquinone: A review," Journal of Ethnopharmacology, vol. 190, pp. 372-386, 2016.

[244] A. H. Alhebshi, A. Odawara, M. Gotoh, and I. Suzuki, "Thymoquinone protects cultured hippocampal and human induced pluripotent stem cells-derived neurons against $\alpha$ synuclein-induced synapse damage," Neuroscience Letters, vol. 570, pp. 126-131, 2014.

[245] K. S. Radad, M. M. Al-Shraim, M. F. Moustafa, and W. D. Rausch, "Neuroprotective role of thymoquinone against 1methyl-4-phenylpyridinium-induced dopaminergic cell death in primary mesencephalic cell culture," Neurosciences, vol. 20, 2015.

[246] K. Radad, R. Moldzio, M. Taha, and W. D. Rausch, "Thymoquinone protects dopaminergic neurons against $\mathrm{MPP}+$ and rotenone," Phytotherapy Research, vol. 23, no. 5, pp. 696700, 2009.

[247] M. K. Cobourne-Duval, E. Taka, P. Mendonca, and K. F. A. Soliman, "Thymoquinone increases the expression of neuroprotective proteins while decreasing the expression of proinflammatory cytokines and the gene expression $\mathrm{NF} \kappa \mathrm{B}$ pathway signaling targets in LPS/IFN $\gamma$-activated BV-2 microglia cells," Journal of Neuroimmunology, vol. 320, pp. 87-97, 2018.

[248] K. I. Priyadarsini, S. M. Khopde, S. S. Kumar, and H. Mohan, "Free radical studies of ellagic acid, a natural phenolic antioxidant," Journal of Agricultural and Food Chemistry, vol. 50, no. 7, pp. 2200-2206, 2002.

[249] I. Bala, V. Bhardwaj, S. Hariharan, and M. N. V. R. Kumar, "Analytical methods for assay of ellagic acid and its solubility studies," Journal of pharmaceutical and biomedical analysis, vol. 40, no. 1, pp. 206-210, 2006.

[250] R. Zamora-Ros, V. Knaze, J. A. Rothwell et al., "Dietary polyphenol intake in Europe: the European Prospective Investigation into Cancer and Nutrition (EPIC) study," European journal of nutrition, vol. 55, no. 4, pp. 1359-1375, 2016.

[251] V. Knaze, R. Zamora-Ros, L. Luján-Barroso et al., "Intake estimation of total and individual flavan-3-ols, proanthocyanidins and theaflavins, their food sources and determinants in the European Prospective Investigation into Cancer and
Nutrition (EPIC) study," British journal of nutrition, vol. 108, no. 6, pp. 1095-1108, 2012.

[252] S. Corbett, J. Daniel, R. Drayton, M. Field, R. Steinhardt, and N. Garrett, "Evaluation of the anti-inflammatory effects of ellagic acid," Journal of PeriAnesthesia Nursing, vol. 25, no. 4, pp. 214-220, 2010.

[253] Q. S. Liu, R. Deng, S. Li et al., "Ellagic acid protects against neuron damage in ischemic stroke through regulating the ratio of Bcl-2/Bax expression," Applied Physiology, Nutrition, and Metabolism, vol. 42, no. 8, pp. 855-860, 2017.

[254] K. L. Khanduja, P. K. Avti, S. Kumar, N. Mittal, K. K. Sohi, and C. M. Pathak, "Anti-apoptotic activity of caffeic acid, ellagic acid and ferulic acid in normal human peripheral blood mononuclear cells: a Bcl-2 independent mechanism," Biochimica et Biophysica Acta, vol. 1760, no. 2, pp. 283-289, 2006.

[255] N. Seeram, L. Adams, S. Henning et al., "In vitro antiproliferative, apoptotic and antioxidant activities of punicalagin, ellagic acid and a total pomegranate tannin extract are enhanced in combination with other polyphenols as found in pomegranate juice," Journal of Nutritional Biochemistry, vol. 16, no. 6, pp. 360-367, 2005.

[256] N. P. Seeram, R. Lee, and D. Heber, "Bioavailability of ellagic acid in human plasma after consumption of ellagitannins from pomegranate (Punica granatum L.) juice," Clinica Chimica Acta, vol. 348, no. 1-2, pp. 63-68, 2004.

[257] B. Cerdá, R. Llorach, J. J. Cerón, J. C. Espín, and F. A. TomásBarberán, "Evaluation of the bioavailability and metabolism in the rat of punicalagin, an antioxidant polyphenol from pomegranate juice," European Journal of Nutrition, vol. 42, no. 1, pp. 18-28, 2003.

[258] F. Lei, D. M. Xing, L. Xiang et al., "Pharmacokinetic study of ellagic acid in rat after oral administration of pomegranate leaf extract," Journal of Chromatography B, Analytical Technologies in the Biomedical and Life Sciences, vol. 796, no. 1, pp. 189-194, 2003.

[259] L. Yan, P. Yin, C. Ma, and Y. Liu, "Method development and validation for pharmacokinetic and tissue distributions of ellagic acid using ultrahigh performance liquid chromatographytandem mass spectrometry (UPLC-MS/MS)," Molecules, vol. 19, no. 11, pp. 18923-18935, 2014.

[260] L. Pari and R. Sivasankari, "Effect of ellagic acid on cyclosporine A-induced oxidative damage in the liver of rats," Fundamental \& Clinical Pharmacology, vol. 22, no. 4, pp. 395-401, 2008.

[261] S. Mehrzadi, I. Fatemi, A. R. Malayeri et al., "Ellagic acid mitigates sodium arsenite-induced renal and hepatic toxicity in male Wistar rats," Pharmacological Reports, vol. 70, no. 4, pp. 712-719, 2018.

[262] L. P. Tancheva, M. I. Lazarova, A. V. Alexandrova et al., "Neuroprotective mechanisms of three natural antioxidants on a rat model of Parkinson's disease: a comparative study," Antioxidants, vol. 9, no. 1, p. 49, 2020.

[263] D. Khatri and A. R. Juvekar, "Kinetics of inhibition of monoamine oxidase using curcumin and ellagic acid," Pharmacognosy Magazine, vol. 12, no. 46, p. 116, 2016.

[264] I. Lastres-Becker, A. J. García-Yagüe, R. H. Scannevin et al., "Repurposing the NRF2 activator dimethyl fumarate as therapy against synucleinopathy in Parkinson's disease," Antioxidants \& Redox Signaling, vol. 25, no. 2, pp. 61-77, 2016. 
[265] Y. Wei, G.-F. Zhu, C.-Q. Zheng et al., "Ellagic acid protects from rotenone-induced dopaminergic neuronal damage $<$ $\mathrm{i}>\mathrm{via}</ \mathrm{i}>$ activation of Nrf2 signaling in astroglia," SSRN Electronic Journal, 2020.

[266] V. Marques and A. Farah, "Chlorogenic acids and related compounds in medicinal plants and infusions," Food Chem, vol. 113, no. 4, pp. 1370-1376, 2009.

[267] F. Aladedunye, Y. Catel, and R. Przybylski, "Novel caffeic acid amide antioxidants: synthesis, radical scavenging activity and performance under storage and frying conditions," Food Chem, vol. 130, no. 4, pp. 945-952, 2012.

[268] Y. Feng, Y. W. Lu, P. H. Xu et al., "Caffeic acid phenethyl ester and its related compounds limit the functional alterations of the isolated mouse brain and liver mitochondria submitted to in vitro anoxia-reoxygenation: relationship to their antioxidant activities," Biochim Biophys Acta, vol. 1780, no. 4, pp. 659-672, 2008.

[269] X. Wang, S. Stavchansky, S. M. Kerwin, and P. D. Bowman, "Structure-activity relationships in the cytoprotective effect of caffeic acid phenethyl ester (CAPE) and fluorinated derivatives: effects on heme oxygenase-1 induction and antioxidant activities," European Journal of Pharmacology, vol. 635, no. 1-3, pp. 16-22, 2010.

[270] C. Manach, A. Scalbert, C. Morand, C. Rémésy, and L. Jiménez, "Polyphenols: food sources and bioavailability," The American journal of clinical nutrition, vol. 79, no. 5, pp. 727-747, 2004.

[271] S. Huang, C. P. Zhang, K. Wang, G. Li, and F. L. Hu, "Recent advances in the chemical composition of propolis," Molecules, vol. 19, no. 12, pp. 19610-19632, 2014.

[272] M. F. Tolba, S. S. Azab, A. E. Khalifa, S. Z. Abdel-Rahman, and A. B. Abdel-Naim, "Caffeic acid phenethyl ester, a promising component of propolis with a plethora of biological activities: a review on its anti-inflammatory, neuroprotective, hepatoprotective, and cardioprotective effects," IUBMB life, vol. 65, no. 8, pp. 699-709, 2013.

[273] Y. Sato, S. Itagaki, T. Kurokawa et al., "In vitro and in vivo antioxidant properties of chlorogenic acid and caffeic acid," International Journal of Pharmaceutics, vol. 403, no. 1-2, pp. 136-138, 2011.

[274] D. Turan, H. Abdik, F. Sahin, and E. Avşar Abdik, "Evaluation of the neuroprotective potential of caffeic acid phenethyl ester in a cellular model of Parkinson's disease," European Journal of Pharmacology, vol. 883, p. 173342, 2020.

[275] C. V. Fontanilla, Z. Ma, X. Wei et al., "Caffeic acid phenethyl ester prevents 1-methyl-4-phenyl-1,2,3,6-tetrahydropyridine-induced neurodegeneration," Neuroscience, vol. 188, pp. 135-141, 2011.

[276] S. A. Zaitone, E. Ahmed, N. M. Elsherbiny et al., "Caffeic acid improves locomotor activity and lessens inflammatory burden in a mouse model of rotenone-induced nigral neurodegeneration: Relevance to Parkinson's disease therapy," Pharmacological Reports, vol. 71, no. 1, pp. 32-41, 2019.

[277] I. Miyazaki, M. Asanuma, Y. Kikkawa et al., "Astrocytederived metallothionein protects dopaminergic neurons from dopamine quinone toxicity," Glia, vol. 59, no. 3, pp. 435-451, 2011.

[278] I. Miyazaki, N. Isooka, K. Wada, R. Kikuoka, Y. Kitamura, and M. Asanuma, "Effects of enteric environmental modification by coffee components on neurodegeneration in rotenone-treated mice," Cells, vol. 8, no. 3, p. 221, 2019.
[279] N. A. Fazili and A. Naeem, "Anti-fibrillation potency of caffeic acid against an antidepressant induced fibrillogenesis of human $\alpha$-synuclein: Implications for Parkinson's disease," Biochimie, vol. 108, pp. 178-185, 2015.

[280] R. Singh, N. Akhtar, and T. M. Haqqi, "Green tea polyphenol epigallocatechi3-gallate: inflammation and arthritis," Life Sciences, vol. 86, no. 25-26, pp. 907-918, 2010.

[281] V. K. Sharma, A. Bhattacharya, A. Kumar, and H. K. Sharma, "Health benefits of tea consumption," Tropical Journal of Pharmaceutical Research, vol. 6, no. 3, 2007.

[282] J. D. Lambert and R. J. Elias, "The antioxidant and prooxidant activities of green tea polyphenols: a role in cancer prevention," Archives of Biochemistry and Biophysics, vol. 501, no. 1, pp. 65-72, 2010.

[283] S. A. Mandel, Y. Avramovich-Tirosh, L. Reznichenko et al., "Multifunctional activities of green tea catechins in neuroprotection," Neurosignals, vol. 14, no. 1-2, pp. 46-60, 2005.

[284] K. Unno, F. Takabayashi, T. Kishido, and N. Oku, "Suppressive effect of green tea catechins on morphologic and functional regression of the brain in aged mice with accelerated senescence (SAMP10)," Experimental Gerontology, vol. 39, no. 7, pp. 1027-1034, 2004.

[285] K. Unno, F. Takabayashi, H. Yoshida et al., "Daily consumption of green tea catechin delays memory regression in aged mice," Biogerontology, vol. 8, no. 2, pp. 89-95, 2007.

[286] S. Schaffer and B. Halliwell, "Do polyphenols enter the brain and does it matter? Some theoretical and practical considerations," Genes and Nutrition, vol. 7, no. 2, pp. 99-109, 2012.

[287] H. S. Chow, Y. Cai, I. A. Hakim et al., "Pharmacokinetics and safety of green tea polyphenols after multiple-dose administration of epigallocatechin gallate and polyphenon $\mathrm{E}$ in healthy individuals," Clinical cancer research, vol. 9, no. 9, pp. 3312-3319, 2003.

[288] Ullmann, Haller, Decourt, Girault, Spitzer, and Weber, "Plasma-kinetic characteristics of purified and isolated green tea catechin epigallocatechin gallate (EGCG) after 10 days repeated dosing in healthy volunteers," International journal for vitamin and nutrition research, vol. 74, no. 4, pp. 269-278, 2004.

[289] S. M. Henning, Y. Niu, Y. Liu et al., "Bioavailability and antioxidant effect of epigallocatechin gallate administered in purified form versus as green tea extract in healthy individuals," The Journal of nutritional biochemistry, vol. 16, no. 10, pp. 610-616, 2005.

[290] EFSA Panel on Food Additives and Nutrient Sources added to Food (ANS), M. Younes, P. Aggett et al., "Scientific opinion on the safety of green tea catechins," EFSA Journal, vol. 16, no. 4, article e05239, 2018.

[291] L. C. Lin, M. N. Wang, T. Y. Tseng, Sung, and T. H. Tsai, "Pharmacokinetics of (-)-epigallocatechin-3-gallate in conscious and freely moving rats and its brain regional distribution," Journal of agricultural and food chemistry, vol. 55, no. 4, pp. 1517-1524, 2007.

[292] K. Unno, M. Pervin, A. Nakagawa et al., "Blood-brain barrier permeability of green tea catechin metabolites and their neuritogenic activity in human neuroblastoma SH-SY5Y cells.," Molecular nutrition \& food research, vol. 61, no. 12, p. 1700294, 2017.

[293] J. Y. Choi, C. S. Park, D. J. Kim et al., "Prevention of Nitric Oxide-Mediated 1-Methyl-4-Phenyl-1,2,3,6-Tetrahydropyridine-Induced Parkinson's Disease in Mice by Tea Phenolic 
Epigallocatechin 3-Gallate," Neurotoxicology, vol. 23, no. 3, pp. 367-374, 2002.

[294] K. Ritchie and S. Lovestone, "The dementias," The Lancet, vol. 360, no. 9347, pp. 1759-1766, 2002.

[295] H. Checkoway, K. Powers, T. Smith-Weller, G. M. Franklin, W. T. Longstreth, and P. D. Swanson, "Parkinson's disease risks associated with cigarette smoking, alcohol consumption, and caffeine intake," American Journal of Epidemiology, vol. 155, no. 8, pp. 732-738, 2002.

[296] S. Kuriyama, A. Hozawa, K. Ohmori et al., "Green tea consumption and cognitive function: a cross-sectional study from the Tsurugaya Project," The American Journal of Clinical Nutrition, vol. 83, no. 2, pp. 355-361, 2006.

[297] G. Hu, S. Bidel, P. Jousilahti, R. Antikainen, and J. Tuomilehto, "Coffee and tea consumption and the risk of Parkinson's disease," Movement Disorders, vol. 22, no. 15, pp. 2242-2248, 2007.

[298] J. L. Barranco Quintana, M. F. Allam, A. S. Del Castillo, and R. F. Navajas, "Parkinson's disease and tea: a quantitative review," Journal of the American College of Nutrition, vol. 28, no. 1, pp. 1-6, 2009.

[299] K. Hayat, H. Iqbal, U. Malik, U. Bilal, and S. Mushtaq, "Tea and its consumption: benefits and risks," Critical reviews in food science and nutrition, vol. 55, no. 7, pp. 939-954, 2015.

[300] M. P. Mattson, "Apoptosis in neurodegenerative disorders," Nature Reviews Molecular Cell Biology, vol. 1, no. 2, pp. 120-130, 2000.

[301] S. M. H. Sadrzadeh and Y. Saffari, "Iron and brain disorders," American journal of clinical pathology, 2004.

[302] H.-C. Tseng, M.-H. Wang, K.-C. Chang et al., "Protective effect of (-)epigallocatechin-3-gallate on rotenone-induced parkinsonism-like symptoms in rats," Neurotoxicity Research, 2020.

[303] Y. Levites, O. Weinreb, G. Maor, M. B. H. Youdim, and S. Mandel, "Green tea polyphenol (-)-epigallocatechin-3-gallate prevents N-methyl-4-phenyl-1,2,3,6-tetrahydropyridineinduced dopaminergic neurodegeneration," Journal of Neurochemistry, vol. 78, no. 5, pp. 1073-1082, 2001.

[304] L. Wang, S. Xu, X. Xu, and P. Chan, “(-)-Epigallocatechin-3gallate protects SH-SY5Y cells against 6-OHDA-induced cell death through stat3 activation," Journal of Alzheimer's Disease, vol. 17, no. 2, pp. 295-304, 2009.

[305] R.-R. Hou, J.-Z. Chen, H. Chen, X.-G. Kang, M.-G. Li, and B.R. Wang, "Neuroprotective effects of (-)-epigallocatechin-3gallate (EGCG) on paraquat-induced apoptosis in PC12 cells," Cell Biology International, vol. 32, no. 1, pp. 22-30, 2008.

[306] J. S. AL-amri, M. M. Hagras, and M. I. Mujallid, "Effect of epigallocatechin-3-gallate on inflammatory mediators release in LPS-induced Parkinson's disease in rats," Indian Journal of Experimental Biology, vol. 51, no. 5, 2013.

[307] K. S. Kang, Y. Wen, N. Yamabe, M. Fukui, S. C. Bishop, and B. T. Zhu, "Dual beneficial effects of (-)-epigallocatechin-3gallate on levodopa methylation and hippocampal neurodegeneration: in vitro and in vivo studies," PLoS One, vol. 5, no. 8, article e11951, 2010.

[308] D. E. Ehrnhoefer, J. Bieschke, A. Boeddrich et al., "EGCG redirects amyloidogenic polypeptides into unstructured, offpathway oligomers," Nature structural \& molecular biology, vol. 15, no. 6, pp. 558-566, 2008.

[309] B. Cheng, H. Gong, H. Xiao, R. B. Petersen, L. Zheng, and K. Huang, "Inhibiting toxic aggregation of amyloidogenic proteins: a therapeutic strategy for protein misfolding diseases," Biochimica et Biophysica Acta-General Subjects, vol. 1830, no. 10, pp. 4860-4871, 2013.

[310] J. Lu, T. Fu, Y. Qian et al., "Distribution of $\alpha$-asarone in brain following three different routes of administration in rats," European Journal of Pharmaceutical Sciences, vol. 63, pp. 63-70, 2014.

[311] H. Bin Wu and Y. Q. Fang, "Pharmacokinetics of $\beta$-asarone in rats," Yaoxue Xuebao, vol. 39, no. 10, pp. 836-838, 2004.

[312] Y. Q. Fang, C. Shi, L. Liu, and R. M. Fang, "Analysis of transformation and excretion of $\beta$-asarone in rabbits with GCMS," European journal of drug metabolism and pharmacokinetics, vol. 37, no. 3, pp. 187-190, 2012.

[313] L. Liu and Y. Q. Fang, "Analysis of the distribution of $\beta$-asarone in rat hippocampus, brainstem, cortex and cerebellum with gas chromatography-mass spectrometry (GC-MS)," Journal of Medicinal Plant Research, vol. 5, no. 9, pp. 17281734, 2011.

[314] M. Gupta, K. Kant, R. Sharma, and A. Kumar, "Evaluation of in silico anti-parkinson potential of $\beta$-asarone," Central Nervous System Agents in Medicinal Chemistry, vol. 18, no. 2, pp. 128-135, 2018.

[315] A. Kuma, M. Hatano, M. Matsui et al., "The role of autophagy during the early neonatal starvation period," Nature, vol. 432, no. 7020, pp. 1032-1036, 2004.

[316] T. Pan, S. Kondo, W. Le, and J. Jankovic, "The role of autophagy-lysosome pathway in neurodegeneration associated with Parkinson's disease," Brain, vol. 131, no. 8, pp. 1969-1978, 2008.

[317] J. R. Jheng, J. Y. Ho, and J. T. Horng, "ER stress, autophagy, and RNA viruses," Frontiers in Microbiology, vol. 5, 2014.

[318] G. Wei, Y.-b. Chen, D.-F. Chen et al., “ $\beta$-Asarone inhibits neuronal apoptosis via the CaMKII/CREB/Bcl-2 signaling pathway in an in vitro model and A $\beta$ PP/PS1 mice," Journal of Alzheimer's Disease, vol. 33, no. 3, pp. 863-880, 2013.

[319] Q.-S. Zhang, Z.-H. Wang, J.-L. Zhang, Y.-L. Duan, G.-F. Li, and D.-L. Zheng, "Beta-asarone protects against MPTPinduced Parkinson's disease via regulating long non-coding RNA MALAT1 and inhibiting $\alpha$-synuclein protein expression," Biomedicine \& Pharmacotherapy, vol. 83, pp. 153159, 2016.

[320] X. Hei, M. Xie, J. Xu, J. Li, and T. Liu, “ $\beta$-Asarone exerts antioxidative effects on $\mathrm{H} 2 \mathrm{O} 2$-stimulated $\mathrm{PC} 12$ cells by activating Nrf2/HO-1 pathway," Neurochemical Research, vol. 45, no. 8, pp. 1953-1961, 2020.

[321] L. Huang, M. Deng, S. Zhang, S. Lu, X. Gui, and Y. Fang, “ $\beta$ Asarone and levodopa coadministration increases striatal levels of dopamine and levodopa and improves behavioral competence in Parkinson's rat by enhancing dopa decarboxylase activity," Biomedicine \& Pharmacotherapy, vol. 94, pp. 666-678, 2017.

[322] L. Huang, M. Deng, Y. He, S. Lu, S. Liu, and Y. Fang, “ $\beta$-Asarone increases MEF2D and TH levels and reduces $\alpha$-synuclein level in 6-OHDA-induced rats via regulating the HSP70/MAPK/MEF2D/Beclin-1 pathway: chaperone-mediated autophagy activation, macroautophagy inhibition and HSP70 upexpression," Behav. Brain Res, vol. 313, pp. 370-379, 2016.

[323] C. Schuh and P. Schieberle, "Characterization of the key aroma compounds in the beverage prepared from Darjeeling black tea: quantitative differences between tea leaves and 
infusion," Journal of Agricultural and Food Chemistry, vol. 54, no. 3, pp. 916-924, 2006.

[324] J. He, L. Xu, L. Yang et al., "Anti-oxidative effects of catechins and theaflavins on glutamate-induced HT22 cell damage," RSC Advances, vol. 9, no. 37, pp. 21418-21428, 2019.

[325] S. Liu, H. Lu, Q. Zhao et al., "Theaflavin derivatives in black tea and catechin derivatives in green tea inhibit HIV-1 entry by targeting gp41," Biochimica et Biophysica Acta - General Subjects, vol. 1723, no. 1-3, pp. 270-281, 2005.

[326] A. Ben Lagha and D. Grenier, "Black tea theaflavins attenuate Porphyromonas gingivalis virulence properties, modulate gingival keratinocyte tight junction integrity and exert antiinflammatory activity," Journal of Periodontal Research, vol. 52, no. 3, pp. 458-470, 2017.

[327] S. Kaur, P. Greaves, D. N. Cooke et al., "Breast cancer prevention by green tea catechins and black tea theaflavins in the C3(1) SV40 T,t antigen transgenic mouse model is accompanied by increased apoptosis and a decrease in oxidative DNA adducts," Journal of Periodontal Research, vol. 55, no. 9, pp. 3378-3385, 2007.

[328] G. Grelle, A. Otto, M. Lorenz, R. F. Frank, E. E. Wanker, and J. Bieschke, "Black tea theaflavins inhibit formation of toxic amyloid- $\beta$ and $\alpha$-synuclein fibrils," Biochemistry, vol. 50, no. 49, pp. 10624-10636, 2011.

[329] T. Aizawa, A. Yamamoto, and T. Ueno, "Effect of oral theaflavin administration on body weight, fat, and muscle in healthy subjects: a randomized pilot study," Bioscience, biotechnology, and biochemistry, vol. 81, no. 2, pp. 311-315, 2017.

[330] A. Vogiatzoglou, A. A. Mulligan, R. N. Luben et al., “Assessment of the dietary intake of total flavan-3-ols, monomeric flavan-3-ols, proanthocyanidins and theaflavins in the European Union," British Journal of Nutrition, vol. 111, no. 8, pp. 1463-1473, 2014.

[331] T. P. J. Mulder, C. J. van Platerink, P. J. Wijnand Schuyl, and J. M. M. van Amelsvoort, "Analysis of theaflavins in biological fluids using liquid chromatography- electrospray mass spectrometry," Journal of Chromatography B Biomedical Sciences and Applications, vol. 760, no. 2, pp. 271-279, 2001.

[332] Z. Luo, Y. Zhao, Y. Wang, X. Yang, and B. Zhao, "Protective effect of theaflavins on neuron against 6-hydroxydopamineinduced apoptosis in SH-SY5Y cells," Journal of Clinical Biochemistry and Nutrition, vol. 50, no. 2, pp. 133-138, 2012.

[333] J. Zhang, S. Cai, J. Li et al., "Neuroprotective effects of theaflavins against oxidative stress-induced apoptosis in PC12 cells," Neurochemical research, vol. 41, no. 12, pp. 3364-3372, 2016.

[334] A. Szuster-Ciesielska, A. Tustanowska-Stachura, M. Slotwinska, H. Marmurowska-Michalowska, and M. Kandefer-Szerszeñ, "In vitro immunoregulatory effects of antidepressants in healthy volunteers," Polish journal of pharmacology, vol. 55, no. 3, pp. 353-362, 2003.

[335] S. F. Fonseca, N. B. Padilha, S. Thurow et al., "Ultrasoundpromoted copper-catalyzed synthesis of bis-arylselanyl chrysin derivatives with boosted antioxidant and anticancer activities," Ultrasonics Sonochemistry, vol. 39, pp. 827-836, 2017.

[336] M. Scipioni, G. Kay, I. Megson, and P. K. T. Lin, "Novel vanillin derivatives: synthesis, anti-oxidant, DNA and cellular protection properties," European Journal of Medicinal Chemistry, vol. 143, pp. 745-754, 2018.

[337] S. R. Kim, K. A. Koo, M. K. Lee et al., “Asiatic acid derivatives enhance cognitive performance partly by improving acetyl- choline synthesis," Journal of pharmacy and pharmacology, vol. 56, no. 10, pp. 1275-1282, 2004.

[338] M. Kikugawa, T. Ida, H. Ihara, and T. Sakamoto, "Ferulic acid and its water-soluble derivatives inhibit nitric oxide production and inducible nitric oxide synthase expression in rat primary astrocytes," Bioscience, biotechnology, and biochemistry, vol. 81, no. 8, pp. 1607-1611, 2017.

[339] Y. Wu, Y. G. Shi, X. L. Zheng et al., "Lipophilic ferulic acid derivatives protect PC12 cells against oxidative damageviamodulating $\beta$-amyloid aggregation and activating $\mathrm{Nrf} 2$ enzymes," Food \& Function, vol. 11, no. 5, pp. 4707-4718, 2020.

[340] W. Fu, H. Wang, X. Ren, H. Yu, Y. Lei, and Q. Chen, "Neuroprotective effect of three caffeic acid derivatives _via_ ameliorate oxidative stress and enhance PKA/CREB signaling pathway," Behavioural Brain Research, vol. 328, pp. 81-86, 2017.

[341] T. Wan, Z. Wang, Y. Luo et al., "FA-97, a new synthetic caffeic acid phenethyl ester derivative, protects against oxidative stress-mediated neuronal cell apoptosis and scopolamineinduced cognitive impairment by activating $\mathrm{Nrf} 2 / \mathrm{HO}-1$ signaling," Oxidative Medicine and Cellular Longevity, vol. 2019, 21 pages, 2019.

[342] R. Giacomeli, M. G. de Gomes, J. B. Reolon, S. E. Haas, L. M. Colomé, and C. R. Jesse, "Chrysin loaded lipid-core nanocapsules ameliorates neurobehavioral alterations induced by $\beta$ amyloid $_{1-42}$ in aged female mice," Behavioural Brain Research, vol. 390, p. 112696, 2020.

[343] Y. Zhang, J. Zhao, O. Afzal et al., "Neuroprotective role of chrysin-loaded poly(lactic-co-glycolic acid) nanoparticle against kindling-induced epilepsy through Nrf2/ARE/HO-1 pathway," Journal of Biochemical and Molecular Toxicology, vol. 35, no. 2, article e22634, 2020.

[344] S. Trombino, R. Cassano, T. Ferrarelli, E. Barone, N. Picci, and C. Mancuso, "_Trans_ -ferulic acid-based solid lipid nanoparticles and their antioxidant effect in rat brain microsomes," Colloids and surfaces, vol. 109, pp. 273-279, 2013.

[345] W. Wu, S. Y. Lee, X. Wu et al., "Neuroprotective ferulic acid (FA)-glycol chitosan (GC) nanoparticles for functional restoration of traumatically injured spinal cord," Biomaterials, vol. 35, no. 7, pp. 2355-2364, 2014.

[346] P. Hassanzadeh, E. Arbabi, F. Atyabi, and R. Dinarvand, "Ferulic acid-loaded nanostructured lipid carriers: a promising nanoformulation against the ischemic neural injuries," Life Sciences, vol. 193, pp. 64-76, 2018.

[347] H. M. Fahmy, M. M. Fathy, R. A. Abd-elbadia, and W. M. Elshemey, "Targeting of thymoquinone-loaded mesoporous silica nanoparticles to different brain areas: in vivo study," Life Sciences, vol. 222, pp. 94-102, 2019.

[348] R. Pangeni, S. Sharma, G. Mustafa, J. Ali, and S. Baboota, "Vitamin e loaded resveratrol nanoemulsion for brain targeting for the treatment of Parkinson's disease by reducing oxidative stress," Nanotechnology, vol. 25, no. 48, p. 485102, 2014.

[349] G. Flora, D. Gupta, and A. Tiwari, "Nanocurcumin: a promising therapeutic advancement over native curcumin," Critical Reviews in Therapeutic Drug Carrier Systems, vol. 30, no. 4, pp. 331-368, 2013.

[350] K. S. Avadhani, J. Manikkath, M. Tiwari et al., "Skin delivery of epigallocatechin-3-gallate (EGCG) and hyaluronic acid loaded nano-transfersomes for antioxidant and anti-aging 
effects in UV radiation induced skin damage," Drug Delivery, vol. 24, no. 1, pp. 61-74, 2017.

[351] X.-Y. Xiao, Y.-X. Zhu, J.-Y. Bu, G.-W. Li, J.-H. Zhou, and S.P. Zhou, "Evaluation of neuroprotective effect of thymoquinone nanoformulation in the rodent cerebral ischemia-reperfusion model," BioMed Research International, vol. 2016, 11 pages, 2016.

[352] M. Alam, S. Zameer, A. K. Najmi, F. J. Ahmad, S. S. Imam, and M. Akhtar, "Thymoquinone loaded solid lipid nanoparticles demonstrated antidepressant-like activity in rats via indoleamine 2, 3-dioxygenase pathway," Drug Research, vol. 70, no. 5, pp. 206-213, 2020.

[353] S. Ramachandran and S. Thangarajan, "Thymoquinone loaded solid lipid nanoparticles counteracts 3-nitropropionic acid induced motor impairments and neuroinflammation in rat model of Huntington's disease," Metabolic Brain Disease, vol. 33, no. 5, pp. 1459-1470, 2018.

[354] M. A. el-Missiry, A. I. Othman, M. A. Amer, M. Sedki, S. M. Ali, and I. M. el-Sherbiny, "Nanoformulated ellagic acid ameliorates pentylenetetrazol-induced experimental epileptic seizures by modulating oxidative stress, inflammatory cytokines and apoptosis in the brains of male mice," Metabolic Brain Disease, vol. 35, no. 2, pp. 385-399, 2020.

[355] J. Ahlawat, R. Neupane, E. Deemer, S. T. Sreenivasan, and M. Narayan, "Chitosan-ellagic acid nanohybrid for mitigating rotenone-induced oxidative stress," ACS Applied Materials \& Interfaces, vol. 12, no. 16, pp. 18964-18977, 2020.

[356] A. Cano, M. Ettcheto, M. Espina et al., "Epigallocatechin-3gallate loaded PEGylated-PLGA nanoparticles: A new antiseizure strategy for temporal lobe epilepsy," Nanomedicine: Nanotechnology, Biology and Medicine, vol. 14, no. 4, pp. 1073-1085, 2018.

[357] L. Pan, J. Zhou, F. Ju, and H. Zhu, "Intranasal delivery of $\alpha$ asarone to the brain with lactoferrin-modified mPEG-PLA nanoparticles prepared by premix membrane emulsification," Drug Delivery and Translational Research, vol. 8, no. 1, pp. 83-96, 2018. 